\title{
Absolute Anabelian Cuspidalizations of Configuration Spaces of Proper Hyperbolic Curves over Finite Fields
}

By

\author{
Yuichiro HosHI*
}

\section{Contents}

$\S 0 . \quad$ Introduction

$\S 1$. Exactness Properties of the Graded Lie Algebras Arising from Families of Curves

§2. Fundamental Groups of Configuration Spaces over Finite Fields

$\S 3$. Isomorphisms That Preserve the Fundamental Groups of Tripods

$\S 4$. The Reconstruction of the Fundamental Group of the Configuration Space

References

\begin{abstract}
In the present paper, we study the cuspidalization problem for fundamental groups of configuration spaces of proper hyperbolic curves over finite fields. The goal of this paper is to show that any Frobenius-preserving isomorphism between the geometrically pro- $l$ fundamental groups of hyperbolic curves induces an isomorphism between the geometrically pro- $l$ fundamental groups of the associated configuration spaces.
\end{abstract}

\footnotetext{
Communicated by S. Mochizuki. Received June 29, 2007. Revised March 5, 2008, November 14, 2008.

2000 Mathematics Subject Classification(s): 14H30.

${ }^{*}$ Research Institute for Mathematical Sciences, Kyoto University, Kyoto 606-8502, Japan.
} 


\section{$\S 0$. Introduction}

In this paper, we study the following problem, which is called the "cuspidalization problem" (cf. [7], Introduction):

Problem 0.1. $\quad$ Let $r$ be a positive integer. Then can one reconstruct the fundamental group

$$
\pi_{1}\left(U_{X_{(r)}}\right)
$$

of the $r$-th configuration space $U_{X_{(r)}}$ of a hyperbolic curve $X$ over a field $K$ (i.e., the open subscheme of the $r$-th product of $X$ over $K$ whose complement consists of the diagonals " $\left\{\left(x_{1}, \cdots, x_{r}\right) \mid x_{i}=x_{j}\right\}$ " [where $\left.\left.i \neq j\right]\right)$ from the fundamental group $\pi_{1}(X)$ of $X$ ?

Let $r$ be a positive integer, $X$ a proper hyperbolic curve over a finite field $K$, and $l$ a prime number that is invertible in $K$. We shall denote by $\Pi_{X_{(r)}}$ (respectively, $\Pi_{X_{(r)}}$ ) the geometrically pro-l fundamental group of the $r$-th configuration space $U_{X_{(r)}}$ of $X$ (respectively, of the fiber product

$X_{(r)} \stackrel{\text { def }}{=} \overbrace{X \times_{K} \cdots \times_{K} X}$ of $r$ copies of $X$ over $K)$, i.e., the quotient of $\pi_{1}\left(U_{X_{(r)}}\right)$ (respectively, $\pi_{1}\left(X_{(r)}\right)$ ) by the closed normal subgroup obtained as the kernel of the natural projection from $\pi_{1}\left(U_{X_{(r)}} \otimes_{K} \bar{K}\right)$ (respectively, $\pi_{1}\left(X_{(r)} \otimes_{K} \bar{K}\right)$ ) to

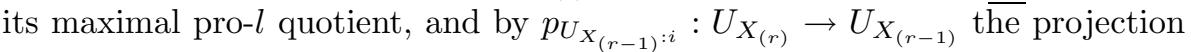
obtained by forgetting the $i$-th factor (where $i=1, \cdots, r$ ). Let $Y$ be a proper hyperbolic curve over a finite field $L$ in which $l$ is invertible; moreover, we shall use similar notations for $Y$. Then the main result of this paper is as follows (cf. Theorem 4.1):

\section{Theorem 0.1. $\quad$ Let}

$$
\alpha_{(1)}: \Pi_{X} \stackrel{\text { def }}{=} \Pi_{X_{(1)}} \stackrel{\sim}{\longrightarrow} \Pi_{Y} \stackrel{\text { def }}{=} \Pi_{Y_{(1)}}
$$

be a Frobenius-preserving isomorphism (cf. Definition 2.5). Then, for any positive integer $r$, there exists a unique isomorphism

$$
\alpha_{(r)}: \Pi_{X_{(r)}} \stackrel{\sim}{\longrightarrow} \Pi_{Y_{(r)}},
$$

well-defined up to composition with a cuspidally inner automorphism (i.e., a $\operatorname{Ker}\left(\Pi_{Y_{(r)}} \rightarrow \Pi_{Y_{(r)}}\right)$-inner automorphism), which is compatible with the natural respective actions of the symmetric group on $r$ letters such that, for $i=$ 
$1, \cdots, r+1$, the following diagram commutes up to composition with a cuspidally inner automorphism (i.e., a $\operatorname{Ker}\left(\Pi_{Y_{(r)}} \rightarrow \Pi_{Y_{(r)}}\right)$-inner automorphism):

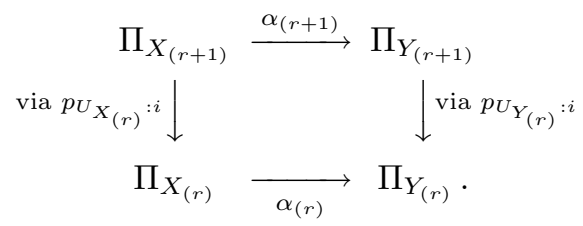

Note that Theorem 0.1 is a generalization of [16], Theorem 3.1. (In [16], Theorem 3.1, the case where $r=2$ is proven.) [16], Theorem 3.1 is used in the proof of Theorem 0.1. Thus, the case where $r \geq 3$ may be regarded as the main new contribution of the present paper.

By Theorem 0.1, we obtain the following result (cf. Corollary 4.1, (i)):

Theorem 0.2. $\quad$ Let

$$
\alpha: \Pi_{X} \stackrel{\sim}{\longrightarrow} \Pi_{Y}
$$

be a Frobenius-preserving isomorphism, $r$ a positive integer, and $\left\{x_{1}, \cdots, x_{r}\right\}$ a set of distinct $K$-rational points of $X$ of cardinality $r$ with an ordering. Then there exist a set $\left\{y_{1}, \cdots, y_{r}\right\}$ of distinct L-rational points of $Y$ of cardinality $r$ with an ordering, and an isomorphism

$$
\alpha^{\text {new }}: \Pi_{X \backslash\left\{x_{1}, \cdots, x_{r}\right\}} \stackrel{\sim}{\longrightarrow} \Pi_{Y \backslash\left\{y_{1}, \cdots, y_{r}\right\}}
$$

of the geometrically pro-l fundamental group of $X \backslash\left\{x_{1}, \cdots, x_{r}\right\}$ with the geometrically pro-l fundamental group of $Y \backslash\left\{y_{1}, \cdots, y_{r}\right\}$ which is compatible with $\alpha$. Moreover, such an isomorphism $\alpha^{\text {new }}$ is uniquely determined up to composition with a cuspidally inner automorphism (i.e., a $\operatorname{Ker}\left(\Pi_{Y \backslash\left\{y_{1}, \cdots, y_{r}\right\}} \rightarrow \Pi_{Y}\right)$ inner automorphism).

An essential part of the proof of the main theorem is to show that the profinite group $\Pi_{X_{(r+1)}}$ can be reconstructed from $\Pi_{X_{(r)}}$ "group-theoretically". This group-theoretic reconstruction of the profinite group $\Pi_{X_{(r+1)}}$ from the given profinite group $\Pi_{X_{(r)}}$ is performed as follows: Let $X_{(r)}^{\log }$ be the $r$-th $\log$ configuration space of $X$ (cf. [7], Definition 1). Then the interior of $X_{(r)}^{\log }$ is naturally isomorphic to the $r$-th configuration space $U_{X_{(r)}}$ of $X$; moreover, it follows from the $\log$ purity theorem that the natural open immersion $U_{X_{(r)}} \hookrightarrow X_{(r)}^{\log }$ induces an isomorphism of the geometrically pro- $l$ fundamental group $\Pi_{X_{(r)}}$ of $U_{X_{(r)}}$ with the geometrically pro- $l \log$ fundamental group of $X_{(r)}^{\log }$. Therefore, to reconstruct $\Pi_{X_{(r+1)}}$, it is enough to reconstruct the geometrically pro- $l$ log 
fundamental group of $X_{(r+1)}^{\log }$. Now it follows from a similar argument to the argument used in the proof of [7], Theorem 4.1, that the images in $\Pi_{X_{(r+1)}}$ of the geometrically pro- $l \log$ fundamental groups of certain irreducible components (equipped with the $\log$ structures induced by the $\log$ structure of $X_{(r+1)}^{\log }$ ) of the divisor at infinity of the $\log$ scheme $X_{(r+1)}^{\log }$ topologically generate the desired profinite group $\Pi_{X_{(r+1)}}$. On the other hand, there exists a topological group $\Pi_{X_{(r+1)}}^{\mathrm{Lie}}$ which arises from the pro-graded Lie algebra obtained by considering the weight filtration of the pro-l fundamental group $\Delta_{X_{(r+1)}}$ of $U_{X_{(r+1)}} \otimes_{K} \bar{K}$ such that the desired profinite group $\Pi_{X_{(r+1)}}$ is naturally embedded in $\Pi_{X_{(r+1}}^{\mathrm{Lie}}$; moreover, this topological group $\Pi_{X_{(r+1)}}^{\mathrm{Lie}}$ can be reconstructed group-theoretically from the given profinite group $\Pi_{X_{(r)}}$ by considering the $G a-$ lois invariant splitting of the subquotients of $\Delta_{X_{(r+1)}}$ with respect to the weight filtration. Note that the fundamental construction of the topological group $\Pi_{X_{(r+1)}}^{\mathrm{Lie}}$ has been initiated, and the fact that the topological group $\Pi_{X_{(r+1)}}^{\mathrm{Lie}}$ can be reconstructed group-theoretically by considering the Galois invariant splitting was first observed by Mochizuki in [16]. Therefore, if one can reconstruct group-theoretically the natural images in $\Pi_{X_{(r+1)}}^{\mathrm{Lie}}$ of the geometrically pro-l $l \log$ fundamental groups of certain irreducible components (equipped with the log structures) of the divisor at infinity of the $\log$ scheme $X_{(r+1)}^{\log }$, then one can construct a subgroup which is isomorphic to the desired profinite group $\Pi_{X_{(r+1)}}$ as the subgroup which is topologically generated by the images reconstructed. This group-theoretic reconstruction of the images of the log fundamental groups of certain irreducible components is performed in Section 4.

\section{Notations and Terminologies:}

\section{Numbers:}

We shall denote by $\mathfrak{P r i m e}$ the set of all prime numbers, by $\mathbb{N}$ the monoid of rational integers $n \geq 0$, by $\mathbb{Z}$ the ring of rational integers, by $\mathbb{Q}$ the field of rational numbers, by $\widehat{\mathbb{Z}}$ (respectively, $\mathbb{Z}_{l}$ ) the profinite completion of $\mathbb{Z}$ (respectively, pro-l completion of $\mathbb{Z}$ for a prime number $l$ ), and by $\mathbb{Q}_{l}$ the field of fractions of $\mathbb{Z}_{l}$.

Let $\Sigma$ be a set of prime numbers, and $n$ an integer. Then we shall say that $n$ is a $\Sigma$-integer if the prime divisors of $n$ are in $\Sigma$. 


\section{Groups:}

Let $G$ be a group, and $H$ a subgroup of $G$. Then we shall write

$$
Z_{G}(H) \stackrel{\text { def }}{=}\{g \in G \mid g \cdot h=h \cdot g \text { for any } h \in H\}
$$

for the center of $H$ in $G$,

$$
N_{G}(H) \stackrel{\text { def }}{=}\left\{g \in G \mid g \cdot H \cdot g^{-1}=H\right\}
$$

for the normalizer of $H$ in $G$, and

$$
C_{G}(H) \stackrel{\text { def }}{=}\left\{g \in G \mid g \cdot H \cdot g^{-1} \cap H \text { has finite index in } g \cdot H \cdot g^{-1} \text { and } H .\right\}
$$

for the commensurator of $H$ in $G$.

Let $G$ be a profinite group and $\Sigma$ a (non-empty) set of prime numbers. Then we shall refer to the quotient

$$
\underset{\lim }{\longleftarrow} / H
$$

of $G$ (where the projective limit is over all open normal subgroups $H \subseteq G$ such that the index $[G: H]$ of $H$ is a $\Sigma$-integer) as the maximal pro- $\Sigma$ quotient of $G$. We shall denote by $G^{(\Sigma)}$ the maximal pro- $\Sigma$ quotient of $G$.

Let $G$ be a topological group. Then we shall denote by $G^{\text {ab }}$ the abelianization of $G$, i.e., the quotient of $G$ by the closed normal subgroup $[G, G]$ generated by the commutators of $G$.

Let $G$ be a Hausdorff topological group. Then we shall denote by $\operatorname{Aut}(G)$ the group of continuous automorphisms, and by $\operatorname{Out}(G)$ the quotient of $\operatorname{Aut}(G)$ by the subgroup $\operatorname{Inn}(G)$ of inner automorphisms of $G$. Note that if $G$ is topologically finitely generated, then by considering a basis of the topology of $G$ consisting of characteristic open subgroups of $G$, we may regard $\operatorname{Aut}(G)$ as being equipped with a topology. This topology on $\operatorname{Aut}(G)$ induces a topology on $\operatorname{Out}(G)$.

Let $G$ be a Hausdorff topological group which is center-free and topologically finitely generated, and $H$ a topological group. Then there exists a natural exact sequence of topological groups:

$$
1 \longrightarrow G \longrightarrow \operatorname{Aut}(G) \longrightarrow \operatorname{Out}(G) \longrightarrow 1
$$

(where $G \rightarrow \operatorname{Aut}(G)$ is defined by letting $G$ act on $G$ by conjugation). For a continuous homomorphism $H \rightarrow \operatorname{Out}(G)$, we shall denote by

$$
G \stackrel{\text { out }}{\rtimes} H
$$


the topological group obtained by pulling back the above exact sequence via the homomorphism $H \rightarrow \operatorname{Out}(G)$, i.e.,

$$
G \stackrel{\text { out }}{\rtimes} H \stackrel{\text { def }}{=} \operatorname{Aut}(G) \times \text { Out }(G) H .
$$

Note that it is immediate that $G \stackrel{\text { out }}{\rtimes} H$ fits into the following natural exact sequence:

$$
1 \longrightarrow G \longrightarrow G \stackrel{\text { out }}{\rtimes} H \longrightarrow H \longrightarrow 1 .
$$

Let $G$ be a profinite group. Then we shall say that $G$ is slim if any open subgroup of $G$ is center-free. Note that it is easily verified that for an exact sequence of profinite groups

$$
1 \longrightarrow G_{1} \longrightarrow G_{2} \longrightarrow G_{3} \longrightarrow 1,
$$

if $G_{1}, G_{3}$ are slim, then $G_{2}$ is slim.

\section{Log schemes:}

A basic reference for the notion of log schemes is [9].

In this paper, $\log$ structures are always considered on the étale sites of schemes.

Let $\mathcal{P}$ be a property of schemes [for example, "quasi-compact", "connected", "normal", "regular"] (respectively, morphisms of schemes [for example, "proper", "finite", "étale", "smooth"]). Then we shall say that a log scheme (respectively, a morphism of $\log$ schemes) satisfies $\mathcal{P}$ if the underlying scheme (respectively, the underlying morphism of schemes) satisfies $\mathcal{P}$.

For a log scheme $X^{\log }$, we shall denote by $X$ (respectively, $\mathcal{M}_{X}$ ) the underlying scheme (respectively, the sheaf of monoids defining the log structure) of $X^{\log }$. For a morphism $f^{\log }$ of $\log$ schemes, we shall denote by $f$ the underlying morphism of schemes.

We shall say that a $\log$ scheme $X^{\log }$ is $f s$ if $X^{\log }$ is integral (i.e., the sheaf $\mathcal{M}_{X}$ is a sheaf of integral monoids), and locally for the étale topology, $X^{\log }$ admits a chart modeled on a finitely generated and saturated monoid.

For fs $\log$ schemes $X^{\log }, Y^{\log }$, and $Z^{\log }$, we shall denote by $X^{\log } \times_{Y^{\log }} Z^{\log }$ the fiber product of $X^{\log }$ and $Z^{\log }$ over $Y^{\log }$ in the category of $f s \log$ schemes. In general, the underlying scheme of $X^{\log } \times_{Y^{\log }} Z^{\log }$ is not naturally isomorphic to $X \times_{Y} Z$. However, since strictness (note that a morphism $f^{\log }: X^{\log } \rightarrow Y^{\log }$ of $\log$ schemes is called strict if the induced morphism on the sheaves of monoids defining the log structures is an isomorphism) is stable under base-change in the 
category of arbitrary $\log$ schemes, if $X^{\log } \rightarrow Y^{\log }$ is strict, then the underlying scheme of $X^{\log } \times_{Y^{\log }} Z^{\log }$ is naturally isomorphic to $X \times_{Y} Z$.

If there exist both schemes and $\log$ schemes in a commutative diagram, then we regard each scheme in the diagram as the log scheme obtained by equipping the scheme with the trivial log structure.

We shall refer to the largest open subset (possibly empty) of the underlying scheme of a $\log$ scheme on which the log structure is trivial as the interior of the log scheme.

Let $X^{\log }$ and $Y^{\log }$ be $\log$ schemes, and $f^{\log }: X^{\log } \rightarrow Y^{\log }$ a morphism of $\log$ schemes. Then we shall refer to the quotient of $\mathcal{M}_{X}$ by the image of the morphism $f^{*} \mathcal{M}_{Y} \rightarrow \mathcal{M}_{X}$ induced by $f^{\log }$ as the relative characteristic sheaf of $f^{\log }$. Moreover, we shall refer to the relative characteristic sheaf of the morphism $X^{\log } \rightarrow X$ induced by the natural inclusion $\mathcal{O}_{X}^{*} \hookrightarrow \mathcal{M}_{X}$ as the characteristic sheaf of $X^{\log }$.

\section{Curves:}

Let $f: X \rightarrow S$ be a morphism of schemes. Then we shall say that $f$ is a family of curves if $f$ is a smooth, geometrically connected morphism whose geometric fibers are one-dimensional. Let $g, r$ be natural numbers. Then we shall say that $f$ is a family of curves of type $(g, r)$ if there exist a family of proper curves $f^{\mathrm{cpt}}: X^{\mathrm{cpt}} \rightarrow S$ (i.e., a family of curves which is a proper morphism) whose geometric fibers are of genus $g$, and a relative divisor $D \subseteq$ $X^{\mathrm{cpt}}$ which is finite étale over $S$ of relative degree $r$ such that $X$ and $X^{\mathrm{cpt}} \backslash D$ are isomorphic over $S$. Moreover, we shall say that $f$ is a family of hyperbolic curves (respectively, tripods) if $f$ is a family of curves of type $(g, r)$ such that $(g, r)$ satisfies $2 g-2+r>0$ (respectively, $(g, r)=(0,3))$. On the other hand, we shall refer to a family of curves (respectively, hyperbolic curves; respectively, tripods) over the spectrum of a field as a curve (respectively, hyperbolic curve; respectively, tripod).

We shall denote by $\overline{\mathcal{M}}_{g, r}$ the moduli stack of $r$-pointed stable curves of genus $g$ whose $r$ sections are equipped with an ordering (cf. [10]), and by $\overline{\mathcal{M}}_{g, r}^{\log }$ the $\log$ stack obtained by equipping $\overline{\mathcal{M}}_{g, r}$ with the log structure associated to the divisor with normal crossings which parametrizes singular curves. Moreover, we shall write $\overline{\mathcal{M}}_{g} \stackrel{\text { def }}{=} \overline{\mathcal{M}}_{g, 0}$ and $\overline{\mathcal{M}}_{g}^{\log } \stackrel{\text { def }}{=} \overline{\mathcal{M}}_{g, 0}^{\log }$.

\section{Fundamental groups:}

For a connected scheme $X$ (respectively, $\log$ scheme $X^{\log }$ ) equipped with a geometric point $\bar{x} \rightarrow X$ (respectively, log geometric point $\widetilde{x}^{\log } \rightarrow X^{\log }$ ), we 
shall denote by $\pi_{1}(X, \bar{x})$ (respectively, $\left.\pi_{1}\left(X^{\log }, \widetilde{x}^{\log }\right)\right)$ the fundamental group of $X$ (respectively, $\log$ fundamental group of $X^{\log }$ ). Since one knows that the fundamental group is determined up to inner automorphisms independently of the choice of basepoint, we shall often omit the basepoint, i.e., we shall often denote by $\pi_{1}(X)$ (respectively, $\pi_{1}\left(X^{\log }\right)$ ) the fundamental group of $X$ (respectively, log fundamental group of $X^{\log }$ ).

For a set $\Sigma$ of prime numbers and a connected scheme $X$ (respectively, log scheme $X^{\log }$ ), we shall refer to the maximal pro- $\Sigma$ quotient of $\pi_{1}(X)$ (respectively, $\pi_{1}\left(X^{\log }\right)$ ) as the pro- $\Sigma$ fundamental group of $X$ (respectively, pro- $\Sigma l o g$ fundamental group of $X^{\log }$ ). Moreover, for a scheme $X$ (respectively, log scheme $X^{\log }$ ) which is geometrically connected and of finite type over a field $K$, we shall refer to the quotient of $\pi_{1}(X)$ (respectively, $\pi_{1}\left(X^{\log }\right)$ ) by the closed normal subgroup obtained as the kernel of the natural projection from $\pi_{1}\left(X \otimes_{K} K^{\text {sep }}\right)$ (respectively, $\pi_{1}\left(X^{\log } \otimes_{K} K^{\text {sep }}\right)$ ) (where $K^{\text {sep }}$ is a separable closure of $K$ ) to its maximal pro- $\Sigma$ quotient $\pi_{1}\left(X \otimes_{K} K^{\text {sep }}\right)^{(\Sigma)}$ (respectively, $\left.\pi_{1}\left(X^{\log \otimes_{K}} K^{\text {sep }}\right)^{(\Sigma)}\right)$ as the geometrically pro- $\Sigma$ fundamental group of $X$ (respectively, geometrically pro- $\Sigma \log$ fundamental group of $\left.X^{\log }\right)$. Thus, the geometrically pro- $\Sigma$ fundamental group $\pi_{1}(X) \frac{(\Sigma)}{\underline{ }}$ of $X$ (respectively, geometrically pro- $\Sigma \log$ fundamental group $\pi_{1}\left(X^{\log }\right) \underline{(\Sigma)}$ of $\left.X^{\log }\right)$ fits into the following exact sequence:

$$
1 \longrightarrow \pi_{1}\left(X \otimes_{K} K^{\mathrm{sep}}\right)^{(\Sigma)} \longrightarrow \pi_{1}(X) \stackrel{(\Sigma)}{\longrightarrow} \operatorname{Gal}\left(K^{\mathrm{sep}} / K\right) \longrightarrow 1
$$

(respectively,

$$
\left.1 \longrightarrow \pi_{1}\left(X^{\log } \otimes_{K} K^{\mathrm{sep}}\right)^{(\Sigma)} \longrightarrow \pi_{1}\left(X^{\log }\right) \stackrel{(\Sigma)}{\longrightarrow} \operatorname{Gal}\left(K^{\mathrm{sep}} / K\right) \longrightarrow 1\right) .
$$

\section{§1. Exactness Properties of the Graded Lie Algebras Arising from Families of Curves}

In this Section, we consider some exactness properties of graded Lie algebras arising from families of curves.

Definition 1.1 (cf. [16], Definition 3.1). Let $l$ be a prime number, $G$, $H$, and $A$ topologically finitely generated pro- $l$ groups, and $\phi: H \rightarrow A$ a (continuous) surjective homomorphism. Suppose further that $A$ is abelian, and that $G$ is an $l$-adic Lie group.

(i) We shall refer to the central filtration

$$
\{H(n)\}(n \geq 1)
$$


of $H$ defined as

$$
\begin{gathered}
H(1) \stackrel{\text { def }}{=} H ; \\
H(2) \stackrel{\text { def }}{=} \operatorname{Ker}(\phi) ; \\
H(m) \stackrel{\text { def }}{=}\left\langle\left[H\left(m_{1}\right), H\left(m_{2}\right)\right] \mid m_{1}+m_{2}=m\right\rangle \text { for } m \geq 3
\end{gathered}
$$

(where $\left\langle N_{i} \mid i \in I\right\rangle$ is the group topologically generated by the $N_{i}$ 's [where $i \in I])$ as the central filtration with respect to the surjection $\phi$.

Let $a, b, n \in \mathbb{Z}$ such that $1 \leq a \leq b, n \geq 1$. Then we shall write

$$
\begin{gathered}
H(a / b) \stackrel{\text { def }}{=} H(a) / H(b) ; \\
\operatorname{Gr}(H)(n) \stackrel{\text { def }}{=} \bigoplus_{m \geq n} H(m / m+1) ; \\
\operatorname{Gr}(H) \stackrel{\text { def }}{=} \operatorname{Gr}(H)(1) ; \\
\operatorname{Gr}(H)(a / b) \stackrel{\text { def }}{=} \operatorname{Gr}(H)(a) / \operatorname{Gr}(H)(b) ; \\
\operatorname{Gr}_{\mathbb{Q}_{l}}(H)(n) \stackrel{\text { def }}{=} \operatorname{Gr}(H)(n) \otimes_{\mathbb{Z}_{l}} \mathbb{Q}_{l} ; \\
\operatorname{Gr}_{\mathbb{Q}_{l}}(H) \stackrel{\text { def }}{=} \operatorname{Gr}(H) \otimes_{\mathbb{Z}_{l}} \mathbb{Q}_{l} ; \\
\operatorname{Gr}_{\mathbb{Q}_{l}}(H)(a / b) \stackrel{\text { def }}{=} \operatorname{Gr}(H)(a / b) \otimes_{\mathbb{Z}_{l}} \mathbb{Q}_{l} ; \\
H(a / \infty) \stackrel{\text { def }}{=} \underset{\leftarrow}{\lim } H(a / b)
\end{gathered}
$$

(where the projective limit is over all integers $b \geq a+1$ ).

(ii) We shall denote by $\operatorname{Lie}(G)$ the Lie algebra over $\mathbb{Q}_{l}$ determined by the $l$ adic Lie group $G$. We shall say that $G$ is nilpotent if there exists a positive integer $m$ such that if we denote by $\{G(n)\}$ the central filtration with respect to the natural surjection $G \rightarrow G^{\text {ab }}$ (cf. (i)), then $G(m)=\{1\}$. If $G$ is nilpotent, then $\operatorname{Lie}(G)$ is a nilpotent Lie algebra over $\mathbb{Q}_{l}$, hence determines a connected, unipotent linear algebraic group $\operatorname{Lin}(G)$, which we shall refer to as the linear algebraic group associated to $G$. In this situation, there is a natural (continuous) homomorphism (with open image)

$$
G \longrightarrow \operatorname{Lin}(G)\left(\mathbb{Q}_{l}\right)
$$

which is determined by the condition that it induces the identity morphism on the associated Lie algebras (cf. [16], Remark 33). In the situation of (i), if $1 \leq a \in \mathbb{Z}$, then we shall write

$$
\operatorname{Lie}(H(a / \infty)) \stackrel{\text { def }}{=} \underset{\longleftarrow}{\lim } \operatorname{Lie}(H(a / b)) ; \operatorname{Lin}(H(a / \infty)) \stackrel{\text { def }}{=} \lim \operatorname{Lin}(H(a / b))
$$


(where the projective limit is over all integers $b \geq a+1$ ). (Note that each $H(a / b)$ is an $l$-adic Lie group.)

Let $K$ be a separably closed field, $l$ a prime number that is invertible in $K$, $S$ a connected locally noetherian normal scheme over $K, g \geq 2$ and $r$ natural numbers, and $f: X \rightarrow S$ a family of hyperbolic curves of type $(g, r)$ (where we refer to the discussion entitled "Curves" in Introduction concerning the term "family of hyperbolic curves of type $(g, r)$ "). We shall denote by

$$
\pi_{1}(X) \underline{(l)}
$$

the geometrically pro- $l$ fundamental group of $X$ (where we refer to the discussion entitled "Fundamental groups" in Introduction concerning the term "geometrically pro-l fundamental group").

Lemma 1.1. Let $\bar{s} \rightarrow S$ be a geometric point of $S$. Then the homomorphism $\pi_{1}(X) \stackrel{(l)}{\rightarrow} \pi_{1}(S)$ induced by $f$ fits into an exact sequence:

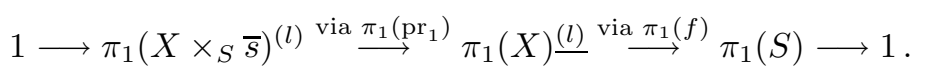

Proof. Let $f^{\mathrm{cpt}}: X^{\mathrm{cpt}} \rightarrow S$ be a (unique, up to canonical isomorphism [cf. the discussion entitled "Curves" in [13], Section 0]) compactification of $f: X \rightarrow S$. If the finite étale covering $D=X^{\text {cpt }} \backslash X \rightarrow S$ is empty or trivial (i.e., $D$ is a disjoint union of copies of $S$, and the covering $D \rightarrow S$ is induced by the identity morphism of $S$ ), then this follows from [23], Proposition 2.3. In general, if $S^{\prime} \rightarrow S$ is a connected finite étale covering of $S$ such that $D \times{ }_{S} S^{\prime} \rightarrow S^{\prime}$ is trivial, then we obtain a commutative diagram

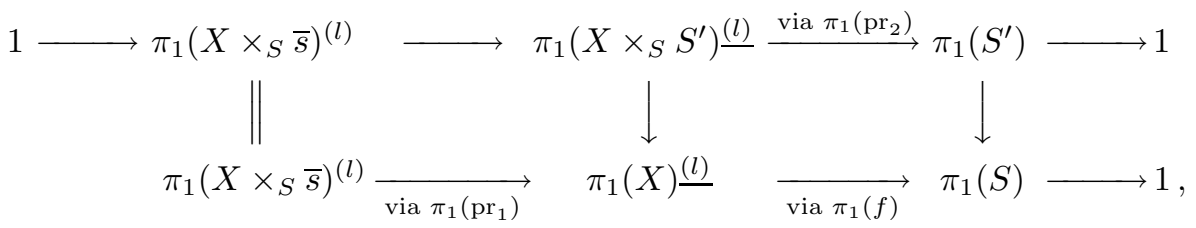

where the horizontal sequences are exact (note that the exactness of the bottom sequence follows from [3], Exposé XIII, Proposition 4.1; Exemples 4.4), and the vertical arrows are injective. Thus, $\pi_{1}\left(X \times_{S} \bar{s}\right)^{(l) \stackrel{\text { via }}{\longrightarrow} \pi_{1}\left(\mathrm{pr}_{1}\right)} \pi_{1}(X) \stackrel{(l)}{\longrightarrow}$ is injective.

We shall denote by

$$
\Delta_{X / S}
$$


the kernel of the homomorphism $\pi_{1}(X) \stackrel{(l)}{\rightarrow} \pi_{1}(S)$ induced by $f$. Then by Lemma 1.1, this pro-l group $\Delta_{X / S}$ is isomorphic to the pro-l fundamental group of a hyperbolic curve of type $(g, r)$ (over a separably closed field). We shall write

$$
\Delta_{X / S}^{\mathrm{cpt}} \stackrel{\text { def }}{=} \Delta_{X^{\mathrm{cpt}} / S},
$$

i.e., the pro-l fundamental group of a geometric fiber of the compactification $f^{\mathrm{cpt}}: X^{\mathrm{cpt}} \rightarrow S$ of $f: X \rightarrow S$. Then we have a natural surjection

$$
\Delta_{X / S} \rightarrow \Delta_{X / S}^{\mathrm{cpt}}
$$

which fits into a commutative diagram

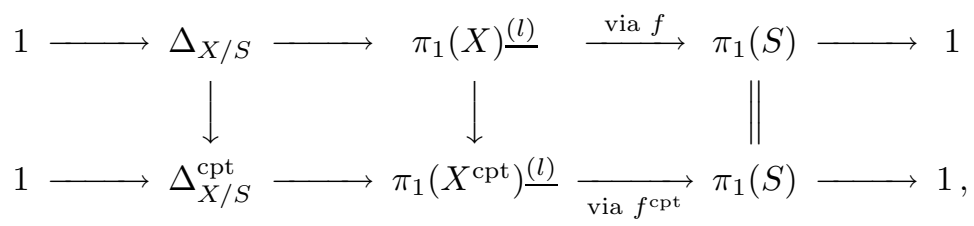

where the horizontal sequences are exact (cf. Lemma 1.1).

We shall denote by

$$
\left\{\Delta_{X / S}(n)\right\}
$$

the central filtration of $\Delta_{X / S}$ with respect to the composite of the natural surjections (cf. Definition 1.1, (i)):

$$
\Delta_{X / S} \rightarrow \Delta_{X / S}^{\mathrm{cpt}} \rightarrow\left(\Delta_{X / S}^{\mathrm{cpt}}\right)^{\mathrm{ab}} .
$$

Remark 1. As is well-known, the graded Lie algebra $\operatorname{Gr}\left(\Delta_{X / S}\right.$ ) (where "Gr" is taken with respect to the central filtration defined above) is center-free (cf. e.g., [2], Theorem 1, (ii), together with [2], Proposition 5).

Now by Lemma 1.1, we obtain an outer representation:

$$
\rho_{X / S}: \pi_{1}(S) \longrightarrow \operatorname{Out}\left(\Delta_{X / S}\right) .
$$

We shall denote by

$$
\operatorname{Out}^{*}\left(\Delta_{X / S}\right) \subseteq \operatorname{Out}\left(\Delta_{X / S}\right)
$$

the subgroup of $\operatorname{Out}\left(\Delta_{X / S}\right)$ whose elements preserve the central filtration $\left\{\Delta_{X / S}(n)\right\}$ of $\Delta_{X / S}$.

Remark 2. If $r \geq 2$, then by the definition of $\operatorname{Out}^{*}\left(\Delta_{X / S}\right)$, we obtain

$$
\operatorname{Out}^{*}\left(\Delta_{X / S}\right) \neq \operatorname{Out}\left(\Delta_{X / S}\right) .
$$


Indeed, this follows immediately from the definition of $\left\{\Delta_{X / S}(n)\right\}$, together with the fact that the assumption that $r \neq 0$ implies that the profinite group $\Delta_{X / S}$ is a free pro-l group.

Proposition 1.1. The outer representation $\rho_{X / S}$ factors through $\operatorname{Out}^{*}\left(\Delta_{X / S}\right)$.

Proof. This follows from the fact that the exact sequence obtained in Lemma 1.1 fits into the commutative diagram in the discussion following Lemma 1.1.

Definition 1.2. We shall say that $f$ is of pro-l-exact type if the sequence

$$
1 \longrightarrow \Delta_{X / S} \longrightarrow \Delta_{X} \stackrel{\text { via } f}{\longrightarrow} \Delta_{S} \longrightarrow 1
$$

naturally induced by the exact sequence obtained in Lemma 1.1 is exact, where $\Delta_{X}$ (respectively, $\left.\Delta_{S}\right)$ is the pro- $l$ fundamental group of $X$ (respectively, $S$ ).

Proposition 1.2. $\quad$ The image of the composite

$$
\pi_{1}(S) \stackrel{\rho_{X / S}}{\longrightarrow} \operatorname{Out}^{*}\left(\Delta_{X / S}\right) \longrightarrow \operatorname{Aut}\left(\left(\Delta_{X / S}^{\mathrm{cpt}}\right)^{\mathrm{ab}}\right)
$$

is a pro-l group (e.g., the action of $\pi_{1}(S)$ on $\left(\Delta_{X / S}^{\mathrm{cpt}}\right)^{\mathrm{ab}}$ is trivial) if and only if $f$ is of pro-l-exact type.

Proof. It is immediate that if $f$ is of pro-l-exact type, then $\rho_{X / S}$ factors through $\Delta_{S}$. Thus, we prove that if the composite in the statement of Proposition 1.2 factors through $\Delta_{S}$, then $f$ is of pro-l-exact type. It follows from [12], Lemma 3.1, (i), that the kernel of the natural morphism

$$
\operatorname{Out}^{*}\left(\Delta_{X / S}\right) \longrightarrow \operatorname{Aut}\left(\left(\Delta_{X / S}^{\mathrm{cpt}}\right)^{\mathrm{ab}}\right)
$$

is a pro-l group. Therefore, the assumption implies that the homomorphism $\rho_{X / S}$ factors through $\Delta_{S}$. Now let us write

$$
\Gamma \stackrel{\text { def }}{=} \Delta_{X / S} \stackrel{\text { out }}{\rtimes} \Delta_{S}
$$

(cf. the discussion entitled "Groups" in Introduction). Then we have a natural morphism $\pi_{1}(X) \stackrel{(l)}{\rightarrow} \Gamma$ that fits into a commutative diagram

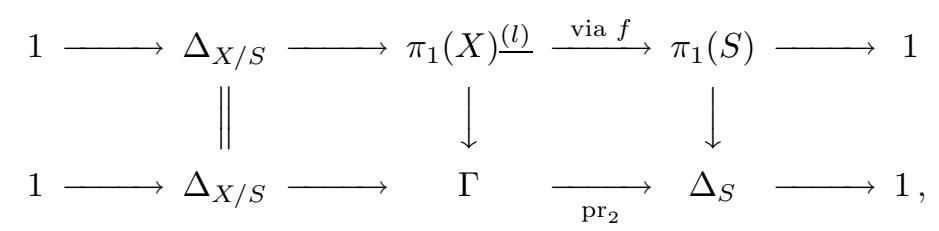


where the horizontal sequences are exact. Note that since $\pi_{1}(S) \rightarrow \Delta_{S}$ is surjective, $\pi_{1}(X) \stackrel{(l)}{\rightarrow} \Gamma$ is also surjective, and that since $\Delta_{X / S}$ and $\Delta_{S}$ are pro- $l, \Gamma$ is also pro- $l$. Now we shall denote by $N_{1}$ (respectively, $N_{2}$ ) the kernel of the natural surjection $\pi_{1}(X) \underline{(l)} \rightarrow \Delta_{X}$ (respectively, $\pi_{1}(X) \underline{(l)} \rightarrow \Gamma$ ). Then the following

(i) $N_{1} \subseteq N_{2}$. (This follows from the fact that $\Gamma$ is pro-l.)

(ii) $\Delta_{X / S} \cap N_{2}=\{1\}$. (This follows from the above diagram.)

(iii) $\Delta_{X / S} \cap N_{1}=\{1\}$. (This follows from (i) and (ii).)

By (ii) and (iii), the following natural sequence is exact

$$
1 \longrightarrow \Delta_{X / S} \longrightarrow \Delta_{X} \longrightarrow \pi_{1}(S) / N_{3} \longrightarrow 1,
$$

where $N_{3}$ is the image of $N_{1}$ via the surjection $\pi_{1}(X) \underline{(l)} \rightarrow \pi_{1}(S)$. Moreover, by (i), this exact sequence fits into a commutative diagram

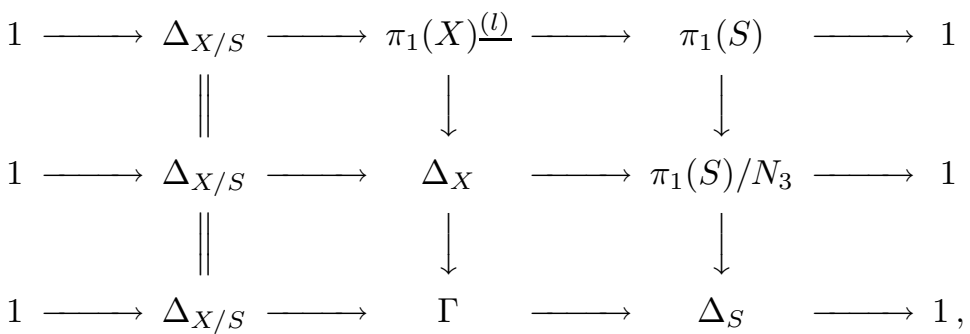

where the horizontal sequences are exact, and all vertical arrows are surjective. Since $\Delta_{X}$ is pro-l, the group $\pi_{1}(S) / N_{3}$ is also pro-l. Thus, the right-hand lower vertical arrow $\pi_{1}(S) / N_{3} \rightarrow \Delta_{S}$, hence also, $\Delta_{X} \rightarrow \Gamma$ is an isomorphism. This completes the proof of Proposition 1.2.

Definition 1.3. Let $A_{X}$ and $A_{S}$ be profinite abelian groups, and $\Delta_{X} \rightarrow$ $A_{X}$ and $\Delta_{S} \rightarrow A_{S}$ (continuous) surjections. Then we shall say that $\left(f, \Delta_{X} \rightarrow\right.$ $A_{X}, \Delta_{S} \rightarrow A_{S}$ ) is of Lie-exact type if the following conditions are satisfied:

(i) $f$ is of pro-l-exact type.

(ii) The surjections $\Delta_{X} \rightarrow A_{X}$ and $\Delta_{S} \rightarrow A_{S}$ fit into a commutative diagram

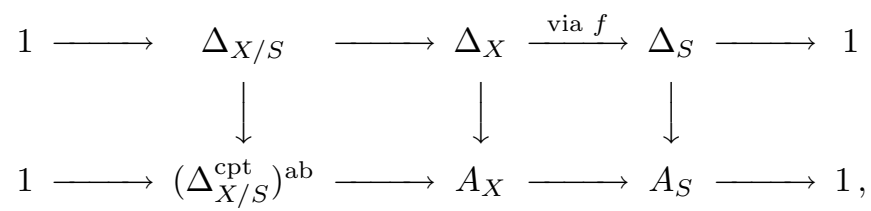

where the bottom sequence is exact. 
(iii) The sequence of graded Lie algebras

$$
1 \longrightarrow \operatorname{Gr}\left(\Delta_{X / S}\right) \longrightarrow \operatorname{Gr}\left(\Delta_{X}\right) \stackrel{\text { via } f}{\longrightarrow} \operatorname{Gr}\left(\Delta_{S}\right) \longrightarrow 1
$$

naturally induced by the exact sequence in Definition 1.2 is exact, where "Gr" of $\operatorname{Gr}\left(\Delta_{X}\right)$ (respectively, $\operatorname{Gr}\left(\Delta_{S}\right)$ ) is taken with respect to the central filtrations

$$
\left\{\Delta_{X}(n)\right\} \text { (respectively, }\left\{\Delta_{S}(n)\right\} \text { ) }
$$

with respect to the surjection $\Delta_{X} \rightarrow A_{X}$ (respectively, $\Delta_{S} \rightarrow A_{S}$ ) [thus, $A_{X} \simeq \Delta_{X}(1 / 2)\left(\right.$ respectively, $\left.\left.A_{S} \simeq \Delta_{S}(1 / 2)\right)\right]$.

Proposition 1.3. Let $A_{X}$ and $A_{S}$ be profinite abelian groups, and $\Delta_{X} \rightarrow A_{X}$ and $\Delta_{S} \rightarrow A_{S}$ surjections. Assume that $\left(f, \Delta_{X} \rightarrow A_{X}, \Delta_{S} \rightarrow A_{S}\right)$ satisfies conditions (i) and (ii) in Definition 1.3. Then the following conditions are equivalent:

(i) ( $\left.f, \Delta_{X} \rightarrow A_{X}, \Delta_{S} \rightarrow A_{S}\right)$ is of Lie-exact type.

(ii) The action of $\Delta_{X}$ on $\Delta_{X / S}(n / n+1)$ and the action of $\Delta_{X}(2)$ on $\Delta_{X / S}(n / n+$ 2) (induced via conjugation) are trivial for any $n \geq 1$.

(ii') The action of $\Delta_{S}$ on $\Delta_{X / S}(n / n+1)$ and the action of $\Delta_{S}(2)$ on $\Delta_{X / S}(n / n+$ 2) (induced via $\rho_{X / S}$ ) are trivial for any $n \geq 1$.

(iii) The action of $\Delta_{X}(m)$ on $\Delta_{X / S}(n / n+m)$ (induced via conjugation) is trivial for any $n, m \geq 1$.

Proof. First, we prove that (i) implies (ii). If (ii) does not hold, then there exists $x \in \Delta_{X / S}(n)$ and $\sigma \in \Delta_{X}(m)$ (where $m=1$ or 2) such that $\sigma \cdot x \cdot \sigma^{-1} \cdot x^{-1} \notin \Delta_{X / S}(n+m)$. On the other hand, by the definition of the filtration $\left\{\Delta_{X}(n)\right\}$, we have that $\sigma \cdot x \cdot \sigma^{-1} \cdot x^{-1} \in \Delta_{X}(n+m) \cap \Delta_{X / S}$. Thus, $\Delta_{X / S}(n+m) \neq \Delta_{X}(n+m) \cap \Delta_{X / S}$. This implies that the natural morphism $\operatorname{Gr}\left(\Delta_{X / S}\right) \rightarrow \operatorname{Gr}\left(\Delta_{X}\right)$ is not injective. Thus, (i) does not hold.

Next, we prove that (ii) implies (iii). This proof will be by induction on $m$. The assertion for $m=1$ and 2 follows from (ii). Assume that $m \geq 3$. Then it follows from the induction hypothesis and a well-known identity due to P. Hall (i.e.,

$$
[A,[B, C]] \subseteq[B,[C, A]] \cdot[C,[A, B]]
$$

for closed normal subgroups $A, B$, and $C$ of an ambient group [cf. e.g., [11], Theorem 5.2]) that

$$
\left[\Delta_{X / S}(n),\left[\Delta_{X}\left(m_{1}\right), \Delta_{X}\left(m_{2}\right)\right]\right] \subseteq \Delta_{X / S}(n+m)
$$


for positive integers $m_{1}$ and $m_{2}$ such that $m_{1}+m_{2}=m$. Thus, since, in general, for a finite set $I$,

$$
\left\langle\left[G, H_{i}\right] \mid i \in I\right\rangle=\left[G,\left\langle H_{i} \mid i \in I\right\rangle\right]
$$

for closed normal subgroups $H_{i}(i \in I)$ of an ambient group $G$, we thus obtain an inclusion

$$
\left[\Delta_{X / S}(n), \Delta_{X}(m)\right] \subseteq \Delta_{X / S}(n+m)
$$

by the definition of the filtration $\left\{\Delta_{X}(n)\right\}$. Therefore, we conclude that (iii) holds.

The assertion that (iii) implies (i) follows from a similar argument to the argument used in the proof of [12], Proposition 3.2 (cf. also Remark 1 and [12], Lemma 3.2).

The equivalence of (ii) and (ii') follows immediately from the exactness of the following sequences:

$$
\begin{gathered}
1 \longrightarrow \Delta_{X / S} \longrightarrow \Delta_{X} \longrightarrow \Delta_{S} \longrightarrow 1 ; \\
1 \longrightarrow \Delta_{X / S}(2) \longrightarrow \Delta_{X}(2) \longrightarrow \Delta_{S}(2) \longrightarrow 1 .
\end{gathered}
$$

Lemma 1.2. $\quad$ Let $I^{\mathrm{cpt}}$ be the kernel of the surjection

$$
\Delta_{X / S} \rightarrow \Delta_{X / S}^{\mathrm{cpt}} .
$$

Let $\bar{s} \rightarrow S$ be a geometric point of $S$. We shall write

$$
D_{\bar{s}} \stackrel{\text { def }}{=} D \times_{S} \bar{s}
$$

where $D \subseteq X^{\mathrm{cpt}}$ is the reduced relative divisor over $S$ obtained as the complement of $X$ in $X^{\mathrm{cpt}}$. Then the following hold:

(i) The submodule

$$
\left(\Delta_{X / S}^{\mathrm{cpt}}\right)^{\mathrm{ab}}=\Delta_{X / S}(1 / 2) \subseteq \operatorname{Gr}\left(\Delta_{X / S}\right)
$$

and the submodule

$$
I^{\mathrm{cpt}} /\left(\Delta_{X / S}(3) \cap I^{\mathrm{cpt}}\right) \subseteq \Delta_{X / S}(2 / 3) \subseteq \operatorname{Gr}\left(\Delta_{X / S}\right)
$$

generate the graded Lie algebra $\operatorname{Gr}\left(\Delta_{X / S}\right)$ (as a Lie algebra). In particular, if $f$ is of pro-l-exact type, then the following conditions are equivalent: 
(1) The action of $\Delta_{X}$ on $\Delta_{X / S}(n / n+1)$ (induced via conjugation) is trivial for any $n \geq 1$.

$\left(1^{\prime}\right)$ The action of $\Delta_{S}$ on $\Delta_{X / S}(n / n+1)$ (induced via $\left.\rho_{X / S}\right)$ is trivial for any $n \geq 1$

(2) The action of $\Delta_{X}$ on $\left(\Delta_{X / S}^{\mathrm{cpt}}\right)^{\mathrm{ab}}$ and $I^{\mathrm{cpt}} /\left(\Delta_{X / S}(3) \cap I^{\mathrm{cpt}}\right)$ (induced via conjugation) is trivial.

$\left(2^{\prime}\right)$ The action of $\Delta_{S}$ on $\left(\Delta_{X / S}^{\mathrm{cpt}}\right)^{\mathrm{ab}}$ and $I^{\mathrm{cpt}} /\left(\Delta_{X / S}(3) \cap I^{\mathrm{cpt}}\right)$ (induced via $\left.\rho_{X / S}\right)$ is trivial.

(ii) The submodule

$$
I^{\mathrm{cpt}} /\left(\Delta_{X / S}(3) \cap I^{\mathrm{cpt}}\right) \subseteq \Delta_{X / S}(2 / 3)
$$

is a free $\mathbb{Z}_{l}$-module in the formal generators $\zeta$, where $\zeta$ ranges over the elements of the underlying set of $D_{\bar{s}}$. Moreover, the action of $\Delta_{S}$ on the generators $\zeta$ of $I^{\mathrm{cpt}} /\left(\Delta_{X / S}(3) \cap I^{\mathrm{cpt}}\right)$ (induced via $\left.\rho_{X / S}\right)$ is compatible with the natural action of $\Delta_{S}$ on $D_{\bar{s}}$.

Proof. This follows immediately from [8], Proposition 1.

Corollary 1.1. Let $A_{X}$ be a profinite abelian group, and $\Delta_{X} \rightarrow A_{X}$ a surjection. If $\left(f, \Delta_{X} \rightarrow A_{X}, \Delta_{S} \rightarrow \Delta_{S}^{\mathrm{ab}}\right)$ satisfies condition (ii) in Definition 1.3 , and the action of $\pi_{1}(S)$ on $\left(\Delta_{X / S}^{\mathrm{cpt}}\right)^{\mathrm{ab}}$ and on $I^{\mathrm{cpt}} / \Delta_{X / S}(3) \cap I^{\mathrm{cpt}}$ (induced via $\left.\rho_{X / S}\right)$ are trivial, then $\left(f, \Delta_{X} \rightarrow A_{X}, \Delta_{S} \rightarrow \Delta_{S}^{\mathrm{ab}}\right)$ is of Lie-exact type.

Proof. This follows immediately from Propositions 1.2; 1.3; Lemma 1.2, together with the well-known identity due to P. Hall applied in the proof of Proposition 1.3.

Definition 1.4. Let $m$ be a natural number.

(i) We shall say that a sequence of morphisms of schemes

$$
X_{m} \stackrel{f_{m-1}}{\longrightarrow} X_{m-1} \stackrel{f_{m-2}}{\longrightarrow} \cdots \stackrel{f_{1}}{\longrightarrow} X_{1} \stackrel{f_{0}}{\longrightarrow} X_{0}=\operatorname{Spec} K
$$

over the separably closed field $K$ is a successive extension of hyperbolic curves of product type if there exist proper hyperbolic curves $C_{i}$ (where $i=$ $0, \cdots, m-1)$ over $K$ which satisfy the following condition: The morphism $f_{i}: X_{i+1} \rightarrow X_{i}$ factors as the composite

$$
X_{i+1} \hookrightarrow C_{i} \times_{K} X_{i} \stackrel{\mathrm{pr}_{2}}{\longrightarrow} X_{i},
$$


where the first arrow is an open immersion $X_{i+1} \hookrightarrow C_{i} \times_{K} X_{i}$ onto the complement $\left(C_{i} \times_{K} X_{i}\right) \backslash D_{i}$ of a relative divisor $D_{i}$ which is finite étale over $X_{i}$.

Note that it is immediate that $X_{i}$ is a regular scheme of dimension $i$, that $f_{i}$ is a family of hyperbolic curves, and that the $f_{i}$ 's induce an open immersion $X_{i} \hookrightarrow C_{0} \times_{K} \cdots \times_{K} C_{i-1}$.

(ii) Let

$$
X_{m} \stackrel{f_{m-1}}{\longrightarrow} X_{m-1} \stackrel{f_{m-2}}{\longrightarrow} \cdots \stackrel{f_{1}}{\longrightarrow} X_{1} \stackrel{f_{0}}{\longrightarrow} X_{0}=\operatorname{Spec} K
$$

be a successive extension of hyperbolic curves of product type. Then we shall denote by

$$
\left\{\Delta_{X_{i}}(n)\right\}
$$

the central filtration of the pro-l fundamental group $\Delta_{X_{i}}$ of $X_{i}$ with respect to the composite of the natural surjections

$$
\Delta_{X_{i}} \rightarrow \Delta_{C_{0} \times_{K} \cdots \times_{K} C_{i-1}} \rightarrow \Delta_{C_{0} \times_{K} \cdots \times_{K} C_{i-1}}^{\mathrm{ab}}\left(\simeq \Delta_{C_{0}}^{\mathrm{ab}} \times \cdots \times \Delta_{C_{i-1}}^{\mathrm{ab}}\right),
$$

where the first arrow is the morphism induced by the open immersion $X_{i} \hookrightarrow C_{0} \times_{K} \cdots \times_{K} C_{i-1}$ (cf. (i)).

Note that it is immediate that the following sequence is exact:

$$
1 \longrightarrow \Delta_{X_{i+1} / X_{i}}(1 / 2) \longrightarrow \Delta_{X_{i+1}}(1 / 2) \stackrel{\text { via } f_{i}}{\longrightarrow} \Delta_{X_{i}}(1 / 2) \longrightarrow 1 .
$$

\section{Proposition 1.4. Let}

$$
X_{m} \stackrel{f_{m-1}}{\longrightarrow} X_{m-1} \stackrel{f_{m-2}}{\longrightarrow} \cdots \stackrel{f_{1}}{\longrightarrow} X_{1} \stackrel{f_{0}}{\longrightarrow} X_{0}=\operatorname{Spec} K
$$

be a successive extension of hyperbolic curves of product type, and $0 \leq i \leq m-1$ an integer. Then the following hold:

(i) The morphism $f_{i}$ is of pro-l-exact type.

(ii) The following conditions are equivalent:

(1) The relative divisor $D_{i}$ (which appears in Definition 1.4, (i)) is empty or the finite étale covering $D_{i} \rightarrow X_{i}$ is trivial (i.e., $D_{i}$ is a disjoint union of copies of $X_{i}$, and the covering $D_{i} \rightarrow X_{i}$ is induced by the identity morphism of $X_{i}$ ).

(2) $\left(f_{i}, \Delta_{X_{i+1}} \rightarrow \Delta_{X_{i+1}}(1 / 2), \Delta_{X_{i}} \rightarrow \Delta_{X_{i}}(1 / 2)\right)$ is of Lie-exact type. 
Proof. First, we prove assertion (i). Since the diagram

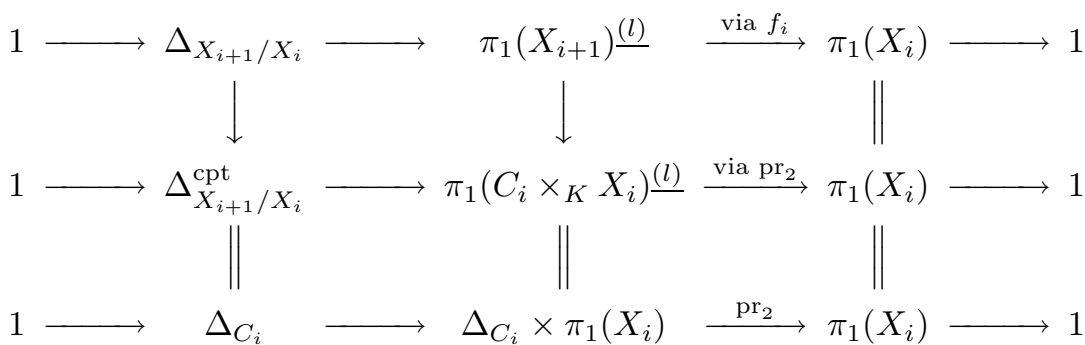

commutes, the action of $\pi_{1}\left(X_{i}\right)$ on $\Delta_{X_{i+1} / X_{i}}^{\mathrm{cpt}}$ is trivial; thus, assertion (i) follows from Proposition 1.2.

Next, we prove assertion (ii). Assume that condition (1) holds. Then, by Lemma 1.2, (ii), the action of $\Delta_{X_{i}}$ on $I^{\mathrm{cpt}} /\left(\Delta_{X_{i+1} / X_{i}}(3) \cap I^{\mathrm{cpt}}\right)$ is trivial. Thus, in light of the triviality of the action of $\pi_{1}\left(X_{i}\right)$ on $\Delta_{X_{i+1} / X_{i}}^{\mathrm{cpt}}$ (observed in the proof of assertion (i)), we conclude that the action of $\Delta_{X_{i}}$ on $\Delta_{X_{i+1} / X_{i}}(n / n+$ 1 ) is trivial for any $n \geq 1$ (cf. Lemma 1.2, (i)). Thus, it follows from the equivalence of (i) and (ii') in Proposition 1.3 that it is enough to show that the action of $\Delta_{X_{i}}(2)$ on $\Delta_{X_{i+1} / X_{i}}(n / n+2)$ is trivial for any $n \geq 1$. Moreover, by the triviality of the action of $\pi_{1}\left(X_{i}\right)$ on $\Delta_{X_{i+1} / X_{i}}^{\mathrm{cpt}}$ (observed in the proof of (i)), together with the well-known identity due to P. Hall applied in the proof of Proposition 1.3, the action of $\left[\Delta_{X_{i}}, \Delta_{X_{i}}\right]$ on $\Delta_{X_{i+1} / X_{i}}(n / n+2)$ is trivial for any $n \geq 1$. Since $\Delta_{X_{i}}(2)$ is generated by $\left[\Delta_{X_{i}}, \Delta_{X_{i}}\right]$ and the kernel $I$ of the natural surjection $\Delta_{X_{i}} \rightarrow \Delta_{C_{0} \times_{K} \cdots \times_{K} C_{i-1}}\left(\simeq \Delta_{C_{0}} \times \cdots \times \Delta_{C_{i-1}}\right)$, it is enough to show that the action of $I$ on $\Delta_{X_{i+1} / X_{i}}(n / n+2)$ is trivial for any $n \geq 1$. Therefore, if the natural inclusion $X_{i} \hookrightarrow C_{0} \times_{K} \cdots \times_{K} C_{i-1}$ is an isomorphism, then the assertion follows.

Assume that $X_{i} \hookrightarrow C_{0} \times_{K} \cdots \times_{K} C_{i-1}$ is not an isomorphism. Then since $I$ is topologically normally generated by the inertia subgroups (well-defined, up to conjugation) of $\Delta_{X_{i}}$ determined by the irreducible components of the divisor with normal crossings $\left(C_{0} \times_{K} \cdots \times_{K} C_{i-1}\right) \backslash X_{i} \subseteq C_{0} \times_{K} \cdots \times_{K} C_{i-1}$ (by the purity theorem [cf. [4], Exposé X, Theorem 3.4], together with the regularity of $\left.C_{0} \times{ }_{K} \cdots \times_{K} C_{i-1}\right)$, it is enough to show the following assertion:

$(\dagger)$ : The action of these inertia subgroups on $\Delta_{X_{i+1} / X_{i}}(n / n+2)$ is trivial for any $n \geq 1$.

For any positive integer $N$, we shall denote by $C_{i(N)}$ (respectively, $U_{C_{i(N)}}$ ) the fiber product of $N$ copies of $C_{i}$ over Spec $K$ (respectively, the $N$-th configuration space of $C_{i}$, i.e., the scheme which represents the open subfunctor

$$
S \mapsto\left\{\left(s_{1}, \cdots, s_{N}\right) \in C_{i \underline{(N)}}(S)=C_{i}(S)^{\times N} \mid s_{n} \neq s_{m} \text { if } n \neq m\right\}
$$


of the functor represented by $\left.C_{i(N)}\right)$. By (1), if we denote by $r$ the degree of the (trivial) covering $D_{i} \rightarrow \overline{X_{i}}$, then there exist "classifying morphisms" $X_{i} \stackrel{g_{i}}{\rightarrow} U_{C_{i(r)}}$ and $X_{i+1} \stackrel{g_{i+1}}{\rightarrow} U_{C_{i(r+1)}}$ that fit into the following cartesian diagram

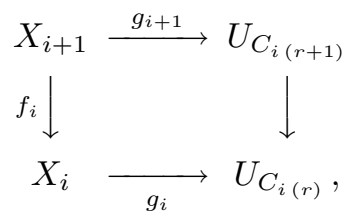

where the right-hand vertical arrow is the morphism induced by the morphism $C_{i(r+1)} \rightarrow C_{i(r)}$ obtained by forgetting the $(r+1)$-st factor. (In fact, one can regard $U_{C_{i(r)}}$ as a moduli scheme of curves obtained as the complement in $C_{i}$ of distinct $r$ points; moreover, one can regard the right-hand vertical arrow in the above diagram as a universal object.) Thus, we obtain a commutative diagram

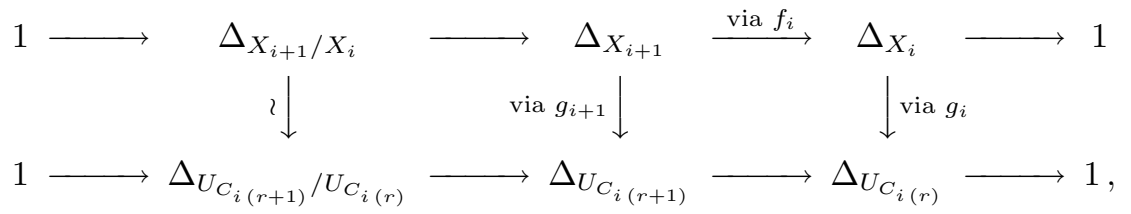

where the horizontal sequences are exact, and the left-hand vertical arrow is an isomorphism. Note that the sequence

$$
U_{C_{i(r)}} \longrightarrow U_{C_{i(r-1)}} \longrightarrow \cdots \longrightarrow U_{C_{i(2)}} \longrightarrow C_{i} \longrightarrow \operatorname{Spec} K
$$

(where the morphism $U_{C_{i(N+1)}} \longrightarrow U_{C_{i(N)}}$ [where $\left.1 \leq N \leq r-1\right]$ is the morphism induced by the morphism $C_{i(N+1)} \rightarrow C_{i(N)}$ obtained by forgetting the $(N+1)$-st factor) is a successive extension of hyperbolic curves of product type; thus, the filtration $\left\{\Delta_{U_{C_{i(r)}}}(n)\right\}$ is defined (cf. Definition 1.4, (ii)). Moreover, since the sequence

$$
1 \longrightarrow \operatorname{Gr}\left(\Delta_{U_{C_{i(r+1)}} / U_{C_{i(r)}}}\right) \longrightarrow \operatorname{Gr}\left(\Delta_{U_{C_{i(r+1)}}}\right) \longrightarrow \operatorname{Gr}\left(\Delta_{U_{C_{i(r)}}}\right) \longrightarrow 1
$$

(naturally induced by the bottom sequence in the commutative diagram $(*)$ ) is exact (cf. [12], Proposition 3.2, (i)), by the equivalence in Proposition 1.3, (i) and (ii'), the action of $\Delta_{U_{C_{i(r)}}}(2)$ on $\Delta_{U_{C_{i(r+1)}} / U_{C_{i(r)}}}(n / n+2)$ is trivial for any $n \geq 1$. Thus, by the commutativity of the above diagram $(*)$ and the fact that the left-hand vertical arrow in the above diagram $(*)$ is an isomorphism, to prove the assertion that condition (1) implies condition (2), it is enough to show that the composite $X_{i} \stackrel{g_{i}}{\rightarrow} U_{C_{i(r)}} \hookrightarrow C_{i(r)}$ extends to the generic points of the irreducible components of the divisor with normal crossings $\left(C_{0} \times_{K} \cdots \times_{K}\right.$ 
$\left.C_{i-1}\right) \backslash X_{i} \subseteq C_{0} \times_{K} \cdots \times_{K} C_{i-1}$. On the other hand, this follows from the properness of $C_{i(r)}$. This completes the proof of the assertion that condition (1) implies condition (2).

Next, assume that $\left(f_{i}, \Delta_{X_{i+1}} \rightarrow \Delta_{X_{i+1}}(1 / 2), \Delta_{X_{i}} \rightarrow \Delta_{X_{i}}(1 / 2)\right)$ is of Lieexact type. Then the equivalence of (i) and (ii') in Proposition 1.3 and the equivalence of $\left(1^{\prime}\right)$ and $\left(2^{\prime}\right)$ in Lemma 1.2, (i), imply that the action of $\Delta_{X_{i}}$ on $I^{\mathrm{cpt}} /\left(\Delta_{X_{i+1} / X_{i}}(3) \cap I^{\mathrm{cpt}}\right)$, where $I^{\mathrm{cpt}}$ is the kernel of the natural surjection $\Delta_{X_{i+1} / X_{i}} \rightarrow \Delta_{X_{i+1} / X_{i}}^{\mathrm{cpt}}$, is trivial. Therefore, by Lemma 1.2, (ii), we conclude that either the relative divisor $D_{i}$ is empty, or the finite étale covering $D_{i} \rightarrow X_{i}$ is trivial.

Remark 3. Note that the exactness of the sequence $(* *)$ in the proof of Proposition 1.4 can also be proven as follows. Note that we showed the exactness of the sequence $(* *)$ in the proof of Proposition 1.4 by means of [12], Proposition 3.2, (i), which is proven via transcendental techniques; however, the following proof is purely algebraic:

To prove the exactness of the sequence $(* *)$, by a similar argument to the argument used in the proof of Proposition 1.4, it is enough to show the assertion $(\dagger)$ in the proof of Proposition 1.4, i.e., the action of the inertia subgroups of $\Delta_{U_{C_{i(r)}}}$ on $\Delta_{U_{C_{i(r+1)}} / U_{C_{i(r)}}}(n / n+2)$ is trivial for any $n \geq 1$. Moreover, since this problem manifestly depends only on purely group-theoretic data of the sequence

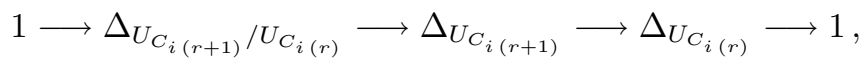

we may assume without loss of generality that there exists a finite field $k$ such that the proper hyperbolic curve " $C_{i}$ " are defined over $k$. We shall write $X_{i+1} \stackrel{\text { def }}{=} U_{C_{i(r+1)}}$ and $X_{i} \stackrel{\text { def }}{=} U_{C_{i(r)}}$; moreover, we shall denote by $G_{k}$ the absolute Galois group of $k$, by $\operatorname{Fr}_{k} \in G_{k}$ the Frobenius element, and by $q_{k}$ the cardinality of $k$. Then by the "Riemann hypothesis for abelian varieties over finite fields" (cf. e.g., [18], p. 206) (respectively, as is well-known), the eigenvalues of the action of $\mathrm{Fr}_{k}$ on the $G_{k}$-module $\Delta_{X_{i+1} / X_{i}}(n / n+1)$ (respectively, the inertia subgroup) are algebraic numbers all of whose complex absolute values are equal to $q_{k}^{n / 2}$ (respectively, $q_{k}$ ), i.e., the $G_{k}$-module $\Delta_{X_{i+1} / X_{i}}(n / n+1)$ (respectively, the inertia subgroup) is "of weight $n$ " (respectively, "of weight $2 ")$. In particular, the $G_{k}$-module

$$
\operatorname{Hom}_{G_{k}}\left(\Delta_{X_{i+1} / X_{i}}(n / n+1), \Delta_{X_{i+1} / X_{i}}(n+1 / n+2)\right)
$$

is "of weight 1". On the other hand, since the action of the inertia subgroup on $\Delta_{X_{i+1} / X_{i}}(n / n+1)$ and $\Delta_{X_{i+1} / X_{i}}(n+1 / n+2)$ is trivial, by the exactness 
of the sequence

$1 \longrightarrow \Delta_{X_{i+1} / X_{i}}(n+1 / n+2) \longrightarrow \Delta_{X_{i+1} / X_{i}}(n / n+2) \longrightarrow \Delta_{X_{i+1} / X_{i}}(n / n+1) \longrightarrow 1$,

the action of the inertia subgroup on $\Delta_{X_{i+1} / X_{i}}(n / n+2)$ determines (and is determined by!) a $G_{k}$-equivariant homomorphism from the inertia subgroup to

$$
\operatorname{Hom}_{G_{k}}\left(\Delta_{X_{i+1} / X_{i}}(n / n+1), \Delta_{X_{i+1} / X_{i}}(n+1 / n+2)\right) .
$$

Thus, by considering the "weights" of the domain and codomain of this $G_{k^{-}}$ equivariant homomorphism, we conclude that the $G_{k}$-equivariant homomorphism is trivial; in particular, the action of the inertia subgroup on $\Delta_{X_{i+1} / X_{i}}(n / n+2)$ is trivial.

\section{$\S 2$. Fundamental Groups of Configuration Spaces over Finite Fields}

In this Section, we consider the group-theoretic properties of the fundamental groups of configuration spaces.

Let $K$ be a field, and $l$ a prime number that is invertible in $K$. We shall fix a separable closure $K^{\text {sep }}$ of $K$. We shall denote by $G_{K}$ the Galois group of $K^{\text {sep }}$ over $K$. Moreover, in the following, let $X$ be a proper hyperbolic curve of genus $g_{X} \geq 2$ over $K$.

Definition 2.1. Let $r$ be a natural number.

(i) We shall denote by $X_{(r)}$ the fiber product of $r$ copies of $X$ over $\operatorname{Spec} K$, i.e.,

$$
X_{\underline{(r)}} \stackrel{\text { def }}{=} \overbrace{X \times_{K} \cdots \times_{K} X}^{r} .
$$

For an integer $1 \leq i \leq r$, we shall denote by $p_{X_{(r-1)}: i}: X_{\underline{(r)}} \rightarrow X_{\underline{(r-1)}}$ the morphism obtained by forgetting the $i$-th factor.

(ii) We shall denote by $U_{X_{(r)}} \subseteq X_{(r)}$ the $r$-th configuration space of $X$, i.e., the scheme which represents the open subfunctor

$$
S \mapsto\left\{\left(f_{1}, \cdots, f_{r}\right) \in X_{\underline{(r)}}(S)=X(S)^{\times r} \mid f_{i} \neq f_{j} \text { if } i \neq j\right\}
$$

of the functor represented by $X_{(r)}$. For an integer $1 \leq i \leq r$, we shall denote by $p_{U_{X_{(r-1)}}: i}: U_{X_{(r)}} \rightarrow U_{X_{(r-1)}}$ the morphism induced by $p_{X_{(r-1)}: i}$. 
Let $1 \leq i<j \leq r$ be integers. Then we shall denote by $D_{X_{(r)}\{i, j\}} \subseteq X_{(r)}$ the closed subscheme of $X_{(r)}$ which represents the closed subfunctor

$$
S \mapsto\left\{\left(f_{1}, \cdots, f_{r}\right) \in X_{\underline{(r)}}(S)=X(S)^{\times r} \mid f_{i}=f_{j}\right\}
$$

of the functor represented by $X_{\underline{(r)}}$. Then it is immediate that

$$
U_{X_{(r)}}=X_{\underline{(r)}} \backslash \bigcup_{1 \leq i<j \leq r} D_{X_{\underline{(r)}}\{i, j\}} .
$$

(iii) We shall denote by $\Pi_{X_{(r)}}$ the geometrically pro-l fundamental group of $X_{\underline{(r)}}$, and by $\Delta_{X_{\underline{(r)}}}$ the kernel of the natural surjection

$$
\Pi_{X_{\underline{(r)}}} \rightarrow G_{K} .
$$

Thus, we have an exact sequence

$$
1 \longrightarrow \Delta_{X_{\underline{(r)}}} \longrightarrow \Pi_{X_{\underline{(r)}}} \longrightarrow G_{K} \longrightarrow 1 .
$$

Moreover, we shall write

$$
\Pi_{X} \stackrel{\text { def }}{=} \Pi_{X_{(1)}} ; \quad \Delta_{X} \stackrel{\text { def }}{=} \Delta_{X_{(1)}} .
$$

(iv) We shall denote by $\Pi_{X_{(r)}}$ the geometrically pro-l fundamental group of $U_{X_{(r)}}$, by $\Delta_{X_{(r)}}$ the kernel of the natural surjection

$$
\Pi_{X_{(r)}} \rightarrow G_{K}
$$

and by $\Delta_{X_{(r)} / X_{(r-1)}}^{(i)}$ the kernel of the surjection

$$
\Delta_{X_{(r)}} \stackrel{\text { via }}{p_{U_{X}}} \rightarrow{ }^{: i} \Delta_{X_{(r-1)}}(i=1, \cdots, r) .
$$

Thus, we have exact sequences

$$
\begin{gathered}
1 \longrightarrow \Delta_{X_{(r)}} \longrightarrow \Pi_{X_{(r)}} \longrightarrow G_{K} \longrightarrow 1 ; \\
1 \longrightarrow \Delta_{X_{(r)} / X_{(r-1)}}^{(i)} \longrightarrow \Delta_{X_{(r)}} \stackrel{\text { via }}{p_{\left.U_{X_{(r-1)}}\right)^{i i}}} \Delta_{X_{(r-1)}} \longrightarrow 1 ; \\
1 \longrightarrow \Delta_{X_{(r)} / X_{(r-1)}}^{(i)} \longrightarrow \Pi_{X_{(r)}} \stackrel{\text { via }}{\longrightarrow p_{U_{X_{(r-1)}}: i}} \Pi_{X_{(r-1)}} \longrightarrow 1 .
\end{gathered}
$$

Note that since the sequence obtained as the base-change of

$$
U_{X_{(r)}} \stackrel{p_{U_{X_{(r-1)}}}: r}{\longrightarrow} U_{X_{(r-1)}} \stackrel{p_{U_{X_{(r-2)}}: r-1}^{\longrightarrow}}{\longrightarrow} \stackrel{p_{U_{X_{(1)}}: 2}}{\longrightarrow} X \longrightarrow \operatorname{Spec} K
$$


from $K$ to $K^{\text {sep }}$ is a successive extension of hyperbolic curves of product type (cf. Definition 1.4, (i)), the family of curves $U_{X_{(r)}} \otimes_{K} K^{\text {sep }} \stackrel{\text { via }}{p_{U_{X}}} \rightarrow$ $U_{X_{(r-1)}} \otimes_{K} K^{\text {sep }}$ is of pro-l exact type (cf. Proposition 1.4, (i)); thus, the pro-l group $\Delta_{X_{(r)} / X_{(r-1)}}^{(i)}$ is isomorphic to the pro-l fundamental group of the geometric fiber of the family of curves $U_{X_{(r)}} \otimes_{K} K^{\text {sep }} \stackrel{\text { via }}{p_{U_{X}(r-1)}} \rightarrow$ $U_{X_{(r-1)}} \otimes_{K} K^{\text {sep }}$ at a geometric point of $U_{X_{(r-1)}} \otimes_{K} K^{\text {sep }}$.

Proposition 2.1. $\quad$ Let $r$ be a positive integer. Then the profinite groups $\Delta_{X_{(r)}}, \Delta_{X_{(r)}}$, and $\Delta_{X_{(r)} / X_{(r-1)}}^{(i)}$ are slim (where we refer to the discussion entitled "Groups" in Introduction concerning the term "slim").

Proof. The slimness of $\Delta_{X_{(r)} / X_{(r-1)}}^{(i)}$ (in particular, the slimness of $\Delta_{X}$ ) follows from [1], Propositions 8; 18. The slimness of $\Delta_{X_{\underline{(r)}}}$ follows from the slimness of $\Delta_{X}$, together with the fact that $\Delta_{X_{\underline{(r)}}}$ is the product of $r$ copies of $\Delta_{X}$. The slimness of $\Delta_{X_{(r)}}$ follows from induction on $r$, the slimness of $\Delta_{X_{(r)} / X_{(r-1)}}^{(i)}$, and the exactness of the sequence

$$
1 \longrightarrow \Delta_{X_{(r)} / X_{(r-1)}}^{(i)} \longrightarrow \Delta_{X_{(r)}} \stackrel{\text { via } p_{U_{X_{(r-1)}}: i}}{\longrightarrow} \Delta_{X_{(r-1)}} \longrightarrow 1
$$

in Definition 2.1, (iv) (cf. the discussion entitled "Groups" in Introduction).

Next, let us recall the theory of log configuration schemes (cf. [7], Section 2). Let us denote by $X_{(r)}^{\log }$ the $r$-th $\log$ configuration scheme of $X$, i.e.,

$$
X_{(r)}^{\log } \stackrel{\text { def }}{=} \operatorname{Spec} K \times \overline{\mathcal{M}}_{g}^{\log } \overline{\mathcal{M}}_{g, r}^{\log }
$$

(where we refer to the discussion entitled "Curves" in Introduction concerning $\overline{\mathcal{M}}_{g, r}^{\log }$ ), where the (1-)morphism Spec $K \rightarrow \overline{\mathcal{M}}_{g}^{\log }$ is the classifying morphism of the curve $X \rightarrow \operatorname{Spec} K$, and the (1-)morphism $\overline{\mathcal{M}}_{g, r}^{\log } \rightarrow \overline{\mathcal{M}}_{g}^{\log }$ is the (1-)morphism obtained by forgetting the sections; and by $p_{X_{(r-1)}: i}^{\log }: X_{(r)}^{\log } \rightarrow$ $X_{(r-1)}^{\log }$ the morphism induced by the (1-)morphism $\overline{\mathcal{M}}_{g, r}^{\log } \rightarrow \overline{\mathcal{M}}_{g, r-1}^{\log }$ obtained by forgetting the $i$-th section (cf. [7], Definitions 1 and 2). Then the following holds:

Proposition 2.2.

(i) The interior (where we refer to the discussion entitled "Log schemes" in Introduction concerning the term "interior") of the log scheme $X_{(r)}^{\log }$ is naturally isomorphic to the usual $r$-th configuration space $U_{X_{(r)}}$ of $X$. 
(ii) The log scheme $X_{(r)}^{\log }$ is connected, regular, and log regular.

(iii) The morphism $p_{X_{(r-1)} \text { : }}^{\log }$ is proper, flat, geometrically connected, geometrically reduced, and log smooth.

Proof. Assertion (i) (respectively, (ii); respectively, (iii)) follows from the definition of $X_{(r)}^{\log }$ (respectively, [7], Proposition 2.1, (i), (iii), and (iv); respectively, the definition of $p_{X_{(r-1)}: i}^{\log }$, together with [7], Proposition 2.1, (ii)).

Now we have a natural commutative diagram

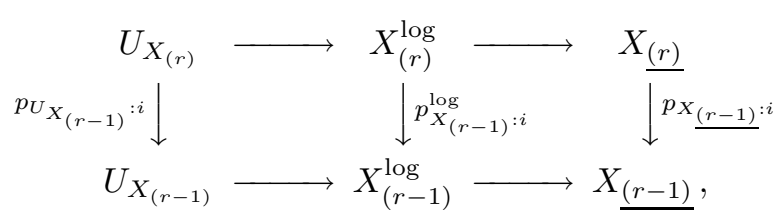

where the right-hand horizontal arrows are the morphisms obtained as the composites of the morphisms " $X_{(-)}^{\log } \rightarrow X_{(-)}$" obtained by forgetting the log structures and the natural morphisms " $X_{(-)} \rightarrow X_{(-)}$". This diagram induces a sequence

$$
\Pi_{X_{(r)}} \longrightarrow \pi_{1}\left(X_{(r)}^{\log }\right) \underline{(l)} \longrightarrow \Pi_{X_{(r)}},
$$

where $\pi_{1}\left(X_{(r)}^{\log }\right) \underline{(l)}$ is the geometrically pro- $l \log$ fundamental group of $X_{(r)}^{\log }$. Now by [7], Lemma 4.2, (i) (i.e., Proposition 2.2, (i), (ii), together with the log purity theorem), the first morphism $\Pi_{X_{(r)}} \rightarrow \pi_{1}\left(X_{(r)}^{\log }\right) \underline{(l)}$ (in the above sequence) is an isomorphism. In the following, we identify $\Pi_{X_{(r)}}$ with $\pi_{1}\left(X_{(r)}^{\log }\right) \frac{(l)}{b}$ by means of this isomorphism.

Let $I$ be a subset of $\{1,2, \cdots, r\}$ of cardinality $I^{\#} \geq 2$. We denote by $D_{X_{(r)} I}^{\log }$ the log scheme defined in [7], Definition 3, i.e., the log scheme obtained as follows: By applying the clutching (1-)morphism (cf. [10], Definition 3.8)

$$
\beta_{g, 0,\{1,2, \cdots, r\} \backslash I, I}: \overline{\mathcal{M}}_{g, r-I^{\#}+1} \times_{K} \overline{\mathcal{M}}_{0, I^{\#}+1} \rightarrow \overline{\mathcal{M}}_{g, r}
$$

(where $I$ and $\{1,2, \cdots, r\} \backslash I$ are equipped with the natural ordering) to the $\left(r-I^{\#}+1\right)$-pointed stable curve of genus $g$

$$
X_{\left(r-I^{\#}+2\right)} \times_{K} \overline{\mathcal{M}}_{0, I^{\#}+1} \longrightarrow X_{\left(r-I^{\#}+1\right)} \times_{K} \overline{\mathcal{M}}_{0, I^{\#}+1}
$$

obtained by base-changing $X_{\left(r-I^{\#}+2\right)} \stackrel{p_{X}}{\stackrel{(r-I \#+1)}{\rightarrow}}{ }^{r-I^{\#}+2} X_{\left(r-I^{\#}+1\right)}$ and the $\left(I^{\#}+\right.$ 1)-pointed stable curve of genus 0

$$
X_{\left(r-I^{\#}+1\right)} \times_{K} \overline{\mathcal{M}}_{0, I^{\#}+2} \longrightarrow X_{\left(r-I^{\#}+1\right)} \times_{K} \overline{\mathcal{M}}_{0, I^{\#}+1}
$$


obtained by base-changing the universal curve $\overline{\mathcal{M}}_{0, I^{\#+2}} \rightarrow \overline{\mathcal{M}}_{0, I \#+1}$ over $\overline{\mathcal{M}}_{0, I \#+1}$, we obtain an $r$-pointed stable curve of genus $g$. Then it is immediate that the classifying (1-)morphism $X_{\left(r-I^{\#}+1\right)} \times_{K} \overline{\mathcal{M}}_{0, I^{\#}+1} \rightarrow \overline{\mathcal{M}}_{g, r}$ of this $r$-pointed stable curve of genus $g$ factors through $X_{(r)}$, and this morphism $X_{\left(r-I^{\#+1)}\right.} \times_{K} \overline{\mathcal{M}}_{0, I^{\#+1}} \rightarrow X_{(r)}$ is a closed immersion. We shall denote by $\delta_{X_{(r)} I}$ this closed immersion, by $D_{X_{(r)} I}$ the scheme-theoretic image of $\delta_{X_{(r)} I}$, by $D_{X_{(r)} I}^{\log }$ the $\log$ scheme obtained by equipping $D_{X_{(r)} I}$ with the log structure induced by the $\log$ structure of $X_{(r)}^{\log }$, and by $\delta_{X_{(r)} I}^{\log }: D_{X_{(r)} I}^{\log } \rightarrow X_{(r)}^{\log }$ the strict closed immersion whose underlying morphism is $\delta_{X_{(r)} I}$. Then the following holds:

\section{Proposition 2.3.}

(i) The underlying scheme of the log scheme $D_{X_{(r)} I}^{\log }$ is isomorphic to $X_{(r-I \#+1)}$ $\times_{K} \overline{\mathcal{M}}_{0, I}{ }^{\#+1}$.

(ii) The log structure of the log scheme $X_{(r)}^{\log }$ is the log structure defined by the divisor with normal crossings $\sum_{I \# \geq 2} D_{X_{(r)} I} \subseteq X_{(r)}$.

Proof. Assertion (i) (respectively, (ii)) follows from the definition of $D_{X_{(r)} I}^{\log }$ (respectively, [7], Proposition 3.1, (ii)).

Now if $1 \leq i<j \leq r$ are integers, then it follows from [7], Proposition 3.2, (i), together with definitions, that $p_{X_{(r)}: i}^{\log } \circ \delta_{X_{(r+1)}\{i, j\}}^{\log }=p_{X_{(r)}: j}^{\log } \circ \delta_{X_{(r+1)}\{i, j\}}^{\log }$ is a morphism of type $\mathbb{N}$, i.e., the underlying morphism of schemes is an isomorphism, and the relative characteristic sheaf (where we refer to the discussion entitled "Log schemes" in Introduction concerning the term "relative characteristic sheaf") is locally constant with stalk isomorphic to $\mathbb{N}$ (cf. [6], Definition 6). Let $\bar{x} \rightarrow X_{(r)}^{\log }$ be a geometric point whose image lies in the interior $U_{X_{(r)}}$ of $X_{(r)}^{\log }$. Then we obtain the following commutative diagram:

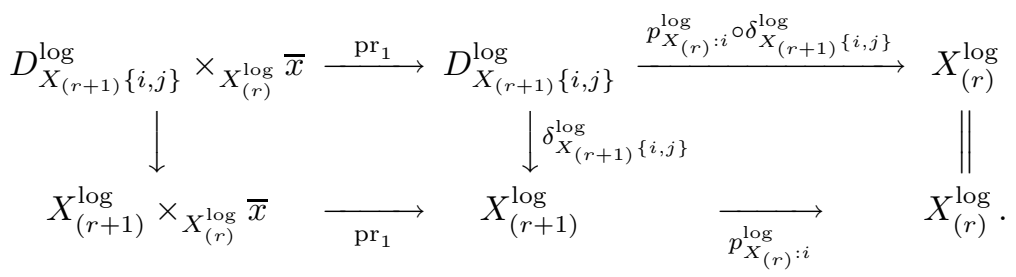


This diagram induces a commutative diagram

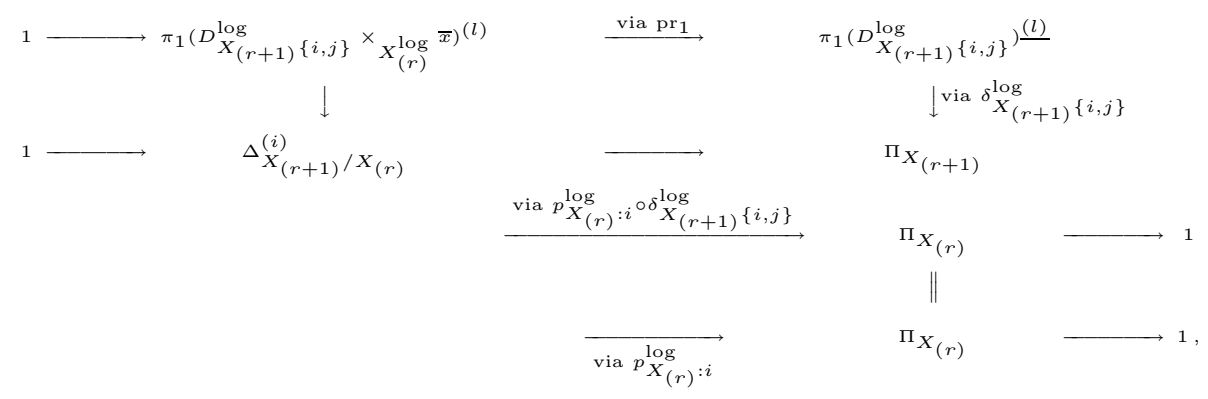

where the horizontal sequences are exact (cf. [6], Proposition 4; Remark 15; [7], Lemma 4.2, (iii)), and we have $\pi_{1}\left(D_{X_{(r+1)}\{i, j\}}^{\log } \times_{X_{(r)}^{\log } \bar{x}}^{(l)} \stackrel{\sim}{\rightarrow} \mathbb{Z}_{l}(1)\right.$ (where "(1)" denotes a Tate twist); moreover, by the definition of $D_{X_{(r+1)}\{i, j\}}^{\log }$, it follows that the left-hand vertical arrow $\pi_{1}\left(D_{X_{(r+1)}\{i, j\}}^{\log } \times_{X_{(r)}^{\log }} \overline{)^{(l)}} \rightarrow \Delta_{X_{(r+1)} / X_{(r)}}^{(i)}\right.$ is injective, and this image is the inertia subgroup (well-defined, up to conjugation)

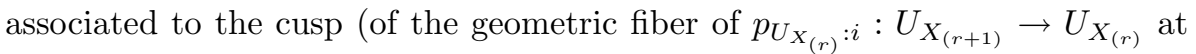
a geometric point of $U_{X_{(r)}}$ ) determined by the divisor $D_{X_{(r+1)}\{i, j\}} \subseteq X_{\underline{(r+1)}}$. In particular, the vertical arrow $\pi_{1}\left(D_{X_{(r+1)}\{i, j\}}^{\log }\right) \stackrel{(l)}{\rightarrow} \Pi_{X_{(r+1)}}$ in the above diagram is also injective.

Definition 2.2. Let $r \geq 2$ be an integer, and $I$ a subset of $\{1,2, \cdots, r\}$ of cardinality $I^{\#} \geq 2$. Then we shall denote by $\mathfrak{D}_{X_{(r)} I}$ the image of the morphism $\pi_{1}\left(D_{X_{(r)} I}^{\log }\right) \stackrel{(l)}{\rightarrow} \Pi_{X_{(r)}}$ induced by $\delta_{X_{(r)} I}^{\log }$, where $\pi_{1}\left(D_{X_{(r)} I}^{\log }\right) \underline{(l)}$ is the geometrically pro-l $l \log$ fundamental group of $D_{X_{(r)} I}^{\log }$. We shall denote by $\mathfrak{D}_{X_{(r)} I}^{\Delta}$ the intersection of $\mathfrak{D}_{X_{(r)} I}$ and $\Delta_{X_{(r)}}$. Note that these subgroups are welldefined, up to conjugation.

Moreover, if $I^{\#} \geq 3$, then by [7], Proposition 3.1, (v), the composite

$$
D_{X_{(r)} I}^{\log } \stackrel{\delta_{X}^{\log }}{\stackrel{(r) I}{\longrightarrow}} X_{(r)}^{\log } \stackrel{p_{X}^{\log }}{p_{(r-1)}^{: i}} X_{(r-1)}^{\log }
$$

factors through $\delta_{X_{(r-1)} I^{[i]}}^{\log }: D_{X_{(r-1)} I^{[i]}}^{\log } \hookrightarrow X_{(r-1)}^{\log }$, where $I^{[i]}$ is the unique subset of $\{1,2, \cdots, r-1\}$ such that for $1 \leq j \leq r-1, j \in I^{[i]}$ if and only if

$$
\left\{\begin{array}{r}
j \in I \text { if } j<i \\
j+1 \in I \text { if } j \geq i
\end{array}\right.
$$

On the other hand, by [7], Proposition 3.2, there exists a morphism

$$
D_{X_{(r)} I}^{\log } \longrightarrow X_{\left(r-I^{\#}+1\right)}^{\log } \times_{K} \overline{\mathcal{M}}_{0, I^{\#}+1}^{\log }
$$


which is of type $\mathbb{N}$; moreover, these morphisms fit into a commutative diagram

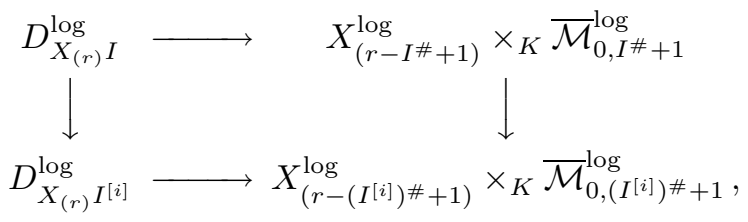

where the left-hand vertical arrow is the morphism induced by the composite $p_{X_{(r-1):} \text { log }}^{\log } \delta_{X_{(r)} I}^{\log }$, and if $i \notin I$ (respectively, $i \in I$ ), then the right-hand vertical arrow is the morphism obtained as the base-change of the morphism $p_{X_{(r-I \#)}^{\log }: i^{\prime}}: X_{\left(r-I^{\#}+1\right)}^{\log } \rightarrow X_{\left(r-\left(I^{[i]}\right) \#+1\right)}^{\log }=X_{\left(r-I^{\#}\right)}^{\log }\left(\right.$ respectively, $\overline{\mathcal{M}}_{0, I^{\#}+1}^{\log } \rightarrow$ $\overline{\mathcal{M}}_{0,\left(I^{[i]}\right) \#+1}^{\log }=\overline{\mathcal{M}}_{0, I \#}^{\log }$ obtained by forgetting the $i^{\prime}$-th section), where $i^{\prime}$ is the integer such that $\{1,2, \cdots, r\} \backslash I=\left\{i_{1}, i_{2}, \cdots, i_{r-I \#}\right\} ; i_{1}<i_{2}<\cdots<i_{r-I \#}$; $i=i_{i^{\prime}}$ (respectively, $\left.I=\left\{i_{1}, i_{2}, \cdots, i_{I^{\#}}\right\} ; i_{1}<i_{2}<\cdots<i_{I^{\#}} ; i=i_{i^{\prime}}\right)$. Now it follows from [6], Proposition 4; Remark 15; [7], Lemma 4.2, (iii), that the above diagram induces a commutative diagram

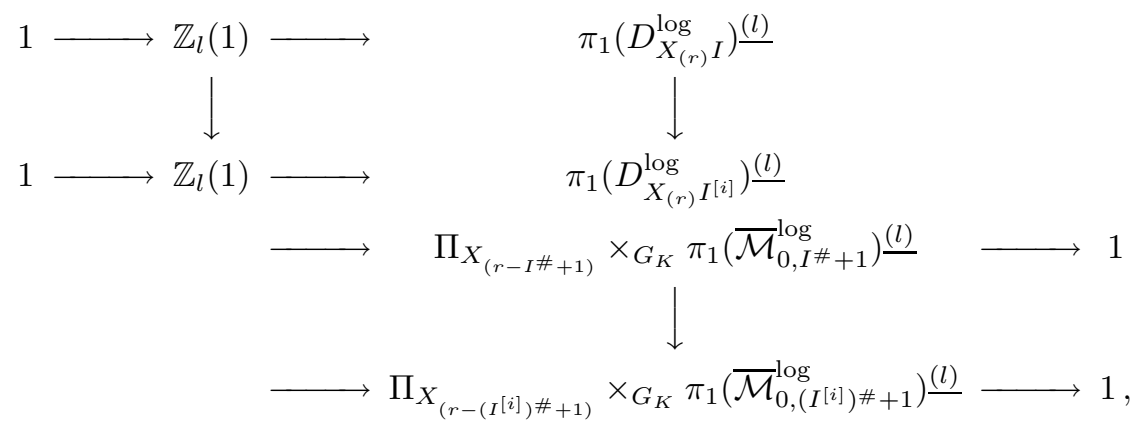

where " $\pi_{1}\left(\overline{\mathcal{M}}_{0,(-)}^{\log }\right) \stackrel{(l)}{ }$ " is the geometrically pro-l log fundamental group of " $\overline{\mathcal{M}}_{0,(-)}^{\log }$ ", and the horizontal sequences are exact; moreover, by considering the restriction of $D_{X_{(r)} I}^{\log } \rightarrow D_{X_{(r)} I^{[i]}}^{\log }$ to the generic point of $D_{X_{(r)} I}^{\log }$, the lefthand vertical arrow is an isomorphism. Thus, if $i \notin I$ (respectively, $i \in I$ ), then the kernel of the morphism $\pi_{1}\left(D_{X_{(r)} I}^{\log }\right) \stackrel{(l)}{\rightarrow} \pi_{1}\left(D_{X_{(r)} I^{[i]}}^{\log }\right) \stackrel{(l)}{\text { (in the above }}$

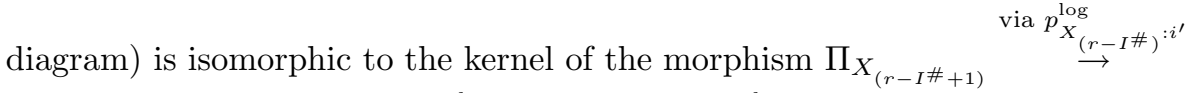

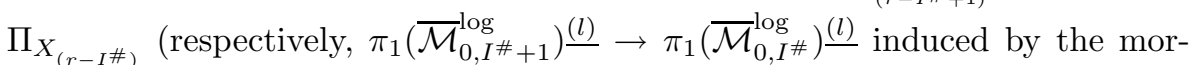
phism $\overline{\mathcal{M}}_{0, I}^{\log } \rightarrow \overline{\mathcal{M}}_{0, I}^{\log }$ obtained by forgetting the $i^{\prime}$-th section). Now the fiber of the morphism $\overline{\mathcal{M}}_{0, I^{\#}+1}^{\log } \rightarrow \overline{\mathcal{M}}_{0, I^{\#}}^{\log }$ (obtained by forgetting the $i^{\prime}$-th section) at a geometric point of Spec $K^{\text {sep }} \rightarrow \overline{\mathcal{M}}_{0, I \#}^{\log }$ whose image lies in the 
interior of $\overline{\mathcal{M}}_{0, I^{\#}}^{\log }$ is isomorphic to the log scheme obtained by equipping $\mathbb{P}_{K^{\text {sep }}}^{1}$ with the $\log$ structure associated to the reduced divisor consisting of distinct $I^{\#}$ elements of $\mathbb{P}_{K \text { sep }}^{1}\left(K^{\text {sep }}\right)$; thus, if $i \in I$, then the kernel of the morphism

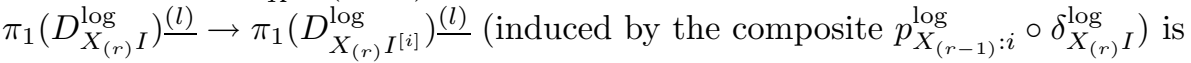
the free profinite group of $\operatorname{rank} I^{\#}-1$. More precisely, if we denote by $\Delta_{\mathbb{P} \backslash I \#}$ the pro- $l$ fundamental group of the log scheme obtained by equipping $\mathbb{P}_{K^{\text {sep }}}^{1}$ with the $\log$ structure associated to the reduced divisor consisting of distinct $I^{\#}$ elements of $\mathbb{P}_{K^{\text {sep }}}^{1}\left(K^{\mathrm{sep}}\right)$, then the kernel of $\pi_{1}\left(D_{X_{(r)} I}^{\log }\right) \frac{(l)}{\rightarrow} \pi_{1}\left(D_{X_{(r)} I^{[i]}}^{\log }\right) \frac{(l)}{\text { is }}$ naturally isomorphic to $\Delta_{\mathbb{P} \backslash I \#}$; moreover, by base-changing the exact sequence

$$
1 \longrightarrow \mathbb{Z}_{l}(1) \longrightarrow \pi_{1}\left(D_{X_{(r)} I}^{\log }\right) \stackrel{(l)}{\longrightarrow} \Pi_{X_{(r-I \#+1)}} \times_{G_{K}} \pi_{1}\left(\overline{\mathcal{M}}_{0, I \#}^{\log }\right) \stackrel{(l)}{\longrightarrow} 1
$$

via the natural inclusion

$$
\Delta_{\mathbb{P} \backslash I^{\#}} \stackrel{\sim}{\rightarrow}\{1\} \times_{\{1\}} \Delta_{\mathbb{P} \backslash I^{\#}} \hookrightarrow \Pi_{X_{(r-I}^{\#+1)}} \times_{G_{K}} \pi_{1}\left(\overline{\mathcal{M}}_{0, I^{\#}+1}^{\log }\right) \frac{(l)}{,}
$$

we obtain an exact sequence

$$
1 \longrightarrow \mathbb{Z}_{l}(1) \longrightarrow \mathfrak{P}_{X_{(r)} I} \longrightarrow \Delta_{\mathbb{P} \backslash I^{\#}} \longrightarrow 1,
$$

where

$$
\mathfrak{P}_{X_{(r)} I} \stackrel{\text { def }}{=} \pi_{1}\left(D_{X_{(r)} I}^{\log }\right) \stackrel{(l)}{\left.\times_{\left(\Pi_{X}(r-I \#+1)\right.} \times_{G_{K}} \pi_{1}\left(\overline{\mathcal{M}}_{0, I}^{\log }\right) \stackrel{(l)}{ }\right)} \Delta_{\mathbb{P} \backslash I \#} .
$$

Now by considering the kernel of the morphism $\pi_{1}\left(D_{X_{(r)} I}^{\log } \stackrel{(l)}{\rightarrow} \pi_{1}\left(D_{X_{(r)} I^{[i]}}^{\log }\right) \frac{(l)}{2}\right.$ (induced by the composite $p_{X_{(r-1): i}}^{\log } \circ \delta_{X_{(r)} I}^{\log }$ ), we obtain a section

$$
\Delta_{\mathbb{P} \backslash I} \longrightarrow \mathfrak{P}_{X_{(r)} I}
$$

of the above exact sequence. We shall refer to this section $\Delta_{\mathbb{P} \backslash I \#} \rightarrow \mathfrak{P}_{X_{(r)} I}$ of the above exact sequence as the section of $\mathfrak{P}_{X_{(r)} I} \rightarrow \Delta_{\mathbb{P} \backslash I \#}$ induced by $p_{X_{(r-1)}: i}^{\log }$.

Definition 2.3. Let $r \geq 2$ be an integer, and $I$ a subset of $\{1,2, \cdots, r\}$ of cardinality $I^{\#} \geq 2$. Then we shall denote by $\mathfrak{I}_{X_{(r)} I}$ the kernel of the surjection

$$
\mathfrak{D}_{X_{(r)} I} \rightarrow \Pi_{X_{(r-I}^{\#+1)}} \times_{G_{K}} \pi_{1}\left(\overline{\mathcal{M}}_{0, I \#+1}^{\log }\right) \underline{(l)}
$$

obtained in the above argument. (Note that this subgroup is well-defined, up to conjugation.) By the above argument, $\mathfrak{I}_{X_{(r+1)}\{i, j\}}$ is the inertia subgroup (well-defined, up to conjugation) associated to the cusp (of the geometric fiber of $p_{U_{X_{(r)}}: i}: U_{X_{(r+1)}} \rightarrow U_{X_{(r)}}$ at a geometric point of $U_{X_{(r)}}$ ) determined by the divisor $D_{X_{\underline{(r+1)}}}\{i, j\} \subseteq X_{\underline{(r+1)}}$. 
Lemma 2.1. In the above situation, the images via the section of $\mathfrak{P}_{X_{(r)} I}$ $\rightarrow \Delta_{\mathbb{P} \backslash I \#}$ induced by $p_{X_{(r-1)}: i}^{\log }$ of the $\left(I^{\#}-1\right)$ inertia subgroups of $\Delta_{\mathbb{P} \backslash I \#}$ (welldefined, up to conjugation in $\left.\Delta_{\mathbb{P} \backslash I^{\#}}\right)$ corresponding to inertia subgroups associated to the cusps (of a geometric fiber $\overline{\mathcal{M}}_{0, I^{\#+1}}^{\log } \rightarrow \overline{\mathcal{M}}_{0, I^{\#}}^{\log }$ obtained by forgetting the $i^{\prime}$-th section) determined by the first $\left(I^{\#}-1\right)$ marked points of $\overline{\mathcal{M}}_{0, I^{\#}+1}^{\log } \rightarrow \overline{\mathcal{M}}_{0, I \#}^{\log }$ are conjugates of $\mathfrak{I}_{X_{(r+1)}\{i, j\}}$ in $\Delta_{X_{(r)}}$, where $j \in I$.

Proof. Let $\bar{x}^{\log } \rightarrow D_{X_{(r-1)} I^{[i]}}^{\log }$ be a strict geometric point of $D_{X_{(r-1)} I^{[i]}}^{\log }$ (i.e., a strict morphism whose underlying morphism is a geometric point [cf. [6], Definition 1, (i)]) whose image of the underlying morphism of schemes is the generic point. First, we consider the log structure of $D_{X_{(r) I}}^{\log } \times{ }_{D_{X_{(r-1)} I^{[i]}}^{\log }} \bar{x}^{\log }$ (where the morphism $D_{X_{(r)} I}^{\log } \rightarrow D_{X_{(r-1)} I^{[i]}}^{\log }$ is the morphism induced by $\left.p_{X_{(r-1):}}^{\log } \circ \delta_{X_{(r)} I}^{\log }\right)$ and $\bar{x}^{\log }$. It is immediate that the log structure of $\bar{x}^{\log }$ has the chart:

$$
\begin{aligned}
& \mathbb{N} \longrightarrow k(\bar{x}) \\
& n \mapsto 0^{n} .
\end{aligned}
$$

By the definitions, the underlying scheme of $D_{X_{(r)} I}^{\log } \times_{D_{X_{(r-1)} I^{[i]}}^{\log }} \bar{x}^{\log }$ is the projective line $\mathbb{P} \frac{1}{x}$ over $\bar{x}$, and the $\log$ structure of $D_{X_{(r)} I}^{\log } \times_{D_{X_{(r-1)} I^{[i]}}^{\log }} \bar{x}^{\log }$ has the following chart:

Let $\bar{y} \rightarrow \mathbb{P} \frac{1}{\bar{x}}$ be a geometric point of the underlying scheme $D_{X_{(r)} I}$ $\times_{D_{\left.X_{(r-1)}{ }^{[i]}\right]}} \bar{x}\left(\simeq \mathbb{P} \frac{1}{x}\right)$ of $D_{X_{(r)} I}^{\log } \times_{D_{X_{(r-1)} I^{[i]}}^{\log }} \bar{x}^{\log }$. Then the following hold:

(1) If the image of $\bar{y} \rightarrow \mathbb{P} \frac{1}{x}$ does not lie on the $D_{X_{(r)}\{i, j\}}^{\log }$ 's (where $j \in I)$, then the $\log$ structure of $D_{X_{(r)} I}^{\log } \times_{D_{X_{(r-1)} I^{[i]}}^{\log }} \bar{x}^{\log }$ at $\bar{y} \rightarrow \mathbb{P} \frac{1}{x}$ is induced by

$$
\begin{aligned}
& \mathbb{N} \longrightarrow k(\bar{y})[[t]] \\
& n \mapsto 0^{n} .
\end{aligned}
$$

Moreover, the projection $D_{X_{(r)} I}^{\log } \times_{D_{X_{(r-1)} I^{[i]}}^{\log }} \bar{x}^{\log } \rightarrow \bar{x}^{\log }$ has the chart:

$$
\begin{array}{ccc}
k(\bar{x}) \longrightarrow & \longrightarrow(\bar{y})[[t]] \\
\uparrow & \uparrow \\
\mathbb{N} & \stackrel{\operatorname{id}_{\mathbb{N}}}{\longrightarrow} & \mathbb{N} .
\end{array}
$$


(2) If the image of $\bar{y} \rightarrow \mathbb{P} \frac{1}{x}$ lies in $D_{X_{(r)}}^{\log }\{i, j\}$ (where $j \in I$ ), then the $\log$ structure of $D_{X_{(r)} I}^{\log } \times{ }_{D_{X_{(r-1)} I^{[i]}}^{\log }} \bar{x}^{\log }$ at $\bar{y} \rightarrow \mathbb{P} \frac{1}{x}$ is induced by

$$
\begin{aligned}
\mathbb{N}^{\oplus 2} & \longrightarrow k(\bar{y})[[t]] \\
(n, m) & \mapsto 0^{n} \cdot t^{m} .
\end{aligned}
$$

Moreover, the projection $D_{X_{(r)} I^{\log }}{ }_{D_{X_{(r-1)} I^{[i]}}^{\log }} \bar{x}^{\log } \rightarrow \bar{x}^{\log }$ has the chart:

$$
\begin{array}{ccc}
k(\bar{x}) & \longrightarrow & k(\bar{y})[[t]] \\
\uparrow & & \uparrow \\
\mathbb{N} & \longrightarrow & \mathbb{N}^{\oplus 2} \\
n & \mapsto & (n, 0) .
\end{array}
$$

(3) If the image of $\bar{y} \rightarrow \mathbb{P}_{\bar{x}}^{1}$ lies in $D_{X_{(r)} J}^{\log }$ (where $J$ is the subset of $\{1,2, \cdots, r\}$ which is uniquely determined by the condition that $J \subsetneq I$ and $\left.J^{[i]}=I^{[i]}\right)$, then the log structure of $D_{X_{(r)} I}^{\log } \times{ }_{D_{X_{(r-1)}}^{\log }}$ $\bar{x}^{\log }$ at $\bar{y} \rightarrow \mathbb{P}_{\bar{x}}^{1}$ is induced by

$$
\begin{aligned}
\mathbb{N}^{\oplus 2} & \longrightarrow k(\bar{y})[[t]] \\
(n, m) & \mapsto 0^{n} \cdot t^{m} .
\end{aligned}
$$

Moreover, the projection $D_{X_{(r)} I^{\log }}{ }_{D_{X_{(r-1)} I^{[i]}}^{\log }} \bar{x}^{\log } \rightarrow \bar{x}^{\log }$ has the chart:

$$
\begin{array}{ccc}
k(\bar{x}) & \longrightarrow & k(\bar{y})[[t]] \\
\uparrow & & \uparrow \\
\mathbb{N} & \longrightarrow & \mathbb{N}^{\oplus 2} \\
n & \mapsto & (n, n) .
\end{array}
$$

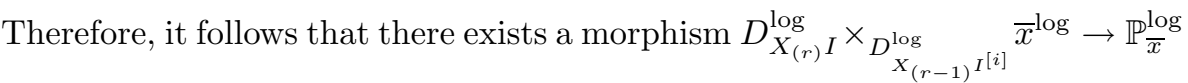
which is of type $\mathbb{N}$ (where $\mathbb{P}_{\bar{x}}^{\text {log }}$ is the log scheme obtained by equipping $\mathbb{P} \frac{1}{x}$ with the $\log$ structure associated to the divisor determined by the divisors " $D_{X_{(r)} I}^{\log } \cap D_{X_{(r)}\{i, j\}}^{\log }$ " [where $\left.j \in I\right]$ and " $D_{X_{(r)} I}^{\log } \cap D_{X_{(r)} J}^{\log }$ " [where $J$ is as in (3)]) which fits into a natural commutative diagram:

$$
\begin{aligned}
& D_{X_{(r)} I}^{\log } \times{ }_{D_{X_{(r-1)} I^{[i]}}^{\log }} \bar{x}^{\log } \longrightarrow \mathbb{P}_{\bar{x}}^{\log } \\
& \begin{array}{cl}
\operatorname{pr}_{2} \downarrow & \downarrow \\
\bar{x}^{\log } \longrightarrow \bar{x} .
\end{array}
\end{aligned}
$$


(Note that by (3), this diagram is not cartesian.) This diagram induces a commutative diagram

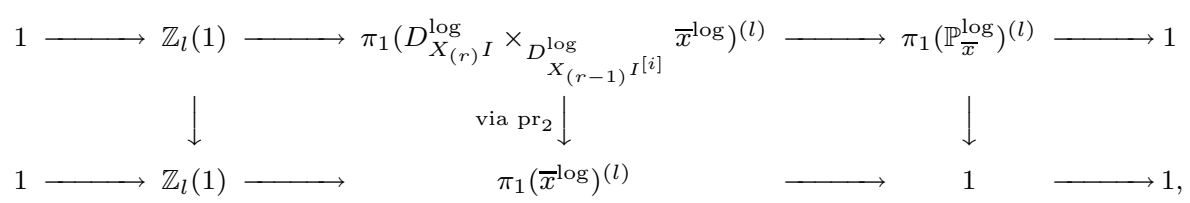

where the horizontal sequences are exact (cf. [6], Proposition 4; Remark 15; [7], Lemma 4.2, (iii)). By (1), the left-hand vertical arrow is an isomorphism, i.e., the right-hand square is cartesian. Thus, since the kernel of the middle vertical arrow $\pi_{1}\left(D_{X_{(r)} I}^{\log } \times{ }_{D_{X_{(r-1)} I^{[i]}}^{\log }} \bar{x}^{\log }\right)^{(l)} \stackrel{\text { via pr }}{\rightarrow} \pi_{1}\left(\bar{x}^{\log }\right)^{(l)}$ is naturally isomorphic to the kernel of $\pi_{1}\left(D_{X_{(r)} I}^{\log }\right) \stackrel{(l)}{\rightarrow} \pi_{1}\left(D_{X_{(r)} I^{[i]}}^{\log }\right) \frac{(l)}{}$, we conclude that the kernel of

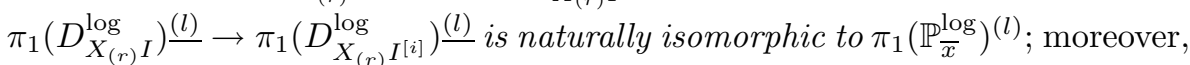
it follows from the definitions that this isomorphism determines the section of $\mathfrak{P}_{X_{(r)} I} \rightarrow \Delta_{\mathbb{P} \backslash I}^{\#}\left(\simeq \pi_{1}\left(\mathbb{P}_{\bar{x}}^{\log }\right)^{(l)}\right)$ induced by $p_{X_{(r-1)}: i}^{\log }$. Thus, Lemma 2.1 follows immediately from observations (2) and (3).

Proposition 2.4 (cf. [7], Theorem 4.1). $\quad$ Let $r \geq 2$ be an integer. Then conjugates in $\Delta_{X_{(r+1)}}$ of the subgroups

$$
\mathfrak{D}_{X_{(r+1)}\{1,2\}}^{\Delta} ; \mathfrak{D}_{X_{(r+1)}\{2,3\}}^{\Delta} \subseteq \Delta_{X_{(r+1)}}
$$

topologically generate $\Delta_{X_{(r+1)}}$.

Proof. Since the composite

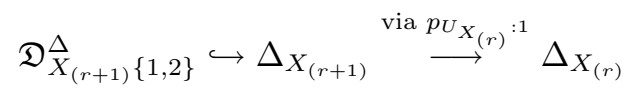

is surjective, it is enough to show that the subgroup topologically generated by the subgroups in question includes the kernel of the morphism $\Delta_{X_{(r+1)}} \rightarrow \Delta_{X_{(r)}}$ induced by $p_{U_{X_{(r)}}: 1}$, i.e., $\Delta_{X_{(r+1)} / X_{(r)}}^{(1)}$. On the other hand, if $\bar{x}^{\log } \rightarrow X_{(r)}^{\log }$ is a strict geometric point whose image is the generic point of the divisor $D_{X_{(r)}}^{\log }\{1,2\}$ of $X_{(r)}^{\log }$, then by [7], Proposition 2.2, the image of

$$
\stackrel{\lim }{\longleftarrow} \pi_{1}\left(X_{(r+1)}^{\log } \times_{X_{(r)}^{\log }} \bar{x}_{\lambda}^{\log }\right)^{(l)} \stackrel{\text { via pr }_{1}}{\longrightarrow} \Delta_{X_{(r+1)}}
$$

(where the projective limit is over all reduced covering points $\bar{x}_{\lambda}^{\log } \rightarrow \bar{x}^{\log }$ of $\bar{x}^{\log }$ [cf. [6], Definition 1, (ii)]) is $\Delta_{X_{(r+1)} / X_{(r)}}^{(1)}$. Moreover, since the irreducible components of the underlying scheme of $X_{(r+1)}^{\log } \times X_{(r)}^{\log } \bar{x}_{\lambda}^{\log }\left(=X_{(r+1)}^{\log } \times_{D_{X_{(r)}\{1,2\}}^{\log }} \bar{x}_{\lambda}^{\log }\right)$ 
are the underlying schemes of $D_{X_{(r+1)}\{2,3\}}^{\log } \times_{D_{X_{(r)}}^{\log }{ }_{11,2\}}} \bar{x}_{\lambda}^{\log }$ and $D_{X_{(r+1)}\{1,2,3\}}^{\log }$ ${ }_{D_{X(r)}^{\log }\{1,2\}} \bar{x}_{\lambda}^{\log }$ (cf. [7], Proposition 3.1, (v)), by the evident logarithmic version of [22], Corollary 2.3.3 (cf. the proof of [22], Lemma 6.2.7), the group

$$
\lim _{1}\left(X_{(r+1)}^{\log } \times_{X_{(r)}^{\log }} \bar{x}_{\lambda}^{\log }\right)^{(l)}
$$

is topologically generated by the images of the natural morphisms from

$$
\underset{\lim }{\longleftarrow} \pi_{1}\left(D_{X_{(r+1)}\{2,3\}}^{\log } \times{ }_{D_{X}^{\log }\{1,2\}} \bar{x}_{\lambda}^{\log }\right)^{(l)}
$$

and

$$
\underset{\lim }{\longleftarrow} \pi_{1}\left(D_{X_{(r+1)}\{1,2,3\}}^{\log } \times_{D_{X_{(r)}\{1,2\}}^{\log }} \bar{x}_{\lambda}^{\log )^{(l)}} .\right.
$$

Thus, it is enough to show that the subgroup topologically generated by the subgroups in question includes the image of the natural morphisms from

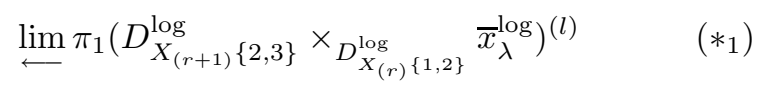

and

$$
\underset{\lim }{\longleftarrow} \pi_{1}\left(D_{X_{(r+1)}}^{\log }\{1,2,3\} \times_{D_{X_{(r)}}^{\log }\{1,2\}} \bar{x}_{\lambda}^{\log }\right)^{(l)} \quad\left(*_{2}\right) .
$$

Now since it is immediate that the natural strict morphism $D_{X_{(r+1)}}^{\log }\{2,3\}$ $\times_{D_{X(r)}^{\log \{1,2\}}} \bar{x}^{\log } \rightarrow X_{(r+1)}^{\log }$ factors through $D_{X_{(r+1)}\{2,3\}}^{\log }$, it thus follows that the image of the first group $\left(*_{1}\right)$ is included in a conjugate of $\mathfrak{D}_{X_{(r+1)}}^{\Delta}\{2,3\}$. On the other hand, it follows immediately from Lemma 2.1 (together with observation (3) in the proof of Lemma 2.1) that the image of the second group $\left(*_{2}\right)$ is included in the subgroup topologically generated by conjugates of the kernel of the composite

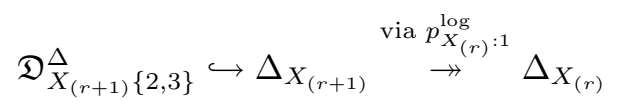

and $\mathfrak{I}_{X_{(r+1)}\{1,2\}}$. This completes the proof of Proposition 2.4 .

Lemma 2.2. Let $r \geq 2$ and $1 \leq i<j \leq r$ be integers. Then the subgroup $\mathfrak{D}_{X_{(r)}\{i, j\}}$ (respectively, $\mathfrak{D}_{X_{(r)}\{i, j\}}^{\Delta}$ ) of $\Pi_{X_{(r)}}$ (respectively, $\Delta_{X_{(r)}}$ ) is the normalizer (where we refer to the discussion entitled "Groups" in Introduction concerning the term "normalizer") of $\mathfrak{I}_{X_{(r)}\{i, j\}}$ in $\Pi_{X_{(r)}}$ (respectively, $\Delta_{X_{(r)}}$ ). 
Proof. Since $\mathfrak{I}_{X_{(r)}\{i, j\}}$ is normal in $\mathfrak{D}_{X_{(r)}\{i, j\}}$ (respectively, $\mathfrak{D}_{X_{(r)}\{i, j\}}^{\Delta}$ ), the normalizer of $\mathfrak{I}_{X_{(r)}\{i, j\}}$ includes $\mathfrak{D}_{X_{(r)}\{i, j\}}$ (respectively, $\mathfrak{D}_{X_{(r)}\{i, j\}}^{\Delta}$. Moreover, we have a commutative diagram:

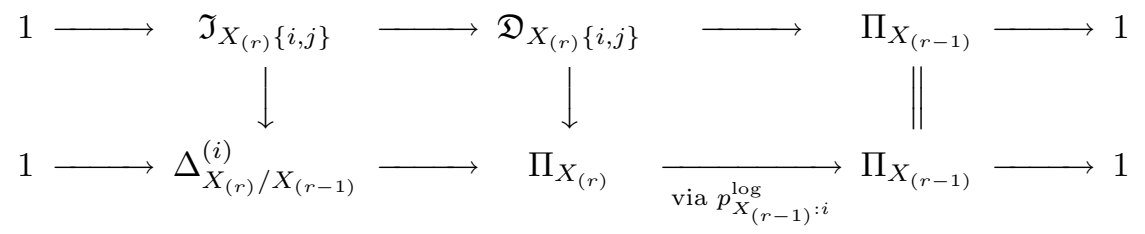

(respectively,

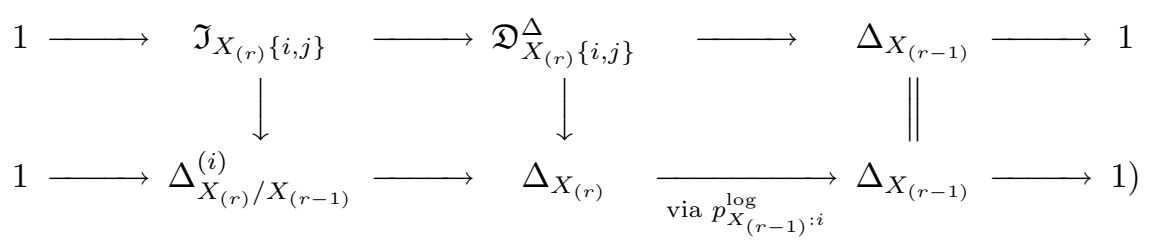

(cf. the second diagram in the discussion following Proposition 2.3, also Definition 2.3). Therefore, it is enough to show that the normalizer of $\mathfrak{I}_{X_{(r)}\{i, j\}}$ in $\Delta_{X_{(r)} / X_{(r-1)}}^{(i)}$ is $\mathfrak{I}_{X_{(r)}\{i, j\}}$. On the other hand, this is well-known (cf. e.g., [19], $(2.3 .1))$.

Remark 4. By a similar argument to the argument used in the proof of Lemma 2.2 (by replacing [19], (2.3.1) by [13], Lemma 1.3.12), we conclude that:

Let $r \geq 2$ and $1 \leq i<j \leq r$ be integers. Then the subgroup $\mathfrak{D}_{X_{(r)}\{i, j\}}$ (respectively, $\mathfrak{D}_{X_{(r)}\{i, j\}}^{\Delta}$ ) of $\Pi_{X_{(r)}}$ (respectively, $\Delta_{X_{(r)}}$ ) is the commensurator (where we refer to the discussion entitled "Groups" in Introduction concerning the term "commensurator") of $\mathfrak{I}_{X_{(r)}\{i, j\}}$ in $\Pi_{X_{(r)}}$ (respectively, $\Delta_{X_{(r)}}$ ).

Definition 2.4. Let $r \geq 2$ and $1 \leq i<j \leq r$ be integers.

(i) We shall denote by $U_{X_{(r)} \underline{\{i, j\}}}$ the fiber product of

$$
\begin{aligned}
& U_{X_{(r-1)}} \\
& \downarrow^{p_{U_{(r-2)}}}{ }^{: j-1} \\
& U_{X_{(r-1)}} \underset{p_{U_{X_{(r-2)}}: i}}{\longrightarrow} U_{X_{(r-2)}} \text {. }
\end{aligned}
$$

Moreover, we shall denote by $p_{U \frac{\{i, j\}}{X_{(r-1)}}: i}$ and $p_{U \frac{\{i, j\}}{X_{(r-1)}}: j}$ the projections $U_{X_{(r)} \underline{\{i, j\}}} \rightarrow U_{X_{(r-1)}}$ such that $p_{U_{X_{(r-2)}}: j-1} \circ p_{U_{X_{(r-1)}}: i}=p_{U_{X_{(r-2)}}: i} \circ p_{U_{X_{(r-1)}}^{\{i, j\}}}$. 
(ii) By the definition of $U_{X_{(r)} \underline{\{i, j\}}}$, the commutative diagram

$$
\begin{aligned}
U_{X_{(r)}} & \stackrel{p_{U_{X_{(r-1)}}: i}^{\longrightarrow}}{\longrightarrow} U_{X_{(r-1)}} \\
p_{U_{X_{(r-1)}}: j} \downarrow & \downarrow^{p_{X_{(r-2)}}: j-1} \\
U_{X_{(r-1)}} & \underset{p_{X_{(r-2)}: i}}{\longrightarrow} U_{X_{(r-2)}}
\end{aligned}
$$

induces a morphism $U_{X_{(r)}} \rightarrow U_{X_{(r)}\{i, j\}}$. We shall denote this morphism by $\iota_{U_{X_{(r)}}\{i, j\}}$. By the definition of $\iota_{U_{X_{(r)}}\{i, j\}}$, it is immediate that $\iota_{U_{X_{(r)}} \underline{\{i, j\}}}$ : $U_{X_{(r)}} \rightarrow U_{X_{(r)}\{i, j\}}$ is an open immersion, which is a "partial compactification", i.e., the natural open immersion $U_{X_{(r)}} \hookrightarrow X_{(r)}$ factors through $\iota_{U_{X_{(r)}} \underline{\{i, j\}}} ;$ moreover,

$$
U_{X_{(r)} \underline{\{i, j\}}}=X_{\underline{(r)}} \backslash \bigcup_{\left\{i^{\prime}, j^{\prime}\right\} \neq\{i, j\}} D_{X_{\underline{(r)}\left\{i^{\prime}, j^{\prime}\right\}}} .
$$

(iii) We shall denote by $\Pi_{X_{(r)} \underline{\{i, j\}}}$ the geometrically pro-l fundamental group of $U_{X_{(r)} \underline{\{i, j\}}}$, and by $\Delta_{X_{(r)}} \underline{\{i, j\}}$ the kernel of the natural surjection

$$
\Pi_{X_{(r)} \underline{\{i, j\}}} \rightarrow G_{K} .
$$

Thus, we have an exact sequence

$$
1 \longrightarrow \Delta_{X_{(r)} \underline{\{i, j\}}} \longrightarrow \Pi_{X_{(r)} \underline{\{i, j\}}} \longrightarrow G_{K} \longrightarrow 1 \text {. }
$$

Lemma 2.3. Let $r \geq 2$ and $1 \leq i<j \leq r$ be integers. Then the following diagram induced by the cartesian diagram which appears in the definition of $U_{X_{(r)}\{i, j\}}$ is cartesian:

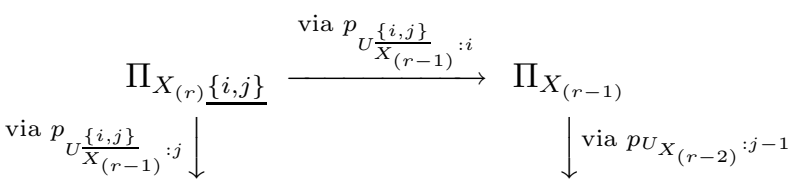

$$
\begin{aligned}
& \Pi_{X_{(r-1)}} \underset{\text { via } p_{U_{X}(r-2)}: i}{\longrightarrow} \Pi_{X_{(r-2)}} .
\end{aligned}
$$

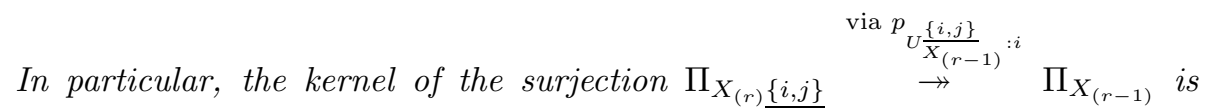
naturally isomorphic to $\Delta_{X_{(r-1)} / X_{(r-2)}}^{(i)}$. 
Proof. This follows immediately from the fact that the sequence obtained as the base-change of

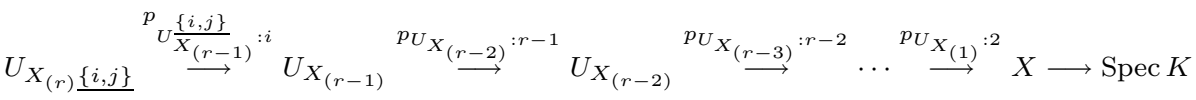

from $K$ to $\bar{K}$ is a successive extension of hyperbolic curves of product type (cf. Definition 1.4, (i)), together with Proposition 1.4, (i).

In the following, assume that

the field $K$ is a finite field.

Let us denote by $p_{K}$ (respectively, $q_{K}$ ) the characteristic (respectively, cardinality) of $K$. We shall fix an algebraic closure $\bar{K}$ of $K$. We shall denote by $G_{K}$ the Galois group of $\bar{K}$ over $K$, and by $\operatorname{Fr}_{K} \in G_{K}$ the Frobenius element of $G_{K}$. Moreover, let $L$ be a finite field whose characteristic (respectively, cardinality) we denote by $p_{L}$ (respectively, $q_{L}$ ) such that $l$ is invertible in $L$ (i.e., $\left.l \neq p_{L}\right), \bar{L}$ an algebraic closure of $L, G_{L} \stackrel{\text { def }}{=} \operatorname{Gal}(\bar{L} / L), Y$ a proper hyperbolic curve over $L$, and $\alpha_{(r)}: \Pi_{X_{(r)}} \stackrel{\sim}{\rightarrow} \Pi_{Y_{(r)}}$ an isomorphism. Then it follows from the "Riemann hypothesis for abelian varieties over finite fields" (cf. e.g., [18], p. 206) and the fact that $\mathbb{Z}_{l}(1)$ (where "(1)" denotes a Tate twist) is "of weight 2 " (since the eigenvalues of the action of " $\operatorname{Fr}_{(-)}$" are " $q_{(-)}$") that the quotient $\Pi_{X_{(r)}} \rightarrow G_{K}$ (respectively, $\Pi_{Y_{(r)}} \rightarrow G_{L}$ ) arising from the structure morphism $U_{X_{(r)}} \rightarrow \operatorname{Spec} K$ (respectively, $U_{Y_{(r)}} \rightarrow$ Spec $L$ ) may be characterized as the (unique) maximal ( $\widehat{\mathbb{Z}}$-) free abelian quotient of $\Pi_{X_{(r)}}$ (respectively, $\Pi_{Y_{(r)}}$ ). Therefore, the isomorphism $\alpha_{(r)}$ induces an isomorphism $\alpha_{(0)}: G_{K} \rightarrow G_{L}$.

Remark 5. As was pointed out to the author by the referee, the above group-theoretic reconstruction of $G_{K}$ from $\Pi_{X_{(r)}}$ (strictly speaking, the fact that the image of the composite $\Delta_{X_{(r)}} \hookrightarrow \Pi_{X_{(r)}} \rightarrow \Pi_{X_{(r)}}^{\mathrm{ab}}$ is torsion) can also be deduced from geometric class field theory.

Definition 2.5 (cf. [16], Definition 1.5, (iii)). We shall say that an isomorphism $\alpha_{(r)}: \Pi_{X_{(r)}} \stackrel{\sim}{\rightarrow} \Pi_{Y_{(r)}}$ is Frobenius-preserving if the isomorphism $\alpha_{(0)}: G_{K} \rightarrow G_{L}$ obtained as above maps the Frobenius element of $G_{K}$ to the Frobenius element of $G_{L}$. Note that in general, an isomorphism of geometrically pro- $l$ fundamental groups is not Frobenius-preserving (cf. Remark 10, (ii) below).

Proposition 2.5. $\quad$ Let $\alpha_{(r)}: \Pi_{X_{(r)}} \stackrel{\sim}{\rightarrow} \Pi_{Y_{(r)}}$ be an isomorphism. Then the following hold: 
(i) There exists an element $\sigma$ of the symmetric group on $r$ letters such that for any integer $1 \leq i \leq r$, the isomorphism $\alpha_{(r)}$ maps the kernel $\Delta_{X_{(r)} / X_{(r-1)}}^{(i)}$ of the surjection $\Pi_{X_{(r)}} \rightarrow \Pi_{X_{(r-1)}}$ induced by $p_{X_{(r-1)}: i}^{\log }$ bijectively onto the kernel $\Delta_{Y_{(r)} / Y_{(r-1)}}^{(\sigma(i))}$ of the surjection $\Pi_{Y_{(r)}} \rightarrow \Pi_{Y_{(r-1)}}$ induced by $p_{Y_{(r-1)}: \sigma(i)}^{\log }$.

(ii) Assume, moreover, that $\alpha_{(r)}: \Pi_{X_{(r)}} \stackrel{\sim}{\rightarrow} \Pi_{Y_{(r)}}$ is a Frobenius-preserving isomorphism (cf. Definition 2.5). Then, for a section $G_{K} \rightarrow \Pi_{X_{(r)}}$ of the natural morphism $\Pi_{X_{(r)}} \rightarrow G_{K}$, this section arises from a K-rational point of $U_{X_{(r)}}$ if and only if the section of the natural morphism $\Pi_{Y_{(r)}} \rightarrow$ $G_{L}$ corresponding to the section $G_{K} \rightarrow \Pi_{X_{(r)}}$ under the isomorphism $\alpha_{(r)}$ arises from an L-rational point of $U_{Y_{(r)}}$.

(iii) Assume, moreover, that $r \geq 2$. Then, for any integers $1 \leq i<j \leq r$, the isomorphism $\alpha_{(r)}$ maps $\mathfrak{I}_{X_{(r)}\{i, j\}}$ (respectively, $\mathfrak{D}_{X_{(r)}\{i, j\}}$ ) bijectively onto a conjugate of $\mathfrak{I}_{Y_{(r)}\{\sigma(i), \sigma(j)\}}$ (respectively, $\mathfrak{D}_{Y_{(r)}\{\sigma(i), \sigma(j)\}}$ ) by an element of the kernel $\Delta_{Y_{(r)}}$ of the natural surjection $\Pi_{Y_{(r)}} \rightarrow G_{L}$, where $\sigma$ is the element of the symmetric group on $r$ letters defined in (i).

(iv) Under the assumption in the statement of (iii), for any integers $1 \leq i<$ $j \leq r$, let us denote by

$$
\tau_{X_{(r-1)}\{i, j\}}: \Pi_{X_{(r)}} / \Delta_{X_{(r)} / X_{(r-1)}}^{(i)} \stackrel{\sim}{\longrightarrow} \Pi_{X_{(r)}} / \Delta_{X_{(r)} / X_{(r-1)}}^{(j)}
$$

(respectively,

$$
\left.\tau_{Y_{(r-1)}}\{i, j\}: \Pi_{Y_{(r)}} / \Delta_{Y_{(r)} / Y_{(r-1)}}^{(i)} \stackrel{\sim}{\longrightarrow} \Pi_{Y_{(r)}} / \Delta_{Y_{(r)} / Y_{(r-1)}}^{(j)}\right)
$$

the isomorphism obtained as the composite

$$
\Pi_{X_{(r)}} / \Delta_{X_{(r)} / X_{(r-1)}}^{(i)} \stackrel{\sim}{\longrightarrow} \Pi_{X_{(r-1)}} \stackrel{\sim}{\longleftarrow} \Pi_{X_{(r)}} / \Delta_{X_{(r)} / X_{(r-1)}}^{(j)}
$$

(respectively,

$$
\left.\Pi_{Y_{(r)}} / \Delta_{Y_{(r)} / Y_{(r-1)}}^{(i)} \stackrel{\sim}{\longrightarrow} \Pi_{Y_{(r-1)}} \stackrel{\sim}{\longleftarrow} \Pi_{Y_{(r)}} / \Delta_{Y_{(r)} / Y_{(r-1)}}^{(j)}\right) .
$$

Then the following diagram commutes:

$$
\begin{aligned}
& \Pi_{X_{(r)}} / \Delta_{X_{(r)}}^{(i)} / X_{(r-1)} \stackrel{\tau_{X_{(r-1)}\{i, j\}}}{\longrightarrow} \Pi_{X_{(r)}} / \Delta_{X_{(r)} / X_{(r-1)}}^{(j)} \\
& \text { via } \alpha_{(r)} \\
& \Pi_{Y_{(r)}} / \Delta_{Y_{(r)} / Y_{(r-1)}}^{(\sigma(i))} \underset{\tau_{Y_{(r-1)}\{\sigma(i), \sigma(j)\}}}{\longrightarrow} \Pi_{Y_{(r)}} / \Delta_{Y_{(r)} / Y_{(r-1)}}^{(\sigma(j))} .
\end{aligned}
$$

Here, the vertical arrows are the isomorphisms induced by $\alpha_{(r)}(c f$. (i)). 
Proof. Assertion (i) follows from the fact that an isomorphism of $\Pi_{X_{(r)}}$ with $\Pi_{Y_{(r)}}$ induces an isomorphism of $\Delta_{X_{(r)}}$ with $\Delta_{Y_{(r)}}$, together with [17], Corollary 6.7.

Next, we prove assertion (ii). If $r=1$, then this follows from [16], Remark 10, (iii). (Note that this essentially follows from [24], Corollary 2.10; Proposition 3.8.) Thus, assume that $r \geq 2$. Then it is immediate that for a section $s: G_{K} \rightarrow \Pi_{X_{(r)}}$ of the natural morphism $\Pi_{X_{(r)}} \rightarrow G_{K}$, the section arises from a $K$-rational point of $U_{X_{(r)}}$ if and only if the composite of the section $s$ and the morphism $\Pi_{X_{(r)}} \rightarrow \Pi_{X_{(r-1)}}$ induced by $p_{X_{(r-1)}: r}^{\log }$ arises from a $K$-rational point of $U_{X_{(r-1)}}$, and the section $G_{K} \rightarrow \Pi_{X_{(r)}} \times_{\Pi_{X_{(r-1)}}} G_{K}$ (where the morphism $\Pi_{X_{(r)}} \rightarrow \Pi_{X_{(r-1)}}$ is the morphism induced by $p_{X_{(r-1)}: r}^{\log }$, and $G_{K} \rightarrow \Pi_{X_{(r-1)}}$ is the composite) induced by the given section $s$ arises from a $K$-rational point of the hyperbolic curve obtained as the fiber. Therefore, assertion (ii) follows from [16], Remark 10, (iii), together with induction on $r$.

Next, we prove assertion (iii). It is immediate that there exists an open subgroup $G_{K^{\prime}} \subseteq G_{K}$ and a section $G_{K^{\prime}} \rightarrow \Pi_{X_{(r)}} \times_{G_{K}} G_{K^{\prime}}$ such that this section arises from a $K^{\prime}$-rational point of $U_{X_{(r)}}$. Thus, it follows from assertion (ii), the fact that $\mathfrak{I}_{X_{(r)}\{i, j\}}$ is an inertia subgroup of $\Pi_{X_{(r)}} \times_{\Pi_{X_{(r-1)}}} G_{K^{\prime}}$ (where the morphism $\Pi_{X_{(r)}} \rightarrow \Pi_{X_{(r-1)}}$ is the morphism induced by $p_{X_{(r-1)}: r}^{\log }$, and $G_{K^{\prime}} \rightarrow$ $\Pi_{X_{(r-1)}}$ is the composite of the section and the morphism induced by $\left.p_{X_{(r-1)}: r}^{\log }\right)$ associated to a cusp of the hyperbolic curve obtained as the fiber, together with a similar argument to the argument used in the proof of [13], Lemma 1.3.9, that $\alpha_{(r)}$ maps $\mathfrak{I}_{X_{(r)}\{i, j\}}$ bijectively onto a conjugate (in $\Delta_{Y_{(r)} / Y_{(r-1)}}^{(\sigma(i))}$ ) of $\mathfrak{I}_{Y_{(r)}\{\sigma(i), \sigma(j)\}}$. On the other hand, the assertion that $\alpha_{(r)}$ maps $\mathfrak{D}_{X_{(r)}\{i, j\}}$ bijectively onto a conjugate (in $\Delta_{Y_{(r)} / Y_{(r-1)}}^{(\sigma(i))}$ ) of $\mathfrak{D}_{Y_{(r)}\{\sigma(i), \sigma(j)\}}$ follows from the fact that $\alpha_{(r)}$ maps $\mathfrak{I}_{X_{(r)}\{i, j\}}$ bijectively onto a conjugate (in $\Delta_{Y_{(r)} / Y_{(r-1)}}^{(\sigma(i))}$ ) of $\mathfrak{I}_{Y_{(r)}\{\sigma(i), \sigma(j)\}}$, together with Lemma 2.2. This completes the proof of assertion (iii).

Finally, we prove assertion (iv). By the discussion preceding Definition 2.2, we have commutative diagrams

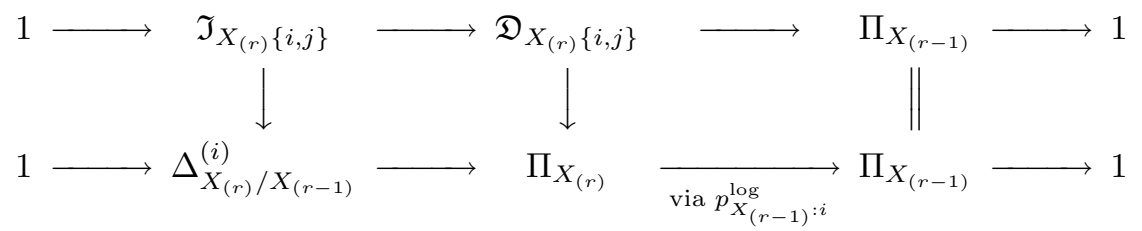


and

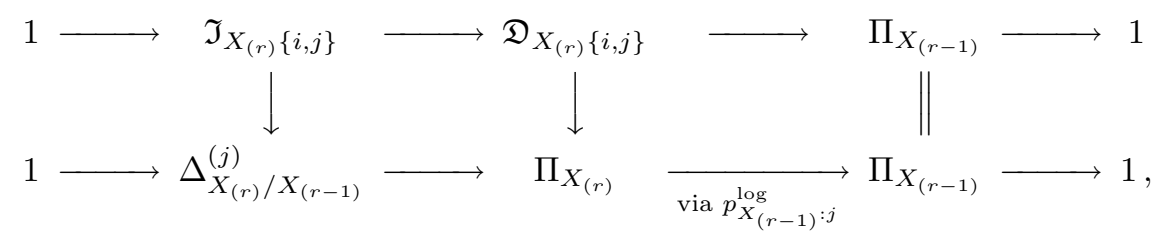

where the horizontal sequences are exact. In particular, the natural inclusion $\mathfrak{D}_{X_{(r)}\{i, j\}} \hookrightarrow \Pi_{X_{(r)}}$ induces isomorphisms

$$
\mathfrak{D}_{X_{(r)}\{i, j\}} / \mathfrak{I}_{X_{(r)}\{i, j\}} \stackrel{\sim}{\longrightarrow} \Pi_{X_{(r)}} / \Delta_{X_{(r)}}^{(i)} / X_{(r-1)}
$$

and

$$
\mathfrak{D}_{X_{(r)}\{i, j\}} / \mathfrak{I}_{X_{(r)}\{i, j\}} \stackrel{\sim}{\longrightarrow} \Pi_{X_{(r)}} / \Delta_{X_{(r)}}^{(j)} / X_{(r-1)} .
$$

Thus, the isomorphism $\tau_{X_{(r-1)}}\{i, j\}$ coincides with the composite

$$
\Pi_{X_{(r)}} / \Delta_{X_{(r)} / X_{(r-1)}}^{(i)} \stackrel{\sim}{\longleftarrow} \mathfrak{D}_{X_{(r)}\{i, j\}} / \mathfrak{I}_{X_{(r)}\{i, j\}} \stackrel{\sim}{\longrightarrow} \Pi_{X_{(r)}} / \Delta_{X_{(r)} / X_{(r-1)}}^{(i)} .
$$

Therefore, to verify the commutativity of the diagram in the statement of Proposition 2.5, (iv), it is enough to show that the isomorphism $\alpha_{(r)}$ maps $\mathfrak{D}_{X_{(r)}\{i, j\}}$ (respectively, $\mathfrak{I}_{X_{(r)}\{i, j\}}$ ) bijectively onto a conjugate of $\mathfrak{D}_{Y_{(r)}}\{\sigma(i), \sigma(j)\}$ (respectively, $\left.\mathfrak{I}_{Y_{(r)}\{\sigma(i), \sigma(j)\}}\right)$. On the other hand, this follows from (iii).

Definition 2.6. Let $\alpha_{(r)}: \Pi_{X_{(r)}} \stackrel{\sim}{\rightarrow} \Pi_{Y_{(r)}}$ be an isomorphism.

(i) We shall denote by $\sigma_{\alpha_{(r)}}$ the element of the symmetric group on $r$ letters defined in Proposition 2.5, (i).

(ii) We shall say that $\alpha_{(r)}$ is order-preserving if $\sigma_{\alpha_{(r)}}$ (defined in (i)) is the identity morphism. Note that by reordering the coordinates of $U_{Y_{(r)}}$, one can always assume that $\alpha_{(r)}$ is order-preserving.

Let $\alpha_{(r)}: \Pi_{X_{(r)}} \stackrel{\sim}{\rightarrow} \Pi_{Y_{(r)}}$ be a Frobenius-preserving and order-preserving isomorphism. Now by means of the isomorphism $\tau_{X_{(r-1)}\{i, j\}}$ (respectively, $\tau_{Y_{(r-1)}\{i, j\}}$ ) defined in Proposition 2.5, (iv), we identify the quotients $\Pi_{X_{(r)}} / \Delta_{X_{(r)} / X_{(r-1)}}^{(i)}$ (respectively, $\Pi_{Y_{(r)}} / \Delta_{Y_{(r)} / Y_{(r-1)}}^{(i)}$ ), where $i=1, \cdots, r$, of $\Pi_{X_{(r)}}$ (respectively, $\left.\Pi_{Y_{(r)}}\right)$; moreover, we also identify $\Pi_{X_{(r-1)}}$ (respectively, $\Pi_{Y_{(r-1)}}$ ) with these quotients $\Pi_{X_{(r)}} / \Delta_{X_{(r)} / X_{(r-1)}}^{(i)}$ (respectively, $\left.\Pi_{Y_{(r)}} / \Delta_{Y_{(r)} / Y_{(r-1)}}^{(i)}\right)$. We denote by $\alpha_{(r-1)}$ the isomorphism of $\Pi_{X_{(r-1)}}$ with $\Pi_{Y_{(r-1)}}$ induced by $\alpha_{(r)}$ (cf. Proposition 2.5, (i)). Note that this isomorphism 
$\alpha_{(r-1)}$ is independent of $i$. Moreover, by a similar argument to this argument, for any positive integer $r^{\prime} \leq r$, we obtain a quotient $\Pi_{X_{\left(r^{\prime}\right)}}$ (respectively, $\Pi_{Y_{\left(r^{\prime}\right)}}$ ) of $\Pi_{X_{(r)}}\left(\right.$ respectively, $\Pi_{Y_{(r)}}$ ) and an isomorphism $\alpha_{\left(r^{\prime}\right)}: \Pi_{X_{\left(r^{\prime}\right)}} \stackrel{\sim}{\rightarrow} \Pi_{Y_{\left(r^{\prime}\right)}}$. Note that it follows immediately from the definition of the term "Frobeniuspreserving" that the isomorphism $\alpha_{\left(r^{\prime}\right)}: \Pi_{X_{\left(r^{\prime}\right)}} \stackrel{\sim}{\rightarrow} \Pi_{Y_{\left(r^{\prime}\right)}}\left(\right.$ where $\left.r^{\prime} \leq r\right)$ is also Frobenius-preserving.

\section{§3. Isomorphisms That Preserve the Fundamental Groups of Tripods}

In this Section, we define the notion of a tripod-preserving isomorphism (where we refer to the discussion entitled "Curves" in Introduction concerning the term "tripod").

In the following, let $K$ (respectively, $L$ ) be a finite field whose cardinality we denote by $q_{K}$ (respectively, $q_{L}$ ), $\bar{K}$ (respectively, $\bar{L}$ ) an algebraic closure of $K$ (respectively, $L$ ), $X$ (respectively, $Y$ ) a proper hyperbolic curve of genus $g_{X} \geq 2$ (respectively, $g_{Y} \geq 2$ ) over $K$ (respectively, $L$ ), and $l$ a prime number which is invertible in $K$ and $L$. Let us write $G_{K} \stackrel{\text { def }}{=} \operatorname{Gal}(\bar{K} / K)$ and $G_{L} \stackrel{\text { def }}{=} \operatorname{Gal}(\bar{L} / L)$. Moreover, let us denote by $\Pi_{\mathbb{P}_{K}}$ (respectively, $\Pi_{\mathbb{P}_{L}}$ ) the geometrically pro-l $\log$ fundamental group of the $\log$ scheme $\mathbb{P}_{K}^{\log }$ (respectively, $\mathbb{P}_{L}^{\log }$ ) obtained by equipping $\mathbb{P}_{K}^{1}$ (respectively, $\mathbb{P}_{L}^{1}$ ) with the log structure associated to the divisor $\{0,1, \infty\}$, and by $\Delta_{\mathbb{P}_{K}}$ (respectively, $\Delta_{\mathbb{P}_{L}}$ ) the kernel of the natural surjection $\Pi_{\mathbb{P}_{K}} \rightarrow G_{K}$ (respectively, $\Pi_{\mathbb{P}_{L}} \rightarrow G_{L}$ ).

Write $E=\left\{e_{1}, e_{2}, e_{3}\right\} \stackrel{\text { def }}{=} \mathbb{P}_{K}^{1}(\bar{K}) \backslash U_{\mathbb{P}}(\bar{K})$ (where $U_{\mathbb{P}} \subseteq \mathbb{P}_{K}^{1}$ is the interior of $\mathbb{P}_{K}^{\log }$, i.e., $U_{\mathbb{P}}=\mathbb{P}_{K}^{1} \backslash\{0,1, \infty\}$ ), and $\mathfrak{I}_{e_{i}} \subseteq \Delta_{\mathbb{P}_{K}}$ (where $i=1,2,3$ ) for an inertia subgroup associated to $e_{i} \in E$ (well-defined, up to conjugation in $\Delta_{\mathbb{P}_{K}}$ ). Then it follows from the well-known structure of the pro-l fundamental group of the projective line minus three points over an algebraically closed field of characteristic $\neq l$ that the composites

$$
\mathfrak{I}_{e_{i}} \hookrightarrow \Delta_{\mathbb{P}_{K}} \rightarrow\left(\Delta_{\mathbb{P}_{K}}\right)^{\mathrm{ab}}
$$

induce an isomorphism

$$
\mathfrak{I}_{e_{1}} \oplus \mathfrak{I}_{e_{2}} \stackrel{\sim}{\longrightarrow}\left(\Delta_{\mathbb{P}_{K}}\right)^{\mathrm{ab}},
$$

where $e_{1} \neq e_{2}$. Moreover, there exists a generator $\zeta_{e_{i}} \in \mathfrak{I}_{e_{i}}(i=1,2,3)$ such that the image of $\zeta_{e_{3}}$ via the composite

$$
\mathfrak{I}_{e_{3}} \hookrightarrow \Delta_{\mathbb{P}_{K}} \rightarrow\left(\Delta_{\mathbb{P}_{K}}\right)^{\mathrm{ab}} \stackrel{\sim}{\sim} \mathfrak{I}_{e_{1}} \oplus \mathfrak{I}_{e_{2}}
$$


is $\left(-\zeta_{e_{1}},-\zeta_{e_{2}}\right) \in \mathfrak{I}_{e_{1}} \oplus \mathfrak{I}_{e_{2}}$, i.e., the image of the above composite is generated by $\left(\zeta_{e_{1}}, \zeta_{e_{2}}\right) \in \mathfrak{I}_{e_{1}} \oplus \mathfrak{I}_{e_{2}}$. Thus, if an automorphism $\bar{\phi}$ of $\left(\Delta_{\mathbb{P}_{K}}\right)^{\text {ab }}$ maps the image of $\mathfrak{I}_{e_{i}}$ in $\left(\Delta_{\mathbb{P}_{K}}\right)^{\text {ab }}(i=1,2,3)$ bijectively onto the image of $\mathfrak{I}_{\sigma\left(e_{i}\right)}$ in $\left(\Delta_{\mathbb{P}_{K}}\right)^{\mathrm{ab}}$ (where $\sigma$ is an element of the group $\operatorname{Aut}(E)$ of automorphisms of $E$ ), then there exists a unique element $d_{\bar{\phi}} \in \mathbb{Z}_{l}^{*}$ such that

$$
\bar{\phi}\left(\zeta_{e_{i}}\right)=d_{\bar{\phi}} \cdot \zeta_{\sigma\left(e_{i}\right)}(i=1,2,3) .
$$

Let $\phi: \prod_{\mathbb{P}_{K}} \stackrel{\sim}{\rightarrow} \prod_{\mathbb{P}_{K}}$ be a Frobenius-preserving automorphism (cf. Definition 2.5). Then the automorphism $\phi$ preserves the inertia subgroups up to conjugation. (Indeed, this follows from a similar argument to the argument used in the proof of [13], Lemma 1.3.9.) Therefore, by the above observation, we obtain an element $d_{\bar{\phi}} \in \mathbb{Z}_{l}^{*}$, where $\bar{\phi}$ is the automorphism of $\left(\Delta_{\mathbb{P}_{K}}\right)^{\text {ab }}$ induced by $\phi$.

Next, let $\phi: \Pi_{\mathbb{P}_{K}} \stackrel{\sim}{\rightarrow} \Pi_{\mathbb{P}_{L}}$ be a Frobenius-preserving isomorphism. Then it follows from the existence of such an isomorphism that $q_{K}=q_{L}$ (by considering the action of the respective Frobenius elements on $\left(\Delta_{\mathbb{P}_{K}}\right)^{\text {ab }}$ and $\left.\left(\Delta_{\mathbb{P}_{L}}\right)^{\mathrm{ab}}\right)$. In particular, the fields $K$ and $L$ are isomorphic. By means of some isomorphism of fields $K \stackrel{\sim}{\rightarrow} L$, we obtain an isomorphism $\Pi_{\mathbb{P}_{K}} \stackrel{\sim}{\rightarrow} \Pi_{\mathbb{P}_{L}}$.

In summary, we obtain a composite map

$$
\begin{aligned}
& \operatorname{Isom}_{\text {Frob }}\left(\Pi_{\mathbb{P}_{K}}, \Pi_{\mathbb{P}_{L}}\right) / \operatorname{Inn}\left(\Delta_{\mathbb{P}_{L}}\right) \stackrel{\sim}{\longrightarrow} \operatorname{Aut}_{\text {Frob }}\left(\Pi_{\mathbb{P}_{K}}\right) / \operatorname{Inn}\left(\Delta_{\mathbb{P}_{K}}\right) \\
& \longrightarrow \operatorname{Aut}_{\text {Iner }}\left(\left(\Delta_{\mathbb{P}_{K}}\right)^{\mathrm{ab}}\right) \longrightarrow \mathbb{Z}_{l}^{*} \\
& \mapsto d_{\bar{\phi}},
\end{aligned}
$$

where $\operatorname{Isom}_{\text {Frob }}\left(\Pi_{\mathbb{P}_{K}}, \Pi_{\mathbb{P}_{L}}\right)$ (respectively, $\left.\operatorname{Aut}_{\text {Frob }}\left(\Pi_{\mathbb{P}_{K}}\right)\right)$ is the set of Frobeniuspreserving isomorphisms (respectively, automorphisms) of $\Pi_{\mathbb{P}_{K}}$ with $\Pi_{\mathbb{P}_{L}}$ (respectively, of $\left.\Pi_{\mathbb{P}_{K}}\right)$, Aut $\left.\operatorname{Iner}_{(}\left(\Delta_{\mathbb{P}_{K}}\right)^{\mathrm{ab}}\right)$ is the set of automorphisms of $\left(\Delta_{\mathbb{P}_{K}}\right)^{\mathrm{ab}}$ which preserve the images of the three inertia subgroups in $\left(\Delta_{\mathbb{P}_{K}}\right)^{\text {ab }}$, and the first arrow is the bijection induced by some isomorphism of fields $K \stackrel{\sim}{\rightarrow} L$. Note that this composite depends on the choice of an isomorphism of $K$ with $L$; however, the image of this composite is independent of the choice of an isomorphism of $K$ with $L$.

Definition 3.1. We shall refer to the image $\operatorname{Im}\left(\operatorname{deg}_{\mathbb{P}}\right) \subseteq \mathbb{Z}_{l}^{*}$ of this composite

$$
\begin{aligned}
& \operatorname{deg}_{\mathbb{P}}: \operatorname{Isom}_{\text {Frob }}\left(\Pi_{\mathbb{P}_{K}}, \Pi_{\mathbb{P}_{L}}\right) / \operatorname{Inn}\left(\Delta_{\mathbb{P}_{L}}\right) \stackrel{\sim}{\longrightarrow} \operatorname{Aut}_{\text {Frob }}\left(\Pi_{\mathbb{P}_{K}}\right) / \operatorname{Inn}\left(\Delta_{\mathbb{P}_{K}}\right) \\
& \longrightarrow \operatorname{Aut}_{\operatorname{Iner}}\left(\left(\Delta_{\mathbb{P}_{K}}\right)^{\mathrm{ab}}\right) \longrightarrow \mathbb{Z}_{l}^{*} \\
& \bar{\phi} \mapsto d_{\bar{\phi}}
\end{aligned}
$$


as the set of tripod-degrees (over $K$ ). We shall refer to an element of the set of tripod-degrees (over $K$ ) as a tripod-degree (over $K$ ).

\section{Remark 6.}

(i) The set of tripod-degrees (over $K)$ only depends on $K(\simeq L)$ and $l$.

(ii) Since the image of the composite

$$
\operatorname{Isom}\left(\mathbb{P}_{K}^{\log }, \mathbb{P}_{L}^{\log }\right) \longrightarrow \operatorname{Isom}_{\mathrm{Frob}}\left(\Pi_{\mathbb{P}_{K}}, \Pi_{\mathbb{P}_{L}}\right) / \operatorname{Inn}\left(\Delta_{\mathbb{P}_{L}}\right) \stackrel{\operatorname{deg}_{\mathbb{P}}}{\longrightarrow} \mathbb{Z}_{l}^{*}
$$

(where $\operatorname{Isom}\left(\mathbb{P}_{K}^{\log }, \mathbb{P}_{L}^{\log }\right)$ is the set of isomorphisms of $\mathbb{P}_{K}^{\log }$ with $\mathbb{P}_{L}^{\log }$ [as log schemes], the first arrow is the morphism induced by the functoriality of the functor of taking the log fundamental group) is the subgroup $\left\langle q_{K}\right\rangle$ generated by $q_{K} \in \mathbb{Z}_{l}^{*}$, the set of tripod-degrees (over $K$ ) includes $\left\langle q_{K}\right\rangle \subseteq$ $\mathbb{Z}_{l}^{*}$.

(iii) By an unpublished result of Akio Tamagawa, in general, the set of tripoddegrees (over $K$ ) is a proper subset of $\mathbb{Z}_{l}^{*}$.

(iv) The morphism $\operatorname{deg}_{\mathbb{P}}: \operatorname{Isom}_{\mathrm{Frob}}\left(\Pi_{\mathbb{P}_{K}}, \Pi_{\mathbb{P}_{L}}\right) / \operatorname{Inn}\left(\Delta_{\mathbb{P}_{L}}\right) \rightarrow \mathbb{Z}_{l}^{*}$ is "essentially" injective, i.e., if we denote by

$$
\operatorname{Isom}_{\mathrm{Frob}}^{\{0,1, \infty\}}\left(\Pi_{\mathbb{P}_{K}}, \Pi_{\mathbb{P}_{L}}\right)
$$

the subset of $\operatorname{Isom}_{\mathrm{Frob}}\left(\Pi_{\mathbb{P}_{K}}, \Pi_{\mathbb{P}_{L}}\right)$ consisting of isomorphisms which map the decomposition subgroup $\mathfrak{D}_{e} \subseteq \Pi_{\mathbb{P}_{K}}$ associated to $e \in\{0,1, \infty\}$ (welldefined, up to conjugation) to a $\Delta_{\mathbb{P}_{L}}$-conjugate of the decomposition subgroup $\mathfrak{D}_{e} \subseteq \Pi_{\mathbb{P}_{L}}$ associated to $e$ (well-defined, up to conjugation), then the composite

$$
\operatorname{Isom}_{\text {Frob }}^{\{0,1, \infty\}}\left(\Pi_{\mathbb{P}_{K}}, \Pi_{\mathbb{P}_{L}}\right) / \operatorname{Inn}\left(\Delta_{\mathbb{P}_{L}}\right) \hookrightarrow \operatorname{Isom}_{\mathrm{Frob}}\left(\Pi_{\mathbb{P}_{K}}, \Pi_{\mathbb{P}_{L}}\right) / \operatorname{Inn}\left(\Delta_{\mathbb{P}_{L}}\right) \stackrel{\operatorname{deg}_{\mathbb{P}}}{\longrightarrow} \mathbb{Z}_{l}^{*}
$$

is injective. Indeed, by a similar argument to the argument used in the proof of the uniqueness of " $\alpha_{\infty}$ " in [16], Theorem 3.1, to prove this injectivity, it is enough to show the following assertion:

For any Frobenius-preserving automorphism $\alpha$ of $\Pi_{\mathbb{P}_{K}}$ which preserves a fixed decomposition subgroup $\mathfrak{D}_{0} \subseteq \Pi_{\mathbb{P}_{K}}$ associated to $0 \in \mathbb{P}_{K}^{1}(K)$ and induces the identity morphism on $\mathfrak{I}_{0}$, there exists a section of the natural surjection $\mathfrak{D}_{0} \rightarrow G_{K}$ which is preserved by $\alpha$. 
This assertion is verified as follows (cf. the theory of [14], Section 4): Let $\operatorname{Sec}\left(\mathfrak{D}_{0} \rightarrow G_{K}\right)$ (respectively, $\operatorname{Sec}\left(\mathfrak{D}_{0} \times_{G_{K}} \mathfrak{D}_{0} \stackrel{\mathrm{pr}_{2}}{\rightarrow} \mathfrak{D}_{0}\right)$ ) be the set of sections of the natural surjection $\mathfrak{D}_{0} \rightarrow G_{K}$ (respectively, $\mathfrak{D}_{0} \times_{G_{K}} \mathfrak{D}_{0} \stackrel{\mathrm{pr}_{2}}{\rightarrow}$ $\left.\mathfrak{D}_{0}\right)$. Then by considering the difference between an arbitrary element of $\operatorname{Sec}\left(\mathfrak{D}_{0} \times G_{K} \mathfrak{D}_{0} \stackrel{\operatorname{pr}_{2}}{\rightarrow} \mathfrak{D}_{0}\right)$ and the element determined by the diagonal morphism, we obtain a bijection of $\operatorname{Sec}\left(\mathfrak{D}_{0} \times G_{K} \mathfrak{D}_{0} \stackrel{\operatorname{pr}_{2}}{\rightarrow} \mathfrak{D}_{0}\right)$ with $H^{1}\left(\mathfrak{D}_{0}, \mathfrak{I}_{0}\right)$. Moreover, it is easily verified that under this bijection, the $\operatorname{subset} \operatorname{Sec}\left(\mathfrak{D}_{0} \rightarrow\right.$ $\left.G_{K}\right) \subseteq \operatorname{Sec}\left(\mathfrak{D}_{0} \times_{G_{K}} \mathfrak{D}_{0} \stackrel{\mathrm{pr}_{2}}{\rightarrow} \mathfrak{D}_{0}\right)$ (where the inclusion is obtained by taking the pull-back) corresponds to $H_{\mathfrak{I}_{0}} \stackrel{\text { def }}{=}\left\{\lambda \in H^{1}\left(\mathfrak{D}_{0}, \mathfrak{I}_{0}\right)|\lambda|_{\mathfrak{I}_{0}}=\mathrm{id}_{\mathfrak{I}_{0}}\right\}$ (where $\left.\lambda\right|_{\mathfrak{I}_{0}}$ is the image of $\lambda$ in $H^{1}\left(\mathfrak{I}_{0}, \mathfrak{I}_{0}\right) \simeq \operatorname{Hom}\left(\mathfrak{I}_{0}, \mathfrak{I}_{0}\right)$ ), i.e., we obtain a natural bijection

$$
\operatorname{Sec}\left(\mathfrak{D}_{0} \rightarrow G_{K}\right) \simeq H_{\mathfrak{I}_{0}} .
$$

Thus, to prove the assertion, it is enough to show that there exists an element of $H_{\mathfrak{I}_{0}}$ which is preserved by the automorphism of $H_{\mathfrak{I}_{0}}$ induced by $\alpha$. Now we fix an isomorphism $\mathfrak{I}_{0} \stackrel{\sim}{\rightarrow} \mathbb{Z}_{l}(1)$. Then if $u$ is the canonical coordinate of $\mathbb{P}_{K}^{1}$ (i.e., $\left.U_{\mathbb{P}}=\operatorname{Spec} K\left[u, u^{-1},(u-1)^{-1}\right]\right)$, and $\kappa(u) \in H^{1}\left(\Pi_{\mathbb{P}_{K}}, \mathfrak{I}_{0}\right)$ is the Kummer class of $u$ (i.e., the image of $u \in H^{0}\left(U_{\mathbb{P}}, \mathbb{G}_{m}\right)$ in $H^{1}\left(\Pi_{\mathbb{P}_{K}}, \mathfrak{I}_{0}\right)$ via the morphism obtained by considering the Kummer exact sequence, together with the fixed isomorphism $\mathfrak{I}_{0} \stackrel{\sim}{\rightarrow} \mathbb{Z}_{l}(1)$ ), then it is easily verified that $\kappa(u)$ satisfies the following condition:

The image of $\kappa(u)$ in $H^{1}\left(\mathfrak{I}_{0}, \mathfrak{I}_{0}\right) \simeq \operatorname{Hom}\left(\mathfrak{I}_{0}, \mathfrak{I}_{0}\right)$ (respectively, $\left.H^{1}\left(\mathfrak{I}_{1}, \mathfrak{I}_{0}\right) \simeq \operatorname{Hom}\left(\mathfrak{I}_{1}, \mathfrak{I}_{0}\right)\right)$ is the identity morphism of $\mathfrak{I}_{0}$, i.e., $\kappa(u) \in H_{\mathfrak{I}_{0}}$ (respectively, zero, and the element in $\left(K^{*}\right)^{(l)}[\simeq$ $\left.H^{1}\left(K, \mathbb{Z}_{l}(1)\right) \stackrel{\sim}{\leftarrow} H^{1}\left(K, \mathfrak{I}_{0}\right)\right]$ obtained by considering the exact sequence

$$
0 \longrightarrow\left(K^{*}\right)^{(l)} \longrightarrow H^{1}\left(\mathfrak{D}_{1}, \mathfrak{I}_{0}\right) \longrightarrow H^{1}\left(\mathfrak{I}_{1}, \mathfrak{I}_{0}\right)
$$

is $\left.1 \in\left(K^{*}\right)^{(l)}\right)$.

Moreover, (since it is easily verified that the natural morphism $H^{1}\left(\Pi_{\mathbb{P}_{K}}, \mathfrak{I}_{0}\right)$ $\rightarrow H^{1}\left(\mathfrak{I}_{0}, \mathfrak{I}_{0}\right) \oplus H^{1}\left(\mathfrak{D}_{1}, \mathfrak{I}_{0}\right)$ is an isomorphism $) \kappa(u) \in H^{1}\left(\Pi_{\mathbb{P}_{K}}, \mathfrak{I}_{0}\right)$ is uniquely determined by this condition; in particular, the automorphism of $H^{1}\left(\Pi_{\mathbb{P}_{K}}, \Im_{0}\right)$ induced by $\alpha$ preserves $\kappa(u)$. Therefore, the assertion follows from the fact that the section of $\mathfrak{D}_{0} \rightarrow G_{K}$ determined by the image of $\kappa(u)$ in $H^{1}\left(\mathfrak{D}_{0}, \mathfrak{I}_{0}\right)$ is preserved by $\alpha$.

Note that another proof of the fact that the composite $\operatorname{Isom}_{\text {Frob }}^{\{0,1, \infty\}}\left(\Pi_{\mathbb{P}_{K}}, \Pi_{\mathbb{P}_{L}}\right) / \operatorname{Inn}\left(\Delta_{\mathbb{P}_{L}}\right) \hookrightarrow \operatorname{Isom}_{\text {Frob }}\left(\Pi_{\mathbb{P}_{K}}, \Pi_{\mathbb{P}_{L}}\right) / \operatorname{Inn}\left(\Delta_{\mathbb{P}_{L}}\right) \longrightarrow \mathbb{Z}_{l}^{*}$ 
is injective can be found in the latter half of the proof of [20], Lemma (2.2.4).

Next, let $\alpha: \Pi_{X} \stackrel{\sim}{\rightarrow} \Pi_{Y}$ be a Frobenius-preserving isomorphism. Then it follows from the existence of the isomorphism $\alpha$ that $q_{K}=q_{L}$ (by considering the actions of the respective Frobenius elements on $H^{2}\left(\Delta_{X}, \mathbb{Z}_{l}\right)$ and $\left.H^{2}\left(\Delta_{Y}, \mathbb{Z}_{l}\right)\right)$. In particular, the fields $K$ and $L$ are isomorphic. By means of some isomorphism of fields $K \stackrel{\sim}{\rightarrow} L$, we obtain an isomorphism $\beta: \mathbb{P}_{K}^{\log } \stackrel{\sim}{\rightarrow} \mathbb{P}_{L}^{\log }$. Now by considering the composite of the morphism $m_{X_{(r+1)}\{1,2\}}: M_{X} \stackrel{\text { def }}{=}$ $\left.\operatorname{Hom}_{\mathbb{Z}_{l}}\left(H^{2}\left(\Delta_{X}, \mathbb{Z}_{l}\right), \mathbb{Z}_{l}\right)\right) \stackrel{\sim}{\rightarrow} \mathfrak{I}_{X_{(r+1)}\{1,2\}} \quad\left(\right.$ respectively, $m_{Y_{(r+1)}\{1,2\}}: M_{Y} \stackrel{\text { def }}{=}$ $\left.\left.\operatorname{Hom}_{\mathbb{Z}_{l}}\left(H^{2}\left(\Delta_{Y}, \mathbb{Z}_{l}\right), \mathbb{Z}_{l}\right)\right) \stackrel{\sim}{\rightarrow} \mathfrak{I}_{Y_{(r+1)}\{1,2\}}\right)$ (cf. Definition 4.2, (i), (ii) below) and the isomorphism of $\mathfrak{I}_{X_{(r+1)}\{1,2\}}$ (respectively, $\mathfrak{I}_{Y_{(r+1)}\{1,2\}}$ ) with an inertia subgroup of $\Delta_{\mathbb{P}_{K}}$ (respectively, $\Delta_{\mathbb{P}_{L}}$ ) obtained in Lemma 2.1, we obtain a natural isomorphism of $M_{X}$ (respectively, $M_{Y}$ ) with an inertia subgroup of $\Delta_{\mathbb{P}_{K}}$ (respectively, $\Delta_{\mathbb{P}_{L}}$ ). Thus, by means of the isomorphism $\beta: \mathbb{P}_{K}^{\log } \stackrel{\sim}{\rightarrow} \mathbb{P}_{L}^{\log }$, we obtain an isomorphism $M_{X} \stackrel{\sim}{\rightarrow} M_{Y}$ (cf. Remark 7 below). Therefore, we obtain a composite map

$$
\operatorname{Isom}_{\text {Frob }}\left(\Pi_{X}, \Pi_{Y}\right) / \operatorname{Inn}\left(\Delta_{Y}\right) \longrightarrow \operatorname{Isom}\left(M_{X}, M_{Y}\right) \stackrel{\sim}{\longrightarrow} \operatorname{Aut}\left(M_{X}\right) \stackrel{\sim}{\longrightarrow} \mathbb{Z}_{l}^{*},
$$

where $\operatorname{Isom}_{\operatorname{Frob}}\left(\Pi_{X}, \Pi_{Y}\right)$ is the set of Frobenius-preserving isomorphisms of $\Pi_{X}$ with $\Pi_{Y}$, the second arrow is the bijection induced by some isomorphism of fields $K \stackrel{\sim}{\rightarrow} L$. Note that this composite depends on the choice of an isomorphism of $K$ with $L$; however, the image of this composite is independent of the choice of an isomorphism of $K$ with $L$.

\section{Remark 7.}

(i) Note that the isomorphism $M_{X} \stackrel{\sim}{\rightarrow} M_{Y}$ (obtained as above) is independent of $\alpha$ (by construction); moreover, this isomorphism is "geometric", i.e., it arises from an isomorphism $\mathbb{P}_{K}^{\log \stackrel{\sim}{\rightarrow}} \mathbb{P}_{L}^{\log }$.

(ii) The morphism

$$
\operatorname{Isom}\left(M_{X}, M_{Y}\right) \stackrel{\sim}{\longrightarrow} \operatorname{Aut}\left(M_{X}\right)
$$

(appearing in the above composite map) may be interpreted as a certain "automorphization" of isomorphisms of $M_{X}$ with $M_{Y}$ by means of the "geometric" isomorphism of (i), that is independent of $\alpha$.

Definition 3.2. $\quad$ Let $\alpha: \Pi_{X} \stackrel{\sim}{\rightarrow} \Pi_{Y}$ be a Frobenius-preserving isomorphism. 
(i) We shall denote by $\operatorname{deg}(\alpha) \in \mathbb{Z}_{l}^{*}$ the image of $\alpha$ via the composite

$$
\operatorname{Isom}_{\mathrm{Frob}}\left(\Pi_{X}, \Pi_{Y}\right) / \operatorname{Inn}\left(\Delta_{Y}\right) \longrightarrow \operatorname{Isom}\left(M_{X}, M_{Y}\right) \stackrel{\sim}{\longrightarrow} \operatorname{Aut}\left(M_{X}\right) \stackrel{\sim}{\longrightarrow} \mathbb{Z}_{l}^{*} .
$$

Note that $\operatorname{deg}(\alpha)$ depends on the choice of an isomorphism of $K$ with $L$.

(ii) We shall say that $\alpha: \Pi_{X} \stackrel{\sim}{\rightarrow} \Pi_{Y}$ is tripod-preserving if $\operatorname{deg}(\alpha)$ is a tripoddegree over $K$ (cf. Definition 3.1). Note that this condition is independent of the choice of an isomorphism of $K$ with $L$.

Next, let $\alpha_{(r)}: \Pi_{X_{(r)}} \stackrel{\sim}{\rightarrow} \Pi_{Y_{(r)}}$ be a Frobenius-preserving isomorphism.

Definition 3.3. We shall say that $\alpha_{(r)}$ is tripod-preserving if the isomorphism $\alpha: \Pi_{X} \stackrel{\sim}{\rightarrow} \Pi_{Y}$ induced by $\alpha_{(r)}$ (cf. the discussion following Definition 2.6) is tripod-preserving (cf. Definition 3.2, (ii)). Note that in fact, any Frobenius-preserving isomorphisms are tripod-preserving (cf. Lemma 4.17).

Lemma 3.1. If $r \geq 3$, then $\alpha_{(r)}$ is tripod-preserving.

Proof. To prove Lemma 3.1, by replacing $K$ by a finite extension field of $K$, we may assume without loss of generality that $U_{X_{(r-2)}}(K) \neq \emptyset$. Let $G_{K} \rightarrow \Pi_{X_{(r-2)}}$ be a section which arises from a $K$-rational point of $U_{X_{(r-2)}}$. By base-chaging this section via the composite

$$
\mathfrak{D}_{X_{(r-1)}\{1,2\}} \hookrightarrow \Pi_{X_{(r-1)}} \stackrel{\text { via }}{p_{X_{(r-2)}: 1}^{\log }} \Pi_{X_{(r-2)}},
$$

we obtain a morphism

$$
s: G_{K} \times_{\Pi_{X_{(r-2)}}} \mathfrak{D}_{X_{(r-1)}\{1,2\}} \stackrel{\mathrm{pr}_{2}}{\longrightarrow} \mathfrak{D}_{X_{(r-1)}\{1,2\}} \hookrightarrow \Pi_{X_{(r-1)}} .
$$

It is immediate that this morphism arises from a "strict log $K$-rational point" of $X_{(r-1)}^{\log }$ (i.e., a $K$-rational point of $X_{(r-1)}$ equipped with the log structure induced by the $\log$ structure of $\left.X_{(r-1)}^{\log }\right)$ for which the image of the underlying morphism of schemes lies in the open subscheme of $D_{X_{(r-1)}\{1,2\}}$ on which the stalk of the characteristic sheaf (where we refer to the discussion entitled "Log schemes" in Introduction concerning the term "characteristic sheaf") of $D_{X_{(r-1)}\{1,2\}}^{\log }$ is isomorphic to $\mathbb{N}$. Thus, the fiber product

$$
\left(G_{K} \times_{\Pi_{X_{(r-2)}}} \mathfrak{D}_{X_{(r-1)}\{1,2\}}\right) \times_{\Pi_{X_{(r-1)}}} \Pi_{X_{(r)}}
$$

(where the morphism $G_{K} \times_{\Pi_{X_{(r-2)}}} \mathfrak{D}_{X_{(r-1)}\{1,2\}} \rightarrow \Pi_{X_{(r-1)}}$ is $s$, and $\Pi_{X_{(r)}} \rightarrow$ $\Pi_{X_{(r-1)}}$ is the morphism induced by $\left.p_{X_{(r-1)}: 1}^{\log }\right)$ is isomorphic to the geometrically 
pro-l $l \log$ fundamental group of the $\log$ scheme obtained as the fiber of $p_{X_{(r-1)}: 1}^{\log }$ at the "strict $\log K$-rational point" of $X_{(r-1)}^{\log }$ corresponding to $s$, and the morphism

$$
\left(G_{K} \times_{\Pi_{X_{(r-2)}}} \mathfrak{D}_{X_{(r-1)}\{1,2\}}\right) \times_{\Pi_{X_{(r-1)}}} \Pi_{X_{(r)}} \stackrel{\mathrm{pr}_{1}}{\longrightarrow} G_{K} \times_{\Pi_{X_{(r-2)}}} \mathfrak{D}_{X_{(r-1)}\{1,2\}}
$$

coincides with the morphism induced by the structure morphism of the log scheme (obtained as the fiber of $p_{X_{(r-1): 1}}^{\log }$ at the "strict $\log K$-rational point" of $\left.X_{(r-1)}^{\log }\right)$. Now it is immediate that the underlying scheme of the log scheme obtained as such a fiber has exactly two irreducible components of genera 0 and $g_{X}$; moreover, if we denote by $H$ the closed subgroup of

$$
\left(G_{K} \times_{\Pi_{X_{(r-2)}}} \mathfrak{D}_{X_{(r-1)}\{1,2\}}\right) \times_{\Pi_{X_{(r-1)}}} \Pi_{X_{(r)}}
$$

(well-defined, up to conjugation) obtained as the image of the morphism induced on geometrically pro-l log fundamental groups by the strict closed immersion from the irreducible component of genus 0 , then the kernel $H^{\Delta}$ of the composite

$$
H \hookrightarrow\left(G_{K} \times_{\Pi_{X_{(r-2)}}} \mathfrak{D}_{X_{(r-1)}\{1,2\}}\right) \times_{\Pi_{X_{(r-1)}}} \Pi_{X_{(r)}} \stackrel{\mathrm{pr}_{1}}{\longrightarrow} G_{K} \times_{\Pi_{X_{(r-2)}}} \mathfrak{D}_{X_{(r-1)}\{1,2\}}
$$

is naturally isomorphic to $\Delta_{\mathbb{P}_{K}}$. On the other hand, it follows that the outer representation

$$
G_{K} \times_{\Pi_{X_{(r-2)}}} \mathfrak{D}_{X_{(r-1)}\{1,2\}} \stackrel{\rho_{H}}{\longrightarrow} \operatorname{Out}\left(H^{\Delta}\right)
$$

determined by the exact sequence

$$
1 \longrightarrow H^{\Delta} \longrightarrow H \longrightarrow G_{K} \times_{\Pi_{X_{(r-2)}}} \mathfrak{D}_{X_{(r-1)}\{1,2\}} \longrightarrow 1
$$

factors through $G_{K} \times_{\Pi_{X}(r-2)} \mathfrak{D}_{X_{(r-1)}\{1,2\}} \stackrel{\mathrm{pr}_{1}}{\rightarrow} G_{K}$, and the profinte group

$$
H^{\Delta} \stackrel{\text { out }}{\rtimes} G_{K}
$$

(where $G_{K} \rightarrow \operatorname{Out}\left(H^{\Delta}\right)$ is the morphism induced by $\rho_{H}$ ) is isomorphic to the geometrically pro-l fundamental group $\Pi_{\mathbb{P}_{K}}$ of $\mathbb{P}_{K}^{1} \backslash\{0,1, \infty\}$. Therefore, Lemma 3.1 follows from Proposition 2.5, (ii), (iii); [15], Corollary 2.8. 


\section{§4. The Reconstruction of the Fundamental Group of the Configuration Space}

In this Section, we reconstruct the geometrically pro-l fundamental group of the higher dimensional configuration space.

Let $K$ be a finite field whose characteristic (respectively, cardinality) we denote by $p_{K}$ (respectively, $q_{K}$ ), and $l$ a prime number that is invertible in $K$. We shall fix an algebraic closure $\bar{K}$ of $K$. We shall denote by $G_{K}$ the Galois group of $\bar{K}$ over $K$, and by $\operatorname{Fr}_{K} \in G_{K}$ the Frobenius element of $G_{K}$. Moreover, in the following, let $X$ be a proper hyperbolic curve of genus $g_{X} \geq 2$ over $K$.

Definition 4.1. Let $r$ be a natural number.

(i) We shall denote by

$$
\left\{\Delta_{X_{(r)}}(n)\right\}
$$

the central filtration of $\Delta_{X_{(r)}}$ defined in Definition 1.4, (ii), associated to the successive extension of hyperbolic curves of product type obtained as the base-change of

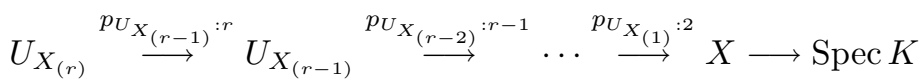

from $K$ to $\bar{K}$ i.e., the central filtration with respect to the natural surjection

$$
\Delta_{X_{(r)}} \rightarrow \Delta_{X_{(r)}}^{\mathrm{ab}},
$$

and by

$$
\left\{\Delta_{X_{(r)} / X_{(r-1)}}^{(i)}(n)\right\}
$$

the central filtration of $\Delta_{X_{(r)} / X_{(r-1)}}^{(i)}$ defined in the discussion following Lemma 1.1 associated to the family of curves

$$
U_{X_{(r)}} \otimes_{K} \bar{K} \stackrel{\text { via }}{p_{U_{(r-1)}}: i} U_{X_{(r-1)}} \otimes_{K} \bar{K}
$$

i.e., the central filtration with respect to the natural surjection

$$
\Delta_{X_{(r)} / X_{(r-1)}}^{(i)} \rightarrow \Delta_{X}^{\mathrm{ab}} .
$$

(ii) The sequence obtained as the base-change of

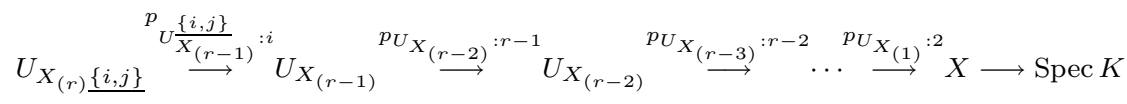


(cf. Definition 2.4, (i)) from $K$ to $\bar{K}$ is a successive extension of hyperbolic curves of product type. We shall denote by

$$
\left\{\Delta_{X_{(r)} \underline{\{i, j\}}}(n)\right\}
$$

the central filtration of $\Delta_{X_{(r)}\{i, j\}}$ defined in Definition 1.4, (ii), associated to this successive extension of hyperbolic curves of product type, i.e., the central filtration with respect to the natural surjection

$$
\Delta_{X_{(r) \underline{\{i, j\}}}} \rightarrow \Delta_{X_{\underline{(r)}}^{\mathrm{ab}}} .
$$

Proposition 4.1 (cf. [16], Proposition 3.1, (i)). Let $r$ be a natural number.

(i) The sequence of graded Lie algebras

$$
1 \longrightarrow \operatorname{Gr}\left(\Delta_{X_{(r)} / X_{(r-1)}}^{(i)}\right) \longrightarrow \operatorname{Gr}\left(\Delta_{X_{(r+1)}}\right) \stackrel{\text { via } p_{U_{X}(r)}{ }^{: i}}{\longrightarrow} \operatorname{Gr}\left(\Delta_{X_{(r)}}\right) \longrightarrow 1
$$

induced by the exact sequence obtained in Definition 2.1, (iv), is exact. In particular, the graded Lie algebra $\operatorname{Gr}\left(\Delta_{X_{(r)}}\right)$ is center-free.

(ii) There exist $2 g_{X}$ elements

$$
\alpha_{X, 1}^{(i)} ; \cdots ; \alpha_{X, g_{X}}^{(i)} ; \beta_{X, 1}^{(i)} ; \cdots ; \beta_{X, g_{X}}^{(i)} \in \Delta_{X_{(r+1)} / X_{(r)}}^{(i)} \backslash \Delta_{X_{(r+1)} / X_{(r)}}^{(i)}
$$

and $r$ elements

$$
\zeta_{X, 1}^{(i)} ; \cdots ; \zeta_{X, i-1}^{(i)} ; \zeta_{X, i+1}^{(i)} ; \cdots ; \zeta_{X, r+1}^{(i)} \in \Delta_{X_{(r+1)} / X_{(r)}}^{(i)}(2) \backslash \Delta_{X_{(r+1)} / X_{(r)}}^{(i)}(3)
$$

such that the graded Lie algebra $\operatorname{Gr}\left(\Delta_{X_{(r+1)} / X_{(r)}}^{(i)}\right)$ is generated by the images of these elements, and, moreover, $\zeta_{X, k}^{(i)}($ where $i \neq k)$ topologically generates the inertia subgroup of $\Delta_{X_{(r+1)}}^{(i)} / X_{(r)}$ (well-defined, up to conjugation) associated to the cusp (of the geometric fiber of $p_{U_{X_{(r)}}: i}: U_{X_{(r+1)}} \rightarrow U_{X_{(r)}}$ at a geometric point of $\left.U_{X_{(r)}}\right)$ determined by the divisor $D_{X_{(r+1)}}\{i, k\} \subseteq X_{(r+1)}$. Moreover, the graded Lie algebra $\operatorname{Gr}\left(\Delta_{X_{(r+1)}^{(i)} / X_{(r)}}^{(i)}\right)$ is isomorphic to the graded Lie algebra generated by these elements subject to the following relation, where "(-)" is the image of "(-)":

$$
\sum_{j=1}^{g_{X}}\left[\bar{\alpha}_{X, j}^{(i)}, \bar{\beta}_{X, j}^{(i)}\right]+\sum_{k \neq i} \bar{\zeta}_{X, k}^{(i)}=0 .
$$


(iii) The graded Lie algebra $\operatorname{Gr}\left(\Delta_{X_{(r+1)}}\right)$ is isomorphic to the graded Lie algebra generated by the images of

$\alpha_{X, 1}^{(i)} ; \cdots ; \alpha_{X, g_{X}}^{(i)} ; \beta_{X, 1}^{(i)} ; \cdots ; \beta_{X, g_{X}}^{(i)} \in \Delta_{X_{(r+1)} / X_{(r)}}^{(i)} \subseteq \Delta_{X_{(r+1)}}(1 \leq i \leq r+1)$,

together with

$\zeta_{X, 1}^{(i)} ; \cdots ; \zeta_{X, i-1}^{(i)} ; \zeta_{X, i+1}^{(i)} ; \cdots ; \zeta_{X, r+1}^{(i)} \in \Delta_{X_{(r+1)} / X_{(r)}}^{(i)} \subseteq \Delta_{X_{(r+1)}} \quad(1 \leq i \leq r+1)$

in (ii) subject to the following relations, where " $\overline{(-)}$ " is the image of " $(-)$ ":

$\left(R_{1}\right) \sum_{j=1}^{g_{X}}\left[\bar{\alpha}_{X, j}^{(i)}, \bar{\beta}_{X, j}^{(i)}\right]+\sum_{k \neq i} \bar{\zeta}_{X, k}^{(i)}=0(1 \leq i \leq r+1) ;$

$\left(R_{2}\right) \bar{\zeta}_{X, k}^{(i)}=\bar{\zeta}_{X, i}^{(k)}$

$\left(R_{3}\right)\left[\bar{\zeta}_{X, k}^{(i)}, \bar{\zeta}_{X, k^{\prime}}^{\left(i^{\prime}\right)}\right]=0$ (if $\left.\{i, k\} \cap\left\{i^{\prime}, k^{\prime}\right\}=\emptyset\right)$;

$\left(R_{4}\right)\left[\bar{\zeta}_{X, k}^{(i)}, \bar{\alpha}_{X, j}^{\left(i^{\prime}\right)}\right]=\left[\bar{\zeta}_{X, k}^{(i)}, \bar{\beta}_{X, j}^{\left(i^{\prime}\right)}\right]=0$ (if $i \neq i^{\prime}$ and $\left.k \neq i^{\prime}\right)$

$\left(R_{5}\right)\left[\bar{\alpha}_{X, j}^{(i)}, \bar{\alpha}_{X, j^{\prime}}^{\left(i^{\prime}\right)}\right]=\left[\bar{\beta}_{X, j}^{(i)}, \bar{\beta}_{X, j^{\prime}}^{\left(i^{\prime}\right)}\right]=0$ (if $\left.i \neq i^{\prime}\right)$;

$\left(R_{6}\right)\left[\bar{\alpha}_{X, j}^{(i)}, \bar{\beta}_{X, j^{\prime}}^{\left(i^{\prime}\right)}\right]= \begin{cases}\bar{\zeta}_{X, i}^{\left(i^{\prime}\right)} & \left(\text { if } j=j^{\prime} \text { and } i \neq i^{\prime}\right) \\ 0 & \left(\text { if } j \neq j^{\prime} \text { and } i \neq i^{\prime}\right)\end{cases}$

Proof. Assertion (i) follows from [12], Proposition 3.2, (i). Assertion (ii) follows from [8], Proposition 1. Assertion (iii) follows from [21], (2.8.2).

Lemma 4.1. Let $1 \leq i<j \leq r$ be integers.

(i) The following diagram induced by the cartesian diagram defining $U_{(r) \underline{\{i, j\}}}$ (cf. Definition 2.4, (i)) is cartesian:

$$
\begin{aligned}
& \operatorname{Gr}\left(\Delta_{\left.X_{(r)} \underline{\{i, j\}}\right)} \stackrel{\text { via } p_{U \frac{\{i, j\}}{X_{(r-1)}}: i}^{\longrightarrow}}{\operatorname{Gr}\left(\Delta_{X_{(r-1)}}\right)} \underset{\downarrow \text { via } p_{U_{X_{(r-2)}}: j-1}}{\downarrow \frac{\{i, j\}}{X_{(r-1)}: j \downarrow} \downarrow}\right. \\
& \operatorname{Gr}\left(\Delta_{X_{(r-1)}}\right) \underset{\text { via } p_{U_{X_{(r-1)}}: i}}{\longrightarrow} \operatorname{Gr}\left(\Delta_{X_{(r-2)}}\right) \text {. }
\end{aligned}
$$

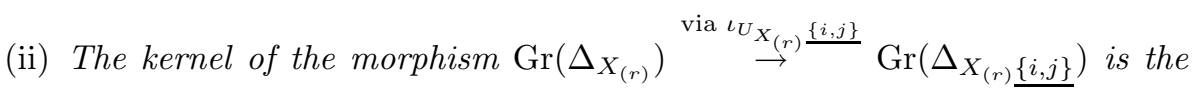
ideal generated by $\bar{\zeta}_{X, i}^{(j)}=\bar{\zeta}_{X, j}^{(i)}$ (cf. the statement of Proposition 4.1, (iii)). In particular, the set

$$
\left\{\bar{\zeta}_{X, i}^{(j)}\right\}
$$


is a base $\left(\right.$ over $\left.\mathbb{Q}_{l}\right)$ of the kernel of

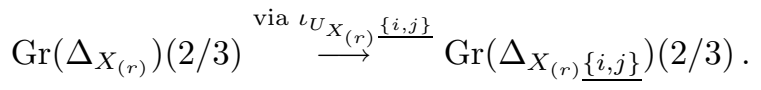

Proof. Assertion (i) follows from Lemma 2.3, together with Proposition 1.4, (ii). Assertion (ii) follows from the fact that the kernel of morphism $\operatorname{Gr}\left(\Delta_{X_{(r)} / X_{(r-1)}}^{(i)}\right) \rightarrow \operatorname{Gr}\left(\Delta_{X_{(r-1)} / X_{(r-2)}}^{(i)}\right)$ induced by the left-hand vertical arrow in the commutative diagram

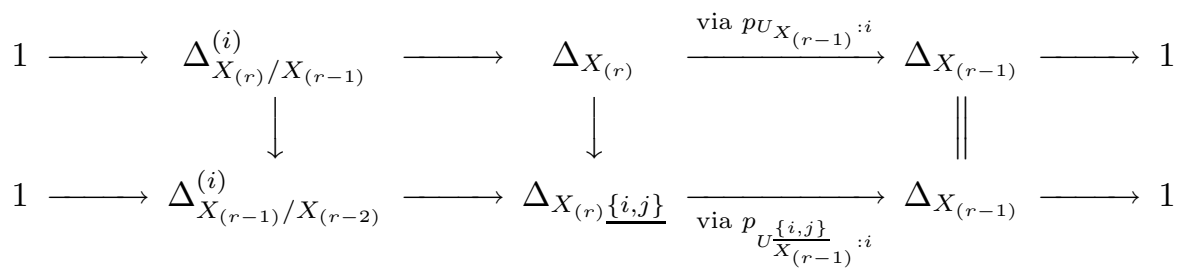

is the ideal generated by $\bar{\zeta}_{X, i}^{(j)}=\bar{\zeta}_{X, j}^{(i)}$. (This follows easily from an observation concerning the generators of the graded Lie algebras $\Delta_{X_{(r)} / X_{(r-1)}}^{(i)}$ and $\Delta_{X_{(r-1)} / X_{(r-2)}}^{(i)}$ given in [8], Proposition 1.)

Let $1 \leq i<j \leq r+1$ be integers. Next, let us fix choices of the inertia subgroups

$$
\mathfrak{I}_{X_{(r+1)}\{i, j\}} \subseteq \Pi_{X_{(r+1)}}
$$

(among the various conjugates of $\mathfrak{I}_{X_{(r+1)}\{i, j\}}$ ) for $1 \leq i<j \leq r+1$. (Note that, by Lemma 2.2, these choices induce choices of the subgroups

$$
\mathfrak{D}_{X_{(r+1)}\{i, j\}} \subseteq \Pi_{X_{(r+1)}} ;
$$

moreover, by considering the images of these subgroups via the surjection induced by $p_{X_{(r)}: k}^{\log }$ [where $1 \leq k \leq r+1$ ], these choices induce $r+1$ respective choices of the subgroups

$$
\mathfrak{I}_{X_{(r)}\{i, j\}} \subseteq \Pi_{X_{(r)}}
$$

and

$$
\left.\mathfrak{D}_{X_{(r)}\{i, j\}} \subseteq \Pi_{X_{(r)}} .\right)
$$

Lemma 4.2 (cf. [7], Lemma 4.3). Let $1 \leq i<j \leq r+1$ be integers. Let

$$
I_{\{i, j\}}=\left\{\begin{array}{ll}
\{i-1, j-1\} & (\text { if } i \neq 1) \\
\{1, j-1\} & \text { (if } i=1 \text { and } j \neq 2) \\
\{1,2\} & \text { (if } i=1 \text { and } j=2)
\end{array} \quad k_{\{i, j\}}=\left\{\begin{array}{l}
1(\text { if } i \neq 1) \\
2(\text { if } i=1 \text { and } j \neq 2) \\
3(\text { if } i=1 \text { and } j=2)
\end{array}\right.\right.
$$




$$
l_{\{i, j\}}=\left\{\begin{array}{l}
1(\text { if } i \neq 1) \\
2(\text { if } i=1)
\end{array} \quad m_{\{i, j\}}= \begin{cases}i-1 & (\text { if } i \neq 1) \\
1 & \text { (if } i=1)\end{cases}\right.
$$

Then the commutative diagram

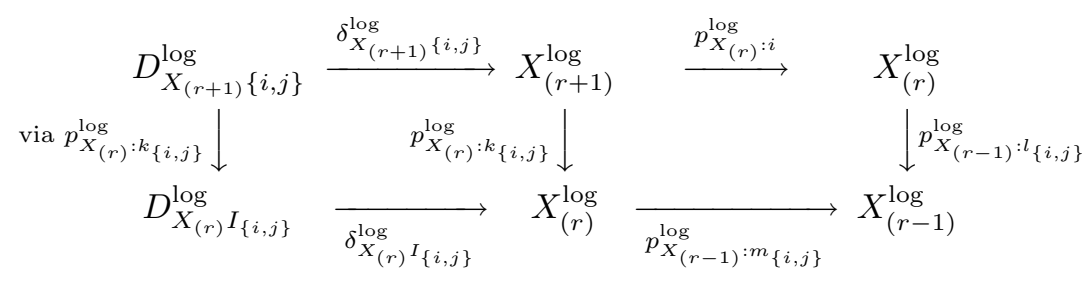

(cf. the discussion following Definition 2.2) induces the following cartesian diagram:

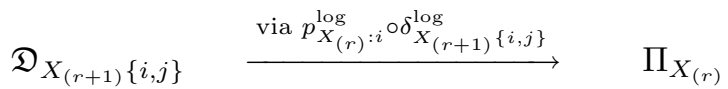

$$
\begin{aligned}
& \text { via } p_{X_{(r)}: k_{\{i, j\}} \downarrow}^{\log } \downarrow \\
& \downarrow \text { via } p_{X(r-1)}^{\log } l_{\{i, j\}} \\
& \mathfrak{D}_{X_{(r)} I_{\{i, j\}}} \underset{\operatorname{via} p_{X_{(r-1)}: m_{\{i, j\}}}^{\log } \circ \delta_{X_{(r)} I_{\{i, j\}}}^{\log }}{\Pi_{X_{(r-1)}}} .
\end{aligned}
$$

Proof. By the definitions, the commutative diagram

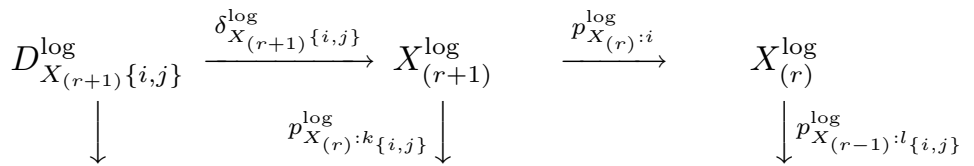

$$
\begin{aligned}
& D_{X_{(r)} I_{\{i, j\}}}^{\log } \underset{\delta_{X(r) I_{\{i, j\}}}^{\log }}{\longrightarrow} X_{(r)}^{\log } \underset{p_{X_{(r-1)}: m_{\{i, j\}}}^{\log }}{\longrightarrow} X_{(r-1)}^{\log }
\end{aligned}
$$

induces a commutative diagram

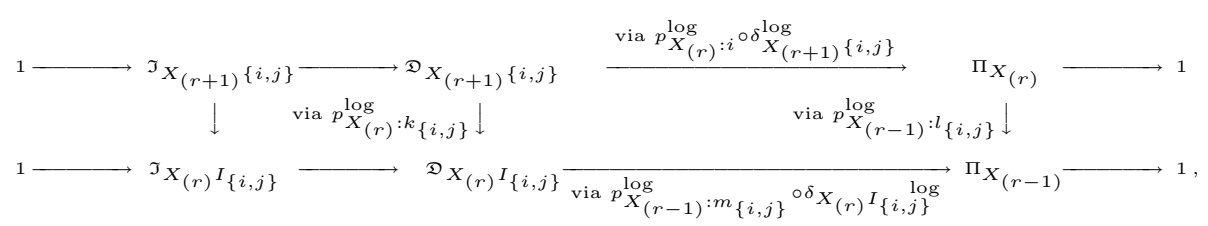

where the horizontal sequences are exact. Now since the restriction of the morphism $D_{X_{(r+1)}\{i, j\}}^{\log } \rightarrow D_{X_{(r)} I_{\{i, j\}}}^{\log }$ induced by $p_{X_{(r)}: k_{\{i, j\}}}^{\log }$ to the generic point of $D_{X_{(r+1)}\{i, j\}}^{\log }$ is strict, we conclude that the left-hand vertical arrow is an isomorphism. This completes the proof of Lemma 4.2 . 
Moreover, let us fix a section $s_{0}^{\prime}: G_{K} \rightarrow \Pi_{X_{(r)}}$ of the morphism $\Pi_{X_{(r)}} \rightarrow$ $G_{K}$ induced by the structure morphism of $U_{X_{(r)}}$ and a lifting $s_{0}$ of $s_{0}^{\prime}$ to $G_{K} \rightarrow$ $\mathfrak{D}_{X_{(r+1)}\{1,2\}}$, i.e., a morphism $G_{K} \rightarrow \mathfrak{D}_{X_{(r+1)}\{1,2\}}$ such that the composite of this morphism and $\mathfrak{D}_{X_{(r+1)}\{1,2\}} \hookrightarrow \Pi_{X_{(r+1)}} \stackrel{\text { via } x_{X(r): 1}^{\log }}{\rightarrow} \Pi_{X_{(r)}}$ coincides with $s_{0}^{\prime}$. Note that since $G_{K}$ is free, such a section and lifting always exist. Then the section $s_{0}$ of the natural morphism $\Pi_{X_{(r+1)}} \rightarrow G_{K}$ determines natural actions of $G_{K}$ (by conjugation) on $\Delta_{X_{(r+1)}}$, and on $\Delta_{X_{(r+1)} \underline{\{i, j\}}}$, hence also on

$$
\begin{gathered}
\operatorname{Lin}_{X_{(r+1)}}(a / b) \stackrel{\text { def }}{=} \operatorname{Lin}\left(\Delta_{X_{(r+1)}}(a / b)\right)\left(\mathbb{Q}_{l}\right) ; \\
\operatorname{Lin}_{X_{(r+1)} \underline{\{i, j\}}}(a / b) \stackrel{\text { def }}{=} \operatorname{Lin}\left(\Delta_{X_{(r+1)} \underline{\underline{i i, j\}}}}(a / b)\right)\left(\mathbb{Q}_{l}\right) ; \\
\operatorname{Lie}_{X_{(r+1)}}(a / b) \stackrel{\operatorname{def}}{=} \operatorname{Lie}\left(\Delta_{X_{(r+1)}}(a / b)\right) ; \operatorname{Lie}_{X_{(r+1)} \underline{\{i, j\}}}(a / b) \stackrel{\operatorname{def}}{=} \operatorname{Lie}\left(\Delta_{X_{(r+1)} \underline{\{i, j\}}}(a / b)\right) ; \\
\operatorname{Gr}_{\mathbb{Q}_{l}}\left(\Delta_{X_{(r+1)}}\right)(a / b) ; \operatorname{Gr}_{\mathbb{Q}_{l}}\left(\Delta_{X_{(r+1)} \underline{\underline{\{i, j\}}}}\right)(a / b)
\end{gathered}
$$

for $a, b \in \mathbb{Z}$ such that $1 \leq a \leq b$ (cf. Definition 1.1, (i)).

Proposition 4.2 (cf. [16], Proposition 3.2, (i), (ii)). $\quad$ Let $1 \leq i<j \leq$ $r+1$ be integers, and $a, b \in \mathbb{Z}$ such that $1 \leq a \leq b$.

(i) The eigenvalues of the action of $\operatorname{Fr}_{K}$ on $\operatorname{Lie}_{X_{(r+1)}}(a / a+1)$ (respectively, $\left.\operatorname{Lie}_{X_{(r+1)} \underline{\{i, j\}}}(a / a+1)\right)$ are algebraic numbers all of whose complex absolute values are equal to $q_{K}^{a / 2}$.

(ii) There is a unique $G_{K}$-equivariant isomorphism of Lie algebras

$$
\begin{gathered}
\operatorname{Lie}_{X_{(r+1)}}(a / b) \stackrel{\sim}{\longrightarrow} \operatorname{Gr}_{\mathbb{Q}_{l}}\left(\Delta_{X_{(r+1)}}\right)(a / b) \\
\left(\text { respectively, } \operatorname{Lie}_{X_{(r+1)} \underline{\{i, j\}}}(a / b) \stackrel{\sim}{\longrightarrow} \operatorname{Gr}_{\mathbb{Q}_{l}}\left(\Delta_{X_{(r+1)} \underline{\{i, j\}}}\right)(a / b)\right)
\end{gathered}
$$

which induces the identity isomorphism

$$
\operatorname{Lie}_{X_{(r+1)}}(c / c+1) \stackrel{\sim}{\longrightarrow} \operatorname{Gr}_{\mathbb{Q}_{l}}\left(\Delta_{X_{(r+1)}}\right)(c / c+1)
$$

(respectively, $\left.\operatorname{Lie}_{X_{(r+1)} \underline{\{i, j\}}}(c / c+1) \stackrel{\sim}{\longrightarrow} \operatorname{Gr}_{\mathbb{Q}_{l}}\left(\Delta_{X_{(r+1)}} \underline{\{i, j\}}\right)(c / c+1)\right)$

for all $c \in \mathbb{Z}$ such that $a \leq c \leq b-1$.

Proof. Assertion (i) follows immediately from the "Riemann hypothesis for abelian varieties over finite fields" (cf. e.g., [18], p. 206). Assertion (ii) follows formally from assertion (i) by considering the eigenspaces with respect to the action of $\operatorname{Fr}_{K}$. 


\section{Definition 4.2.}

(i) We shall write

$$
M_{X} \stackrel{\text { def }}{=} \operatorname{Hom}_{\mathbb{Z}_{l}}\left(H^{2}\left(\Delta_{X}, \mathbb{Z}_{l}\right), \mathbb{Z}_{l}\right)
$$

(cf. the discussion preceding [16], Remark 1). Note that $M_{X}$ is (noncanonically) isomorphic to $\mathbb{Z}_{l}(1)$ as a $G_{K}$-module, where "(1)" denotes a Tate twist.

(ii) Let $1 \leq i<j \leq r+1$ be integers. Then there exists a natural isomorphism $M_{X} \stackrel{\sim}{\rightarrow} \mathfrak{I}_{X_{(r+1)}\{i, j\}}$ (cf. [16], Proposition 1.5, (ii), (iii), also the statement of [16], Proposition 2.1). We shall denote this isomorphism by $m_{X_{(r+1)}\{i, j\}}$.

(iii) The cup product on the group cohomology of $\Delta_{X}$

$$
\bigwedge^{2} H^{1}\left(\Delta_{X}, M_{X}\right) \longrightarrow H^{2}\left(\Delta_{X}, M_{X} \otimes_{\mathbb{Z}_{l}} M_{X}\right)
$$

determines an isomorphism

$$
\operatorname{Hom}\left(\Delta_{X}^{\mathrm{ab}}, M_{X}\right) \stackrel{\sim}{\longrightarrow} \Delta_{X}^{\mathrm{ab}}
$$

hence a natural $G_{K}$-equivariant injection

$$
M_{X} \hookrightarrow \bigwedge^{2} \Delta_{X}^{\mathrm{ab}}
$$

(cf. the discussion preceding [16], Definition 3.2). We shall denote this $G_{K}$-equivariant injection by $i_{X}^{\text {cup }}$.

(iv) The isomorphism

$$
\operatorname{Hom}\left(\Delta_{X}^{\mathrm{ab}}, M_{X}\right) \stackrel{\sim}{\longrightarrow} \Delta_{X}^{\mathrm{ab}}
$$

in (iii) determines a homomorphism

$$
\bigwedge^{2} \Delta_{X}^{\mathrm{ab}} \longrightarrow M_{X}
$$

We shall denote by $a \cup_{X} a^{\prime}$ the image of $a \wedge a^{\prime}$ via this homomorphism, where $a, a^{\prime} \in \Delta_{X}^{\mathrm{ab}}$.

Proposition 4.3 (cf. [16], Proposition 3.2, (iii)). Let us write

$$
V_{U_{X_{(r+1)}}} \stackrel{\text { def }}{=} \bigoplus_{i<j}\left(\mathfrak{I}_{X_{(r+1)}\{i, j\}} \otimes_{\mathbb{Z}_{l}} \mathbb{Q}_{l}\right) \bigoplus \operatorname{Lie}_{X_{(r+1)}}(1 / 2)
$$


(Note that by applying the natural isomorphisms $m_{X_{(r+1)}\{i, j\}}: M_{X} \stackrel{\sim}{\rightarrow} \mathfrak{I}_{X_{(r+1)}\{i, j\}}$ and the identity morphism $\operatorname{Lie}_{X_{(r+1)}}(1 / 2) \stackrel{\sim}{\rightarrow}\left(\bigoplus_{k=1}^{r+1}\left(\Delta_{X}^{\mathrm{ab}}\right)_{k}\right) \otimes_{\mathbb{Z}_{l}} \mathbb{Q}_{l}\left[\right.$ where $\left(\Delta_{X}^{\mathrm{ab}}\right)_{k}$ is the copy of $\Delta_{X}^{\mathrm{ab}}$ indexed by $\left.k\right]$, one obtains a natural isomorphism of $V_{U_{X_{(r+1)}}}$ with the $\mathbb{Q}_{l}$-vector space obtained by tensoring the free $\mathbb{Z}_{l}$-module

$$
\bigoplus_{i<j} M_{X} \oplus \bigoplus_{k=1}^{r+1}\left(\Delta_{X}^{\mathrm{ab}}\right)_{k}
$$

with $\left.\mathbb{Q}_{l}.\right)$ Then the first isomorphism in Proposition 4.2 , (ii), together with the natural inclusions $\mathfrak{I}_{X_{(r+1)}\{i, j\}} \hookrightarrow \Delta_{X_{(r+1)}}$, determine a $G_{K^{-}}$equivariant morphism

$$
V_{U_{X}(r+1)} \longrightarrow \operatorname{Lie}_{X_{(r+1)}}(1 / \infty)
$$

which exhibits, in a $G_{K}$-equivariant fashion, $\operatorname{Lie}_{X_{(r+1)}}(1 / \infty)$ as the quotient of the completion with respect to the filtration topology of the free Lie algebra $\mathfrak{L i e}\left(V_{U_{X_{(r+1)}}}\right)$ generated by $V_{U_{X_{(r+1)}}}$ equipped with a natural grading, hence also a filtration, by taking the $\mathfrak{I}_{X_{(r+1)}\{i, j\}} \otimes_{\mathbb{Z}_{l}} \mathbb{Q}_{l}$ to be of weight $2, \operatorname{Lie}_{X_{(r+1)}}(1 / 2)$ to be of weight 1 , by the relations determined by the images of the morphisms

$$
\begin{aligned}
& \left(R_{1}^{\prime}\right) M_{X} \otimes_{\mathbb{Z}_{l}} \mathbb{Q}_{l}
\end{aligned}
$$

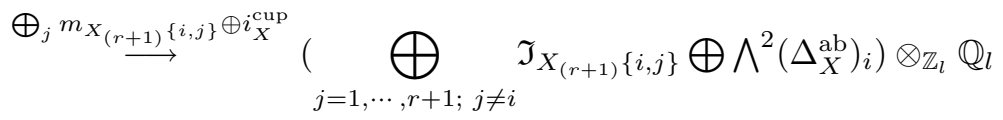

$$
\begin{aligned}
& \stackrel{\text { incl. } \oplus[,]}{\longrightarrow} \mathfrak{L i e}\left(V_{U_{X_{(r+1)}}}\right)(2 / 3) \quad(1 \leq i \leq r+1) ; \\
& \left(R_{3}^{\prime}\right)\left(\mathfrak{I}_{X_{(r+1)}\{i, k\}} \otimes_{\mathbb{Z}_{l}} \mathfrak{I}_{X_{(r+1)}\left\{i^{\prime}, k^{\prime}\right\}}\right) \otimes_{\mathbb{Z}_{l}} \mathbb{Q}_{l} \stackrel{[,]}{\longrightarrow} \mathfrak{L i e}\left(V_{U_{X_{(r+1)}}}\right)(4 / 5) \\
& \left(\{i, k\} \cap\left\{i^{\prime}, k^{\prime}\right\}=\emptyset\right) ; \\
& \left(R_{4}^{\prime}\right)\left(\mathfrak{I}_{X_{(r+1)}\{i, k\}} \otimes_{\mathbb{Z}_{l}}\left(\Delta_{X}^{\mathrm{ab}}\right)_{i^{\prime}}\right) \otimes_{\mathbb{Z}_{l}} \mathbb{Q}_{l} \stackrel{[,]}{\longrightarrow} \mathfrak{L i e}\left(V_{U_{X_{(r+1)}}}\right)(3 / 4) \\
& \left(i \neq i^{\prime}, \quad k \neq i^{\prime}\right) \text {; }
\end{aligned}
$$

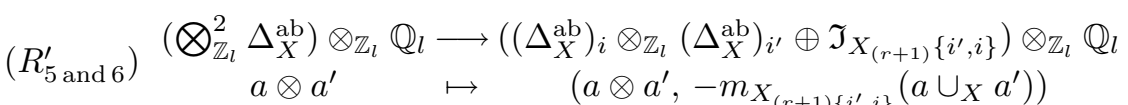

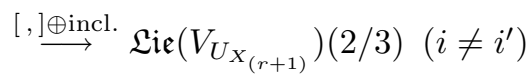

(cf. the relations in the statement of Proposition 4.1, (iii)), where "incl" is the natural inclusion, and ", ]" is the Lie bracket.

Proof. This follows from Propositions 4.1, (iii); 4.2, (ii). 
Definition 4.3 (cf. [16], Definition 3.3, (i)). $\quad$ Let $1 \leq i<j \leq r$ be integers, and $a, b \in \mathbb{Z}$ such that $1 \leq a \leq b$.

(i) Now we have natural $G_{K}$-equivariant surjections:

$\operatorname{Lin}_{X_{(r+1)}}(a / b) \rightarrow \operatorname{Lin}_{X_{(r+1)} \underline{\{1,2\}}}(a / b) ; \operatorname{Lie}_{X_{(r+1)}}(a / b) \rightarrow \operatorname{Lie}_{X_{(r+1)} \underline{\{1,2\}}}(a / b)$

(cf. the discussion preceding Proposition 4.2). We shall denote by

$$
\operatorname{Lin}_{X_{(r+1)}}^{\text {iner }}(a / b) ; \operatorname{Lie}_{X_{(r+1)}}^{\text {iner }}(a / b)
$$

the respective kernels of these surjections.

(ii) Now we have a natural $G_{K}$-equivariant morphism:

$$
\Delta_{X_{(r+1)} \underline{\{1,2\}}} \longrightarrow \operatorname{Lin}_{X_{(r+1)} \underline{\{1,2\}}}(1 / \infty)
$$

(cf. Definition 1.1, (ii)). We shall write

$$
\Delta_{X_{(r+1)}}^{\text {Lie }} \stackrel{\text { def }}{=} \Delta_{X_{(r+1)} \underline{\{1,2\}}} \times_{\operatorname{Lin}_{X_{(r+1)}} \underline{\{1,2\}}(1 / \infty)} \operatorname{Lin}_{X_{(r+1)}}(1 / \infty) .
$$

(We regard $\operatorname{Lin}_{X_{(r+1)} \underline{\{1,2\}}}(1 / \infty)$ and $\operatorname{Lin}_{X_{(r+1)}}(1 / \infty)$ as being equipped with the topology determined by the respective natural $l$-adic topologies of $\operatorname{Lin}_{X_{(r+1)}\{1,2\}}(1 / b)$ and $\operatorname{Lin}_{X_{(r+1)}}(1 / b)$ [where $b$ is a positive integer]; moreover, we regard $\Delta_{X_{(r+1)}}^{\mathrm{Lie}}$ as being equipped with the topology determined by the profinite topology of $\Delta_{X_{(r+1)} \underline{\{1,2\}}}$ and the topologies of $\operatorname{Lin}_{X_{(r+1)} \underline{\{1,2\}}}(1 / \infty)$ and $\operatorname{Lin}_{X_{(r+1)}}(1 / \infty)$.) Moreover, we shall denote by

$$
\operatorname{Int}_{X_{(r+1)}}^{\Delta}: \Delta_{X_{(r+1)}} \longrightarrow \Delta_{X_{(r+1)}}^{\text {Lie }}
$$

the $G_{K}$-equivariant morphism induced by the morphism

$$
\Delta_{X_{(r+1)}} \longrightarrow \Delta_{X_{(r+1)}} \underline{\{1,2\}}
$$

induced by $\iota_{U_{X_{(r+1)}} \underline{\{1,2\}}}$ (cf. Definition 2.4, (ii)) and the natural $G_{K^{-}}$ equivariant morphism

$$
\Delta_{X_{(r+1)}} \longrightarrow \operatorname{Lin}_{X_{(r+1)}}(1 / \infty) .
$$

(iii) Now we have a natural $G_{K}$-equivariant injection

$$
\begin{gathered}
\operatorname{Lin}_{X_{(r+1)}}^{\text {iner }}(b+1 / \infty) \stackrel{\sim}{\longrightarrow}\{1\} \times\{1\} \operatorname{Lin}_{X_{(r+1)}}^{\text {iner }}(b+1 / \infty) \\
\longrightarrow \Delta_{X_{(r+1)} \underline{\{1,2\}}} \times_{\operatorname{Lin}_{X_{(r+1)} \underline{\{1,2\}}}(1 / \infty)} \operatorname{Lin}_{X_{(r+1)}}(1 / \infty) \stackrel{\sim}{\longrightarrow} \Delta_{X_{(r+1)}}^{\mathrm{Lie}}
\end{gathered}
$$


whose image forms a closed normal subgroup of $\Delta_{X_{(r+1)}}^{\text {Lie }}$. We shall denote by

$$
\Delta_{X_{(r+1)}}^{\mathrm{Lie} \leq b}
$$

the quotient of $\Delta_{X_{(r+1)}}^{\text {Lie }}$ by this normal subgroup.

(iv) We shall write

$$
\Pi_{X_{(r+1)}}^{\text {Lie }} \stackrel{\text { def }}{=} \Delta_{X_{(r+1)}}^{\text {Lie }} \rtimes G_{K} ; \Pi_{X_{(r+1)}}^{\text {Lie } \leq b} \stackrel{\text { def }}{=} \Delta_{X_{(r+1)}}^{\text {Lie } \leq b} \rtimes G_{K},
$$

where the action of $G_{K}$ on $\Delta_{X_{(r+1)}}^{\text {Lie }}$ and $\Delta_{X_{(r+1)}}^{\text {Lie } \leq b}$ is the action induced by the section $s_{0}$ (cf. the discussion preceding Proposition 4.2). Moreover, we shall denote by

$$
\operatorname{Int}_{X_{(r+1)}}^{\Pi}: \Pi_{X_{(r+1)}} \longrightarrow \Pi_{X_{(r+1)}}^{\mathrm{Lie}}
$$

the morphism induced by $\operatorname{Int}_{X_{(r+1)}}^{\Delta}$.

(v) Now we have a natural morphism:

$$
\begin{aligned}
& \Pi_{X_{(r+1)}} \stackrel{\mathrm{Int}_{X_{(r+1)}}^{\Pi}}{\longrightarrow} \Pi_{X_{(r+1)}}^{\mathrm{Lie}} \rightarrow \Pi_{X_{(r+1)}}^{\mathrm{Lie} \leq b} \\
& \text { (respectively, } \mathfrak{D}_{X_{(r+1)}\{i, j\}} \hookrightarrow \Pi_{X_{(r+1)}} \stackrel{\operatorname{Int}_{X}^{\Pi}}{\longrightarrow} \Pi_{X_{(r+1)}}^{\mathrm{Lie}} \rightarrow \Pi_{X_{(r+1)}}^{\mathrm{Lie} \leq b} ; \\
& \text { respectively, } \left.\mathfrak{I}_{X_{(r+1)}\{i, j\}} \hookrightarrow \Pi_{X_{(r+1)}} \stackrel{\operatorname{Int}_{X_{(r+1)}}^{\Pi}}{\longrightarrow} \Pi_{X_{(r+1)}}^{\mathrm{Lie}} \rightarrow \Pi_{X_{(r+1)}}^{\mathrm{Lie} \leq b}\right) .
\end{aligned}
$$

We shall denote the image of this composite by

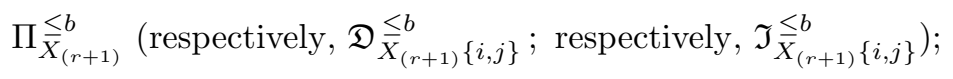

moreover, we shall write

$$
\begin{gathered}
\Delta_{X_{(r+1)}}^{\leq b} \stackrel{\text { def }}{=} \Pi_{X_{(r+1)}}^{\leq b} \cap \Delta_{X_{(r+1)}}^{\text {Lie } \leq b} ; \\
\mathfrak{D}_{X_{(r+1)}^{\Delta} \leq b}^{\Delta}\{i, j\} \stackrel{\text { def }}{=} \mathfrak{D}_{X_{(r+1)} \leq b}^{\leq b}\left\{\cap \Delta_{X_{(r+1)}}^{\text {Lie } \leq b}\right.
\end{gathered} .
$$

Proposition 4.4 (cf. [16], Proposition 3.2, (iv)). For each element $\alpha \in$ $\operatorname{Lin}_{X_{(r+1)}}(1 / \infty)$, there exists a unique element $\beta \in \operatorname{Lin}_{X_{(r+1)}}(1 / \infty)$ such that

$$
\operatorname{Fr}_{K} \circ \operatorname{Inn}(\alpha)=\operatorname{Inn}(\beta) \circ \operatorname{Fr}_{K} \circ \operatorname{Inn}\left(\beta^{-1}\right)
$$

(where "Inn $(-)$ " denotes the inner automorphism of $\operatorname{Lin}_{X_{(r+1)}}(1 / \infty)$ defined by conjugation by the element “(-)"). Moreover, when $\alpha$ lies in the subgroup obtained by tensoring the image of $\mathfrak{I}_{X_{(r+1)}\{1,2\}}$ via $\operatorname{Int}_{X_{(r+1)}}^{\Pi_{1}}$ with $\mathbb{Q}_{l}, \beta$ also lies in the subgroup obtained by tensoring the image of $\mathfrak{I}_{X_{(r+1)}\{1,2\}}$ via $\operatorname{Int}_{X_{(r+1)}}^{\Pi}$ with $\mathbb{Q}_{l}$. 
Proof. The second assertion of Proposition 4.4 follows immediately from the assumption on the section $s_{0}: G_{K} \rightarrow \Pi_{X_{(r+1)}}$ fixed in the discussion preceding Proposition 4.2 (note that the subgroup obtained by tensoring the image of $\mathfrak{I}_{X_{(r+1)}\{1,2\}}$ via $\operatorname{Int}_{X_{(r+1)}}^{\Pi}$ with $\mathbb{Q}_{l}$ is stable under the action of $\left.G_{K}\right)$; thus, we prove the first assertion of Proposition 4.4. For a positive integer $b$, we shall denote by $\alpha_{b}$ the image of $\alpha \in \operatorname{Lin}_{X_{(r+1)}}(1 / \infty)$ via the natural surjection $\operatorname{Lin}_{X_{(r+1)}}(1 / \infty) \rightarrow \operatorname{Lin}_{X_{(r+1)}}(1 / b)$. Then it is easily verified that to prove the first assertion of Proposition 4.4, it is enough to show that for any $b>2$, there exists $\beta \in \operatorname{Lin}_{X_{(r+1)}}(1 / b)$ such that $\alpha_{b}^{-1} \cdot \operatorname{Fr}_{K}(\beta) \cdot \beta^{-1} \in \operatorname{Lin}_{X_{(r)}}(b-1 / b)$. To verify this, we apply induction on $b$. The case where $b=2$ is immediate. Thus, assume that $b \geq 3$ and that there exists $\beta \in \operatorname{Lin}_{X_{(r+1)}}(1 / b-1)$ such that $\alpha_{b-1}^{-1} \cdot \operatorname{Fr}_{K}(\beta) \cdot \beta^{-1} \in \operatorname{Lin}_{X_{(r+1)}}(b-2 / b-1)$. Then there exist $\widetilde{\beta} \in \operatorname{Lin}_{X_{(r+1)}}(1 / b)$ and $\gamma \in \operatorname{Lin}_{X_{(r+1)}}(b-2 / b)$ such that $\alpha_{b}=\operatorname{Fr}_{K}(\widetilde{\beta}) \cdot \gamma \cdot \widetilde{\beta}^{-1}$. On the other hand, since it follows from Proposition 4.2, (i), that the morphism of $\mathbb{Q}_{l}$-vector spaces

$$
\begin{aligned}
\operatorname{Lin}_{X_{(r+1)}}(b-2 / b-1) & \longrightarrow \operatorname{Lin}_{X_{(r+1)}}(b-2 / b-1) \\
\delta & \mapsto \operatorname{Fr}_{K}(\delta) \cdot \delta^{-1}
\end{aligned}
$$

is an isomorphism, there exists $\delta \in \operatorname{Lin}_{X_{(r+1)}}(b-2 / b-1)$ such that $\gamma_{b-1}=$ $\operatorname{Fr}_{K}(\delta) \cdot \delta^{-1}$, where $\gamma_{b-1}$ is the image of $\gamma$ in $\operatorname{Lin}_{X_{(r+1)}}(b-2 / b-1)$. Therefore, there exists $\widetilde{\delta} \in \operatorname{Lin}_{X_{(r+1)}}(b-2 / b)$ such that

$$
\alpha_{b}^{-1} \cdot \operatorname{Fr}_{K}(\widetilde{\beta} \cdot \widetilde{\delta}) \cdot(\widetilde{\beta} \cdot \widetilde{\delta})^{-1} \in \operatorname{Lin}_{X_{(r+1)}}(b-1 / b) .
$$

This completes the proof of the first assertion of Proposition 4.4.

Remark 8 (cf. [16], Remark 35). Observe that changing the choice of a lifting

$$
s_{0}: G_{K} \longrightarrow \mathfrak{D}_{X_{(r+1)}}\{1,2\}
$$

of $s_{0}^{\prime}$ affects the image of the element $\operatorname{Fr}_{K} \in G_{K}$ via the composite of the inclusion $G_{K} \hookrightarrow \Pi_{X_{(r+1)}}$ with the morphism $\operatorname{Int}_{X_{(r+1)}}^{\Pi_{(r+1)}} \rightarrow \Pi_{X_{(r+1)}} \rightarrow \Pi_{X_{(x)}^{\mathrm{Lie}}}$ by conjugation by an element of the subgroup obtained by tensoring the image of $\mathfrak{I}_{X_{(r+1)}\{1,2\}}$ via $\operatorname{Int}_{X_{(r+1)}}^{\Pi}$ with $\mathbb{Q}_{l}$ (cf. Proposition 4.4). In particular, it follows that changing the choice of a lifting $G_{K} \rightarrow \mathfrak{D}_{X_{(r+1)}\{1,2\}}$ of $s_{0}^{\prime}$ affects the Galois invariant splitting of Proposition 4.2, (ii), by conjugation by an element of the subgroup obtained by tensoring the image of $\mathfrak{I}_{X_{(r+1)}\{1,2\}}$ via $\operatorname{Int}_{X_{(r+1)}}^{\Pi}$ with $\mathbb{Q}_{l}$. Put another way, if we identify the " $\operatorname{Lin}_{X_{(r+1)}}(1 / \infty) ", " \operatorname{Lin}_{X_{(r+1)}} \underline{\{1,2\}}(1 / \infty) "$ portion of $\Delta_{X_{(r+1)}}^{\text {Lie }}$ (cf. Definition 4.3 , (ii)) with the topological groups formed by the $\mathbb{Q}_{l}$-valued points of the pro-algebraic groups corresponding to the (completion of the) corresponding graded objects " $\operatorname{Gr}(-)(1 / \infty)$ " via the Galois invariant splitting of Proposition 4.2, (ii), then the following holds: 
Changing the choice of a lifting $s_{0}: G_{K} \rightarrow \mathfrak{D}_{X_{(r+1)}\{1,2\}}$ of $s_{0}^{\prime}$ affects the images of the morphism

$$
\operatorname{Int}_{X_{(r+1)}}^{\Pi}: \Pi_{X_{(r+1)}} \longrightarrow \Pi_{X_{(r+1)}}^{\mathrm{Lie}}
$$

by conjugation by an element of the subgroup obtained by tensoring the image of $\mathfrak{I}_{X_{(r+1)}\{1,2\}}$ via $\operatorname{Int}_{X_{(r+1)}}^{\Pi}$ with $\mathbb{Q}_{l}$.

Lemma 4.3. $\quad \operatorname{Int}_{X_{(r+1)}}^{\Delta}$ is an injection.

Proof. This follows from induction on $r$, Proposition 1.4, (ii), together with the fact that the central filtration

$$
\left\{\Delta_{X / S}(n)\right\}
$$

defined in the discussion following Lemma 1.1 satisfies that

$$
\bigcap_{n \geq 1} \Delta_{X / S}(n)=1
$$

Lemma 4.4. Let $r \geq 2$ be an integer. Then conjugates $\left(\right.$ in $\Delta_{X_{(r+1)}}^{\leq b}$ )

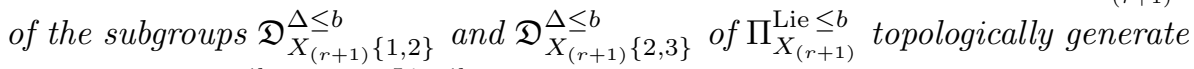
the subgroup $\Delta_{X_{(r+1)}}^{\leq b} \subseteq \Pi_{X_{(r+1)}}^{\text {Lie } \leq b}$.

Proof. This follows immediately from Proposition 2.4 and Lemma 4.3.

Lemma 4.5. Let $1 \leq i<j \leq r$ be integers, and $a, b \in \mathbb{Z}$ such that $1 \leq a \leq b$. Then the following hold:

(i) $\Delta_{X_{(r+1)}}^{\text {Lie } \leq 1}$ is naturally isomorphic to $\Delta_{X_{(r+1)}} \underline{\{1,2\}}$.

(ii) The kernel of the natural projection $\Pi_{X_{(r+1)}^{\mathrm{Lie}} \leq b+1}^{\mathrm{L}} \rightarrow \Pi_{X_{(r+1)}}^{\mathrm{Lie} \leq b}$ is isomorphic to

$$
\operatorname{Lin}_{X_{(r+1)}}^{\text {iner }}(b+1 / b+2) .
$$

In particular, the kernel of the natural projection $\mathfrak{D}_{X_{(r+1)} \leq b+1}\{i, j\} \rightarrow \mathfrak{D}_{X_{(r+1)}}^{\leq b}\{i, j\}$ is isomorphic to

$$
\begin{cases}1 & \text { if } b \neq 1 \text { or }\{i, j\} \neq\{1,2\}) \\ \mathfrak{I}_{X_{(r+1)}\{i, j\}} & \text { (if } b=1 \text { and }\{i, j\}=\{1,2\}) .\end{cases}
$$

Therefore, for $2 \leq b$, the natural projection $\mathfrak{D}_{X_{(r+1)} \leq b+1}^{\leq i, j\}} \rightarrow \mathfrak{D}_{X_{(r+1)}\{i, j\}}^{\leq b}$ is an isomorphism. 
Proof. This follows immediately from Lemma 4.2; Definition 4.3.

In the following, let us consider some assumptions on the section $s_{0}$ : $G_{K} \rightarrow \Pi_{X_{(r+1)}}$ fixed in the discussion preceding Proposition 4.2:

Definition 4.4. Let $r \geq 2$ be an integer. Then we shall say that the section $s_{0}: G_{K} \rightarrow \Pi_{X_{(r+1)}}$ (fixed in the discussion preceding Proposition 4.2) satisfies the condition $\left(\dagger_{\mathbb{P}}\right)$ (respectively, $\left(\dagger_{S}\right)$ for a set $S=\left\{x_{1}, \cdots, x_{r}\right\}$ of distinct $K$-rational points of $X$ of cardinality $r$ with an ordering) if the following holds:

The image of the section $s_{0}: G_{K} \rightarrow \Pi_{X_{(r+1)}}$ is included in

$$
\mathfrak{D}_{X_{(r+1)}\{1,2\}} \cap \mathfrak{D}_{X_{(r+1)}\{1,2,3\}} \subseteq \Pi_{X_{(r+1)}}
$$

(respectively, the section of the natural morphism $\Pi_{X_{(r)}} \rightarrow G_{K}$ ob-

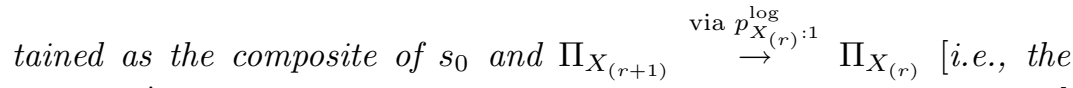
section $s_{0}^{\prime}: G_{K} \rightarrow \Pi_{X_{(r)}}$ in the discussion preceding Proposition 4.2] arises from the $K$-rational point of $U_{X_{(r)}}$ corresponding to $\left(x_{1}, \cdots, x_{r}\right)$ [thus, the image of the section $s_{0}: G_{K} \rightarrow \Pi_{X_{(r+1)}}$ is included in the decomposition subgroup of $\Pi_{X \backslash\left\{x_{1}, \cdots, x_{r}\right\}} \simeq \Pi_{X_{(r+1)}} \times_{\Pi_{X_{(r)}}} G_{K}$ associated to the cusp $x_{1}$ determined by the fixed decomposition subgroups $\left.\left.\mathfrak{D}_{X_{(r+1)}\{1,2\}} \subseteq \Pi_{X_{(r+1)}}\right]\right)$.

Note that since $G_{K}$ is free, and $D_{X_{(r+1)}\{1,2\}} \cap D_{X_{(r+1)}\{1,2,3\}}$ is non-empty, a section which satisfies the condition $\left(\dagger_{\mathbb{P}}\right)$ always exists.

By the discussion following Definition 2.2, we have an exact sequence

$$
1 \longrightarrow \mathfrak{I}_{X_{(r+1)}\{1,2,3\}} \longrightarrow \mathfrak{P}_{X_{(r+1)}\{1,2,3\}} \longrightarrow \Delta_{\mathbb{P}_{K}} \longrightarrow 1
$$

moreover, we also have a section of this sequence which is refered to the section of $\mathfrak{P}_{X_{(r+1)}\{1,2,3\}} \rightarrow \Delta_{\mathbb{P}_{K}}$ induced by $p_{X_{(r+1)}: i}^{\log }(i=1,2,3)$. Let us denote by $\Delta_{\mathbb{P}_{K}}\{i\}$ the image of the section of $\mathfrak{P}_{X_{(r+1)}}\{1,2,3\} \rightarrow \Delta_{\mathbb{P}_{K}}$ induced by $p_{X_{(r+1)}: i}^{\log }$. Note that the subgroup $\Delta_{\mathbb{P}_{K}}\{i\} \subseteq \mathfrak{D}_{X_{(r+1)}\{1,2,3\}}$ of $\mathfrak{D}_{X_{(r+1)}\{1,2,3\}}$ is normal by the definition of the section of $\mathfrak{P}_{X_{(r+1)}\{1,2,3\}} \rightarrow \Delta_{\mathbb{P}_{K}}$ induced by $p_{X_{(r+1)}: i}^{\log }$.

Definition 4.5. Since the subgroup

$$
\Delta_{\mathbb{P}_{K}}\{i\} \subseteq \mathfrak{D}_{X_{(r+1)}}\{1,2,3\}
$$


of $\mathfrak{D}_{X_{(r+1)}\{1,2,3\}}$ is normal, if the section $s_{0}$ satisfies the condition $\left(\dagger_{\mathbb{P}}\right)$ (cf. Definition 4.4), then the action of $G_{K}$ on $\mathfrak{D}_{X_{(r+1)}\{1,2,3\}}$ induced via conjugation induces an action of $G_{K}$ on $\Delta_{\mathbb{P}_{K}}\{i\}$. Therefore, we obtain a subgroup

$$
\Delta_{\mathbb{P}_{K}}\{i\} \rtimes G_{K} \subseteq \mathfrak{D}_{X_{(r+1)}\{1,2,3\}} .
$$

We shall write $\prod_{\mathbb{P}_{K}}\{i\} \stackrel{\text { def }}{=} \Delta_{\mathbb{P}_{K}}\{i\} \rtimes G_{K}$.

Lemma 4.6. The group $\prod_{\mathbb{P}_{K}}\{i\}$ is isomorphic to $\prod_{\mathbb{P}_{K}}$.

Proof. This follows immediately from the fact that the subgroup $\mathfrak{I}_{X_{(r+1)}\{1,2,3\}} \subseteq \mathfrak{D}_{X_{(r+1)}\{1,2,3\}}^{\Delta}$ of $\mathfrak{D}_{X_{(r+1)}\{1,2,3\}}^{\Delta}$ is included in the center of $\mathfrak{D}_{X_{(r+1)}}^{\Delta}\{1,2,3\}$, together with the fact that any element of the subgroup

$$
\Delta_{X_{(r-1)}} \times\{1\} \subseteq \Delta_{X_{(r-1)}} \times \Delta_{\mathbb{P}_{K}} \simeq \mathfrak{D}_{X_{(r+1)}\{1,2,3\}}^{\Delta} / \mathfrak{I}_{X_{(r+1)}\{1,2,3\}}
$$

of $\mathfrak{D}_{X_{(r+1)}\{1,2,3\}}^{\Delta} / \mathfrak{I}_{X_{(r+1)}\{1,2,3\}}$ commutes with any element of the subgroup $\{1\} \times \Delta_{\mathbb{P}_{K}} \subseteq \Delta_{X_{(r-1)}} \times \Delta_{\mathbb{P}_{K}} \simeq \mathfrak{D}_{X_{(r+1)}\{1,2,3\}}^{\Delta} / \mathfrak{I}_{X_{(r+1)}\{1,2,3\}}$ of $\mathfrak{D}_{X_{(r+1)}\{1,2,3\}}^{\Delta} /$ $\mathfrak{I}_{X_{(r+1)}\{1,2,3\}}$.

Definition 4.6 (cf. [16], Definitions 3.2; 3.3, (i)).

(i) We shall denote by

$$
\left\{\Delta_{\mathbb{P}_{K}}\{2\}(n)\right\}
$$

the central filtration of $\Delta_{\mathbb{P}_{K}}\{2\}$ with respect to the surjection

$$
\Delta_{\mathbb{P}_{K}}\{2\} \rightarrow 1
$$

(cf. Definition 1.1, (i)). Then it follows immediately from Lemma 2.1 that $\operatorname{Lin}\left(\Delta_{\mathbb{P}_{K}}\{2\}(2 / 3)\right)\left(\mathbb{Q}_{l}\right)$ is naturally isomorphic to

$$
\left(\mathfrak{I}_{X_{(r+1)}\{1,2\}} \oplus \mathfrak{I}_{X_{(r+1)}\{2,3\}}\right) \otimes_{\mathbb{Z}_{l}} \mathbb{Q}_{l} .
$$

Now we shall write

$$
\Delta_{\mathbb{P}_{K}}^{\mathrm{Lie}}\{2\} \stackrel{\text { def }}{=} \operatorname{Lin}\left(\Delta_{\mathbb{P}_{K}}\{2\}(1 / \infty)\right)\left(\mathbb{Q}_{l}\right) \times_{\left(\mathfrak{I}_{X_{(r+1)}\{2,3\}} \otimes_{\mathbb{Z}_{l}} \mathbb{Q}_{l}\right)} \mathfrak{I}_{X_{(r+1)}\{2,3\}},
$$

where the morphism $\operatorname{Lin}\left(\Delta_{\mathbb{P}_{K}}\{2\}(1 / \infty)\right)\left(\mathbb{Q}_{l}\right) \rightarrow \mathfrak{I}_{X_{(r+1)}\{2,3\}} \otimes_{\mathbb{Z}_{l}} \mathbb{Q}_{l}$ (respectively, $\left.\mathfrak{I}_{X_{(r+1)}\{2,3\}} \rightarrow \mathfrak{I}_{X_{(r+1)}\{2,3\}} \otimes_{\mathbb{Z}_{l}} \mathbb{Q}_{l}\right)$ is the composite

$$
\operatorname{Lin}\left(\Delta_{\mathbb{P}_{K}}\{2\}(1 / \infty)\right)\left(\mathbb{Q}_{l}\right) \rightarrow \operatorname{Lin}\left(\Delta_{\mathbb{P}_{K}}\{2\}(2 / 3)\right)\left(\mathbb{Q}_{l}\right)
$$




$$
\stackrel{\sim}{\longrightarrow}\left(\mathfrak{I}_{X_{(r+1)}\{1,2\}} \oplus \mathfrak{I}_{X_{(r+1)}\{2,3\}}\right) \otimes_{\mathbb{Z}_{l}} \mathbb{Q}_{l} \stackrel{\mathrm{pr}_{2}}{\rightarrow} \mathfrak{I}_{X_{(r+1)}\{2,3\}} \otimes_{\mathbb{Z}_{l}} \mathbb{Q}_{l}
$$

(respectively, the natural inclusion). Then by the definition of $\Delta_{X_{(r+1)}}^{\mathrm{Lie}}$, the natural morphism $\operatorname{Lin}\left(\Delta_{\mathbb{P}_{K}}\{2\}(1 / \infty)\right)\left(\mathbb{Q}_{l}\right) \rightarrow \operatorname{Lin}_{X_{(r+1)}}(1 / \infty)$ (induced by $\left.\Delta_{\mathbb{P}_{K}}\{2\} \hookrightarrow \Delta_{X_{(r+1)}}\right)$ and the the natural inclusion $\mathfrak{I}_{X_{(r+1)}\{2,3\}} \hookrightarrow$ $\Delta_{X_{(r+1)} \underline{\{1,2\}}}$ induce a natural morphism

$$
\Delta_{\mathbb{P}_{K}}^{\mathrm{Lie}}\{2\} \longrightarrow \Delta_{X_{(r+1)}}^{\mathrm{Lie}}
$$

Now let us assume that the section $s_{0}$ fixed in the discussion preceding Proposition 4.2 satisfies the condition $\left(\dagger_{\mathbb{P}}\right)$ (cf. Definition 4.4). Then we shall write

$$
\prod_{\mathbb{P}_{K}}^{\text {Lie }}\{2\} \stackrel{\text { def }}{=} \Delta_{\mathbb{P}_{K}}^{\text {Liee }}\{2\} \rtimes G_{K},
$$

where the action of $G_{K}$ on $\Delta_{\mathbb{P}_{K}}^{\text {Lie }}\{2\}$ is the action obtained via conjugation. Now it follows that the above morphism $\Delta_{\mathbb{P}_{K}}^{\text {Lie }}\{2\} \rightarrow \Delta_{X_{(r+1)}}^{\text {Lie }}$ induces a morphism

$$
\Pi_{\mathbb{P}_{K}}^{\mathrm{Lie}}\{2\} \longrightarrow \Pi_{X_{(r+1)}}^{\mathrm{Lie}}
$$

moreover, the following diagram commutes

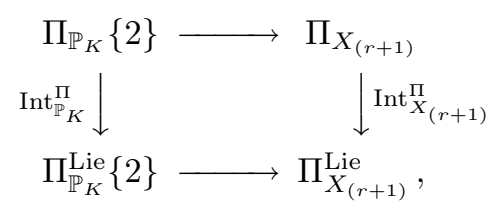

where the left-hand vertical arrow $\operatorname{Int}_{\mathbb{P}_{K}}^{\Pi}$ is the morphism obtained by a similar way to the way to define $\operatorname{Int}_{X_{(r+1)}}^{\Pi}$.

(ii) Let $S=\left\{x_{1}, \cdots, x_{r}\right\} \subseteq X(K)$ be a set of distinct $K$-rational points of $X$ of cardinality $r$ with an ordering. Then we shall denote by $U_{S} \subseteq X$ (respectively, $U_{\underline{S}} \subseteq X$ ) the open subscheme obtained as the complement of $S$ (respectively, $\underline{S} \stackrel{\text { def }}{=} S \backslash\left\{x_{1}\right\}$ ) in $X$, and by

$$
\left.\left\{\Delta_{U_{S}}(n)\right\} \text { (respectively, }\left\{\Delta_{U_{\underline{S}}}(n)\right\}\right)
$$

the central filtration of the pro-l fundamental group $\Delta_{U_{S}}$ (respectively, $\Delta_{U_{\underline{S}}}$ ) of $U_{S}$ (respectively, $U_{\underline{S}}$ ) with respect to the natural surjection

$$
\Delta_{U_{S}} \rightarrow \Delta_{X}^{\mathrm{ab}} \text { (respectively, } \Delta_{U_{\underline{S}}} \rightarrow \Delta_{X}^{\mathrm{ab}} \text { ). }
$$

Now we shall write

$$
\Delta_{U_{S}}^{\text {Lie }} \stackrel{\text { def }}{=} \operatorname{Lin}\left(\Delta_{U_{S}}(1 / \infty)\right)\left(\mathbb{Q}_{l}\right) \times_{\operatorname{Lin}\left(\Delta_{U_{\underline{S}}}(1 / \infty)\right)\left(\mathbb{Q}_{l}\right)} \Delta_{U_{\underline{S}}},
$$


where the morphism implicit in the fiber product $\Delta_{U_{\underline{S}}} \rightarrow \operatorname{Lin}\left(\Delta_{U_{\underline{S}}}(1 / \infty)\right)\left(\mathbb{Q}_{l}\right)$ is the morphism considered in Definition 1.1, (ii). Let us denote by $\mathfrak{D}_{x_{1}}$ the decomposition subgroup associated to $x_{1}$ of the geometrically pro- $l$ fundamental group $\Pi_{U_{S}}$ of $U_{S}$ (well-defined, up to conjugate). Then $\mathfrak{D}_{x_{1}}$ fits into an exact sequence

$$
1 \longrightarrow \mathfrak{I}_{x_{1}} \longrightarrow \mathfrak{D}_{x_{1}} \longrightarrow G_{K} \longrightarrow 1
$$

where $\mathfrak{I}_{x_{1}}$ is the inertia subgroup associated to $x_{1}$ of $\Pi_{U_{S}}$ (well-defined, up to conjugate). Let us fix a section $s_{0}^{S}: G_{K} \rightarrow \mathfrak{D}_{x_{1}}$ of this exact sequence. Then we obtain actions of $G_{K}$ on $\Pi_{U_{S}}$, and on the geometrically pro-l fundamental group $\Pi_{U_{\underline{S}}}$ of $U_{\underline{S}}$ (via conjugation), hence also on

$$
\begin{gathered}
\operatorname{Lin}\left(\Delta_{U_{S}}(a / b)\right)\left(\mathbb{Q}_{l}\right) ; \operatorname{Lin}\left(\Delta_{U_{\underline{S}}}(a / b)\right)\left(\mathbb{Q}_{l}\right) ; \\
\operatorname{Lie}\left(\Delta_{U_{S}}(a / b)\right) ; \operatorname{Lie}\left(\Delta_{U_{\underline{S}}}(a / b)\right) ; \\
\operatorname{Gr}_{\mathbb{Q}_{l}}\left(\Delta_{U_{S}}(a / b)\right) ; \operatorname{Gr}_{\mathbb{Q}_{l}}\left(\Delta_{U_{\underline{S}}}(a / b)\right) ; \Delta_{U_{S}}^{\text {Lie }}
\end{gathered}
$$

for $a, b \in \mathbb{Z}$ such that $1 \leq a \leq b$. Then we shall write

$$
\Pi_{U_{S}}^{\text {Lie }} \stackrel{\text { def }}{=} \Delta_{U_{S}}^{\text {Lie }} \rtimes G_{K} .
$$

Proposition 4.5 (cf. [16], Proposition 3.2, (iii)).

(i) If the section $s_{0}$ satisfies the condition $\left(\dagger_{\mathbb{P}}\right)$, then there exsits a unique $G_{K}$-equivariant isomorphism of Lie algebras

$$
\operatorname{Lie}\left(\Delta_{\mathbb{P}_{K}}\{2\}(a / b)\right) \stackrel{\sim}{\longrightarrow} \operatorname{Gr}_{\mathbb{Q}_{l}}\left(\Delta_{\mathbb{P}_{K}}\{2\}\right)(a / b)
$$

(where $a \leq b$ are integers) which induces the identity morphism

$$
\operatorname{Lie}\left(\Delta_{\mathbb{P}_{K}}\{2\}(c / c+1)\right) \stackrel{\sim}{\longrightarrow} \operatorname{Gr}_{\mathbb{Q}_{l}}\left(\Delta_{\mathbb{P}_{K}}\{2\}\right)(c / c+1)
$$

for all $c \in \mathbb{Z}$ such that $a \leq c \leq b-1$. Now let us write

$$
V_{\mathbb{P}_{K}}\{2\} \stackrel{\text { def }}{=}\left(\Im_{X_{(r+1)}\{1,2\}} \oplus \mathfrak{I}_{X_{(r+1)}\{2,3\}}\right) \otimes_{\mathbb{Z}_{l}} \mathbb{Q}_{l} .
$$

(Note that, by applying the natural isomorphisms $m_{X_{(r+1)}\{i, j\}}: M_{X} \stackrel{\sim}{\rightarrow}$ $\mathfrak{I}_{X_{(r+1)}\{i, j\}}[c f$. Definition 4.2, (ii)], one obtains a natural isomorphism of $V_{\mathbb{P}_{K}}\{2\}$ with the $\mathbb{Q}_{l}$-vector space obtained by tensoring the free $\mathbb{Z}_{l}$-module

$$
M_{X} \oplus M_{X}
$$


with $\left.\mathbb{Q}_{l}.\right)$ Then the natural inclusions $\mathfrak{I}_{X_{(r+1)}\{i, j\}} \hookrightarrow \Delta_{\mathbb{P}_{K}}\{2\}$ (where $\{i, j\}=$ $\{1,2\},\{2,3\})$, together with the above $G_{K}$-equivariant isomorphism of Lie algebras

$$
\operatorname{Lie}\left(\Delta_{\mathbb{P}_{K}}\{2\}(a / b)\right) \stackrel{\sim}{\longrightarrow} \operatorname{Gr}_{\mathbb{Q}_{l}}\left(\Delta_{\mathbb{P}_{K}}\{2\}\right)(a / b)
$$

determine a $G_{K}$-equivariant morphism

$$
V_{\mathbb{P}_{K}}\{2\} \longrightarrow \operatorname{Lie}\left(\Delta_{\mathbb{P}_{K}}\{2\}(1 / \infty)\right)
$$

which exhibits, in a $G_{K}$-equivariant fashion, $\operatorname{Lie}\left(\Delta_{\mathbb{P}_{K}}\{2\}(1 / \infty)\right)$ as the completion with respect to the filtration topology of the free Lie algebra $\mathfrak{L i e}\left(V_{\mathbb{P}_{K}}\{2\}\right)$ generated by $V_{\mathbb{P}_{K}}\{2\}$ equipped with a natural grading, hence also a filtration, by taking the $\mathfrak{I}_{X_{(r+1)}\{i, j\}} \otimes_{\mathbb{Z}_{l}} \mathbb{Q}_{l}$ to be of weight 2 . Moreover, the morphism of Lie algebras $\operatorname{Lie}\left(\Delta_{\mathbb{P}_{K}}\{2\}(1 / \infty)\right) \rightarrow \operatorname{Lie}_{X_{(r+1)}}(1 / \infty)$ corresponding to the morphism $\operatorname{Lin}\left(\Delta_{\mathbb{P}_{K}}\{2\}(1 / \infty)\right)\left(\mathbb{Q}_{l}\right) \rightarrow \operatorname{Lin}_{X_{(r+1)}}(1 / \infty)\left(\mathbb{Q}_{l}\right)$ discussed in Definition 4.6, (i), coincides with the morphism induced by the natural inclusion

$$
V_{\mathbb{P}_{K}}\{2\} \hookrightarrow V_{U_{X_{(r+1)}}}
$$

(cf. Proposition 4.3).

(ii) Let $S=\left\{x_{1}, \cdots, x_{r}\right\} \subseteq X(K)$ be a set of distinct $K$-rational points of $X$ of cardinality $r$ equipped with an ordering. Then there exists a unique $G_{K}$-equivariant isomorphism of Lie algebras

$$
\begin{gathered}
\operatorname{Lie}\left(\Delta_{U_{S}}(a / b)\right) \stackrel{\sim}{\longrightarrow} \operatorname{Gr}_{\mathbb{Q}_{l}}\left(\Delta_{U_{S}}\right)(a / b) \\
\left(\text { respectively, } \operatorname{Lie}\left(\Delta_{U_{\underline{S}}}(a / b)\right) \stackrel{\sim}{\longrightarrow} \operatorname{Gr}_{\mathbb{Q}_{l}}\left(\Delta_{U_{\underline{S}}}\right)(a / b)\right)
\end{gathered}
$$

(where $a \leq b$ are integers) which induces the identity morphism

$$
\begin{gathered}
\operatorname{Lie}\left(\Delta_{U_{S}}(c / c+1)\right) \stackrel{\sim}{\longrightarrow} \operatorname{Gr}_{\mathbb{Q}_{l}}\left(\Delta_{U_{S}}\right)(c / c+1) \\
\left(\text { respectively, } \operatorname{Lie}\left(\Delta_{U_{\underline{S}}}(c / c+1)\right) \stackrel{\sim}{\longrightarrow} \operatorname{Gr}_{\mathbb{Q}_{l}}\left(\Delta_{U_{\underline{S}}}\right)(c / c+1)\right)
\end{gathered}
$$

for all $c \in \mathbb{Z}$ such that $a \leq c \leq b-1$. Now let us write

$$
V_{U_{S}} \stackrel{\text { def }}{=} \bigoplus_{1 \leq i \leq r}\left(M_{X} \otimes_{\mathbb{Z}_{l}} \mathbb{Q}_{l}\right) \bigoplus \operatorname{Lie}\left(\Delta_{U_{S}}\right)(1 / 2)
$$

(respectively, $\left.V_{U_{\underline{S}}} \stackrel{\text { def }}{=} \bigoplus_{2 \leq i \leq r}\left(M_{X} \otimes_{\mathbb{Z}_{l}} \mathbb{Q}_{l}\right) \bigoplus \operatorname{Lie}\left(\Delta_{U_{S}}\right)(1 / 2)\right)$. 
$\left[\right.$ Note that, by applying the identity morphism $\operatorname{Lie}\left(\Delta_{U_{S}}\right)(1 / 2) \stackrel{\sim}{\rightarrow} \Delta_{X}^{\mathrm{ab}} \otimes_{\mathbb{Z}_{l}} \mathbb{Q}_{l}$ $\left(\right.$ respectively, $\left.\operatorname{Lie}\left(\Delta_{U_{\underline{S}}}\right)(1 / 2) \stackrel{\sim}{\rightarrow} \Delta_{X}^{\mathrm{ab}} \otimes_{\mathbb{Z}_{l}} \mathbb{Q}_{l}\right)$, one obtains a natural isomorphism of $V_{U_{S}}$ (respectively, $V_{U_{\underline{S}}}$ ) with the $\mathbb{Q}_{l}$-vector space obtained by tensoring the free $\mathbb{Z}_{l}$-module

$$
\bigoplus_{1 \leq i \leq r} M_{X} \bigoplus \Delta_{X}^{\mathrm{ab}} \quad \text { (respectively, } \bigoplus_{2 \leq i \leq r} M_{X} \bigoplus \Delta_{X}^{\mathrm{ab}} \text { ) }
$$

with $\mathbb{Q}_{l}$.] Then the above $G_{K}$-equivariant isomorphism

$$
\operatorname{Lie}\left(\Delta_{U_{S}}(a / b)\right) \stackrel{\sim}{\longrightarrow} \operatorname{Gr}_{\mathbb{Q}_{l}}\left(\Delta_{U_{S}}\right)(a / b)
$$

(respectively, $\left.\operatorname{Lie}\left(\Delta_{U_{\underline{S}}}(a / b)\right) \stackrel{\sim}{\longrightarrow} \operatorname{Gr}_{\mathbb{Q}_{l}}\left(\Delta_{U_{\underline{S}}}\right)(a / b)\right)$,

together with the composite of the natural isomorphism $M_{X} \stackrel{\sim}{\rightarrow} \mathfrak{I}_{x_{i}}\left[U_{S}\right]$ (respectively, $M_{X} \stackrel{\sim}{\rightarrow} \mathfrak{I}_{x_{i}}\left[U_{\underline{S}}\right]$ ) (cf. Definition 4.2, (ii)) and the natural inclusions $\mathfrak{I}_{x_{i}}\left[U_{S}\right] \hookrightarrow \Delta_{U_{S}}$ (respectively, $\mathfrak{I}_{x_{i}}\left[U_{\underline{S}}\right] \hookrightarrow \Delta_{U_{\underline{S}}}$ ) [where $\mathfrak{I}_{x_{i}}\left[U_{S}\right]$ (respectively, $\mathfrak{I}_{x_{i}}\left[U_{\underline{S}}\right]$ ) is the inertia subgroup of $\Delta_{U_{S}}$ (respectively, $\Delta_{U_{\underline{S}}}$ ) associated to $x_{i} \in S$ (respectively, $x_{i} \in \underline{S}$ )], determine a $G_{K}$-equivariant morphism

$$
\begin{gathered}
V_{U_{S}} \longrightarrow \operatorname{Lie}\left(\Delta_{U_{S}}(1 / \infty)\right) \\
\left(\text { respectively, } V_{U_{\underline{S}}} \longrightarrow \operatorname{Lie}\left(\Delta_{U_{\underline{S}}}(1 / \infty)\right)\right)
\end{gathered}
$$

which exhibits, in a $G_{K}$-equivariant fashion, $\operatorname{Lie}\left(\Delta_{U_{S}}(1 / \infty)\right)$ (respectively, $\left.\operatorname{Lie}\left(\Delta_{U_{\underline{S}}}(1 / \infty)\right)\right)$ as the quotient of the completion with respect to the filtration topology of the free Lie algebra $\mathfrak{L i e}\left(V_{U_{S}}\right)$ (respectively, $\mathfrak{L i e}\left(V_{U_{\underline{S}}}\right)$ ) generated by $V_{U_{S}}$ (respectively, $V_{U_{\underline{S}}}$ ) equipped with a natural grading, hence also a filtration, by taking the $M_{X} \otimes_{\mathbb{Z}_{l}} \mathbb{Q}_{l}$ to be of weight $2, \operatorname{Lie}\left(\Delta_{U_{S}}(1 / 2)\right)$ (respectively, $\left.\operatorname{Lie}\left(\Delta_{U_{\underline{S}}}(1 / 2)\right)\right)$ to be of weight 1 , by the relations determined by the image of the morphism:

$$
\begin{aligned}
& M_{X} \otimes_{\mathbb{Z}_{l}} \mathbb{Q}_{l} \stackrel{\oplus \operatorname{id}_{M_{X}}}{\rightarrow} \stackrel{\text { cup }}{\text { cup }}\left(\bigoplus_{i=1}^{r} M_{X} \bigoplus \bigwedge^{2} \Delta_{X}^{\mathrm{ab}}\right) \otimes_{\mathbb{Z}_{l}} \mathbb{Q}_{l} \stackrel{\text { incl. } \oplus[,]}{\rightarrow} \mathfrak{L i e}\left(V_{U_{S}}\right)(2 / 3) \\
& \text { (respectively, } M_{X} \otimes_{\mathbb{Z}_{l}} \mathbb{Q}_{l} \stackrel{\oplus \operatorname{id}_{M_{X}} \oplus i_{X}^{\text {cup }}}{\longrightarrow}\left(\bigoplus_{i=2}^{r} M_{X} \bigoplus \bigwedge^{2} \Delta_{X}^{\mathrm{ab}}\right) \otimes_{\mathbb{Z}_{l}} \mathbb{Q}_{l} \\
& \left.\stackrel{\text { incl. }}{\rightarrow}[,] \mathfrak{L i e}\left(V_{U_{\underline{S}}}\right)(2 / 3)\right),
\end{aligned}
$$

where "incl" is the natural inclusion, and "I, ]" is the Lie bracket. 
Proof. This follows from [16], Propositions 3.3; 3.4.

In the following, let $L$ be a finite field whose characteristic (respectively, cardinality) we denote by $p_{L}$ (respectively, $q_{L}$ ) such that $l$ is invertible in $L$ (i.e., $\left.l \neq p_{L}\right), \bar{L}$ an algebraic closure of $L, G_{L} \stackrel{\text { def }}{=} \operatorname{Gal}(\bar{L} / L)$, and $Y$ a proper hyperbolic curve over $L$. Moreover, let $\alpha_{(r)}: \Pi_{X_{(r)}} \stackrel{\sim}{\rightarrow} \Pi_{Y_{(r)}}$ be a Frobeniuspreserving (cf. Definition 2.5) and order-preserving (cf. Definition 2.6, (ii)) isomorphism, and $t_{0}^{\prime}: G_{L} \rightarrow \Pi_{Y_{(r)}}$ the section of the natural morphism $\Pi_{Y_{(r)}} \rightarrow$ $G_{L}$ corresponding to $s_{0}^{\prime}$ under the isomorphism $\alpha_{(r)}$.

Lemma 4.7. Let $\alpha_{(r)}: \Pi_{X_{(r)}} \stackrel{\sim}{\rightarrow} \Pi_{Y_{(r)}}$ be a Frobenius-preserving and order-preserving isomorphism. Then, for any integer $1 \leq r^{\prime} \leq r+1$, there exists a unique isomorphism

$$
\begin{gathered}
\alpha_{\left(r^{\prime}\right)}^{\mathrm{Lie}}: \Pi_{X_{\left(r^{\prime}\right)}}^{\mathrm{Lie}} \stackrel{\sim}{\longrightarrow} \Pi_{Y_{\left(r^{\prime}\right)}}^{\mathrm{Lie}} \\
\left(\text { respectively, } \alpha_{\left(r^{\prime}\right)}^{\mathrm{Lie} \leq b}: \Pi_{X_{\left(r^{\prime}\right)}}^{\mathrm{Lie} \leq b} \stackrel{\sim}{\longrightarrow} \Pi_{Y_{\left(r^{\prime}\right)}}^{\mathrm{Lie} \leq b} \text { for any } b \geq 1\right)
\end{gathered}
$$

which, for any integer $1 \leq i \leq r^{\prime}$, fits into commutative diagrams

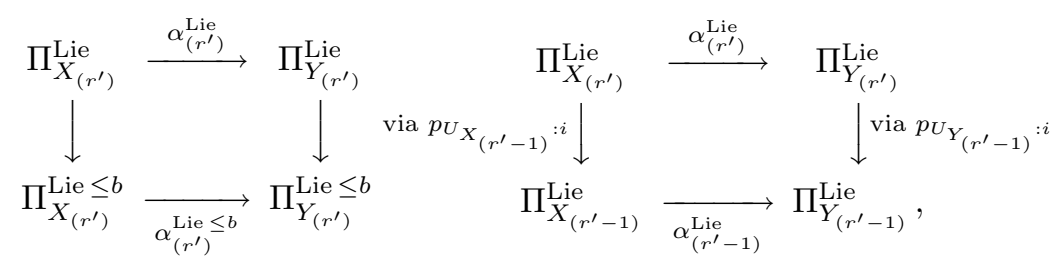

and, if $r^{\prime} \leq r$, then $\alpha_{\left(r^{\prime}\right)}^{\text {Lie }}$ fits into a commutative diagram

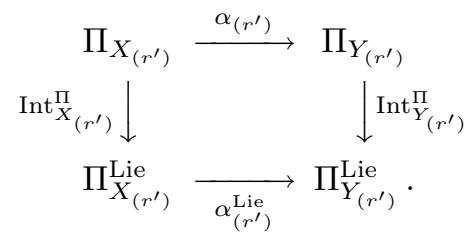

Proof. By the discussion following Definition 2.6, $\alpha_{(r)}$ induces a Frobeniuspreserving isomorphism $\alpha: \Pi_{X} \stackrel{\sim}{\rightarrow} \Pi_{Y}$. Thus, it follows from Proposition 4.3 that we obtain an isomorphism $\operatorname{Lie}_{X_{\left(r^{\prime}\right)}}(1 / \infty) \stackrel{\sim}{\rightarrow} \operatorname{Lie}_{Y_{\left(r^{\prime}\right)}}(1 / \infty)$; therefore, by the functoriality of "Lin", we obtain an isomorphism

$$
\alpha_{\left(r^{\prime}\right)}^{\mathrm{Lie}}: \Pi_{X_{\left(r^{\prime}\right)}}^{\mathrm{Lie}} \stackrel{\sim}{\longrightarrow} \Pi_{Y_{\left(r^{\prime}\right)}}^{\mathrm{Lie}}
$$

(respectively, $\left.\alpha_{\left(r^{\prime}\right)}^{\mathrm{Lie} \leq b}: \Pi_{X_{\left(r^{\prime}\right)}^{\mathrm{Lie}} \leq b}^{\mathrm{\sim}} \stackrel{\sim}{\longrightarrow} \Pi_{Y_{\left(r^{\prime}\right)}}^{\mathrm{Lie} \leq b}\right)$. 
By constructions, it follows that the diagrams

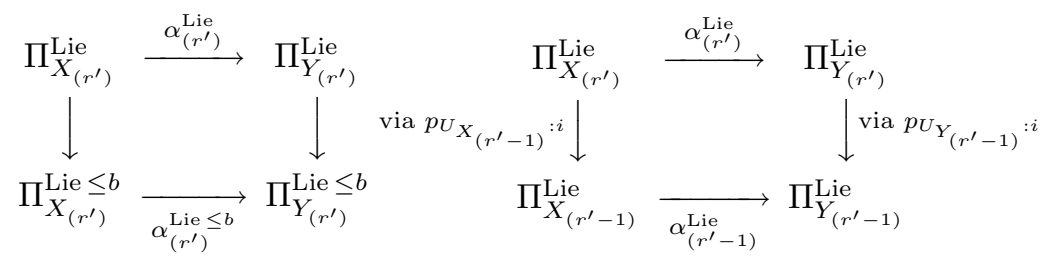

commute.

Assume that $r^{\prime} \leq r$. Then the isomorphism $\alpha_{\left(r^{\prime}\right)}: \Pi_{X_{\left(r^{\prime}\right)}} \stackrel{\sim}{\rightarrow} \Pi_{Y_{\left(r^{\prime}\right)}}$ (obtained in the discussion following Definition 2.6) induces an isomorphism $\Pi_{X_{\left(r^{\prime}\right)}}^{\mathrm{Lie}} \stackrel{\sim}{\rightarrow} \Pi_{Y_{\left(r^{\prime}\right)}}^{\mathrm{Lie}}$ which fits into the commutative diagram

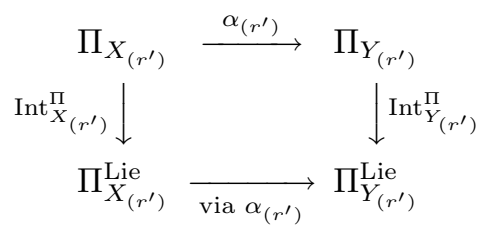

by the definitions of $\Pi_{X_{\left(r^{\prime}\right)}}^{\mathrm{Lie}}$ and $\Pi_{Y_{\left(r^{\prime}\right)}}^{\mathrm{Lie}}$. Thus, to prove Lemma 4.7, it is enough to show that this isomorphism of $\Pi_{X_{\left(r^{\prime}\right)}^{\mathrm{Lie}}}^{\mathrm{L}}$ with $\Pi_{Y_{\left(r^{\prime}\right)}}^{\mathrm{Lie}}$ coincides with the isomorphism $\alpha_{\left(r^{\prime}\right)}^{\text {Lie }}$. On the other hand, this follows from Proposition 4.2, (ii), by considering the eigenspaces with respect to the action of the Frobenius element.

By Lemma 4.7, we obtain an isomorphism

$$
\alpha_{(r+1)}^{\mathrm{Lie}}: \Pi_{X_{(r+1)}}^{\mathrm{Lie}} \stackrel{\sim}{\longrightarrow} \Pi_{Y_{(r+1)}}^{\mathrm{Lie}} .
$$

Note that by the construction of $\alpha_{(r+1)}^{\mathrm{Lie}}$, together with the assumption on the section $s_{0}$ which is fixed in the discussion preceding Proposition 4.2, we may assume that

$\alpha_{(r+1)}^{\text {Lie }}$ maps the image of $\mathfrak{I}_{X_{(r+1)}\{1,2\}}$ via $\operatorname{Int}_{X_{(r+1)}}^{\Pi}$ bijectively onto the image of $\mathfrak{I}_{Y_{(r+1)}\{1,2\}}$ via $\operatorname{Int}_{Y_{(r+1)}}^{\Pi}$.

Lemma 4.8. Let $r \geq 2$ be an integer. Then if the section $s_{0}$ satisfies the condition $\left(\dagger_{\mathbb{P}}\right)$, the following conditions are equivalent:

(i) $\alpha_{(r)}$ is tripod-preserving.

(ii) The isomorphism $\alpha_{(r+1)}^{\text {Lie }}$ maps the image of $\Pi_{\mathbb{P}_{K}}\{2\}$ via $\operatorname{Int}_{X_{(r+1)}}^{\Pi_{1}}$ bijectively onto the image of $\Pi_{\mathbb{P}_{L}}\{2\}$ via $\operatorname{Int}_{Y_{(r+1)}}^{\Pi_{(1)}}$. 
In particular, if $\alpha_{(r)}$ is tripod-preserving, then $\alpha_{(r+1)}^{\mathrm{Lie}}$ maps the image (via $\operatorname{Int}_{X_{(r+1)}}^{\Pi}$ ) of the decomposition subgroup $\mathfrak{D}_{X_{(r+1)}}^{\mathbb{P}}\{2,3\}$ of $\Pi_{\mathbb{P}_{K}}\{2\}$ such that $\mathfrak{D}_{X_{(r+1)} \mathbb{P}_{(2,3\}}} \cap \Delta_{X_{(r+1)}}$ coincides with $\mathfrak{I}_{X_{(r+1)}\{2,3\}}$ bijectively onto a $\Pi_{Y_{(r+1)}}$ conjugate of the image (via $\operatorname{Int}_{Y_{(r+1)}}^{\Pi}$ ) of the decomposition subgroup $\mathfrak{D}_{Y_{(r+1)}}^{\mathbb{P}}\{2,3\}$ of $\Pi_{\mathbb{P}_{L}}\{2\}$ such that $\mathfrak{D}_{Y_{(r+1)}^{\mathbb{P}}\{2,3\}} \cap \Delta_{Y_{(r+1)}}$ coincides with $\mathfrak{I}_{Y_{(r+1)}\{2,3\}}$.

Proof. It follows immediately from the definition of the term "tripodpreserving" that condition (ii) implies condition (i) (cf. Lemma 4.6). We prove the assertion that condition (i) implies condition (ii). Since $\alpha_{(r)}$ is tripodpreserving, there exists an isomorphism $\alpha_{\mathbb{P}}: \Pi_{\mathbb{P}_{K}}\{2\} \stackrel{\sim}{\rightarrow} \Pi_{\mathbb{P}_{L}}\{2\}$ such that the composite $M_{X}^{\oplus 2} \stackrel{\sim}{\rightarrow} M_{Y}^{\oplus 2}$ of the natural isomorphism $M_{X}^{\oplus 2} \stackrel{\sim}{\rightarrow} \Delta_{\mathbb{P}_{K}}^{\text {ab }}$ (cf. Definition 4.2, (ii)), the isomorphism $\Delta_{\mathbb{P}_{K}}^{\mathrm{ab}} \stackrel{\sim}{\rightarrow} \Delta_{\mathbb{P}_{L}}^{\mathrm{ab}}$ induced by $\alpha_{\mathbb{P}}$, and the natural isomorphism $\Delta_{\mathbb{P}_{L}}^{\mathrm{ab}} \stackrel{\sim}{\rightarrow} M_{L}^{\oplus 2}$ coincides with the isomorphism obtained by the isomorphism $M_{X} \rightarrow M_{Y}$ obtained by $\alpha_{(r)}$; moreover, it follows from the definitions of $\prod_{\mathbb{P}_{K}}^{\mathrm{Lie}}\{2\}$ and $\prod_{\mathbb{P}_{L}}^{\mathrm{Lie}}\{2\}$ that $\alpha_{\mathbb{P}}$ induces an isomorphism $\alpha_{\mathbb{P}}^{\mathrm{Lie}}$ : $\prod_{\mathbb{P}_{K}}^{\text {Lie }}\{2\} \stackrel{\sim}{\rightarrow} \Pi_{\mathbb{P}_{L}}^{\text {Lie }}\{2\}$ which fits into a commutative diagram

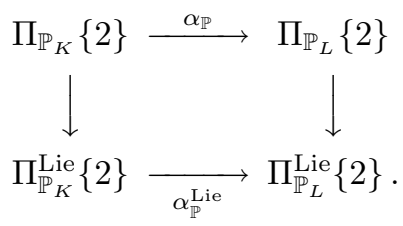

On the other hand, by Proposition 4.5, (i), the isomorphism $M_{X} \stackrel{\sim}{\rightarrow} M_{Y}$ induced by $\alpha_{(r)}$ induces an isomorphism $\prod_{\mathbb{P}_{K}}^{\mathrm{Lie}}\{2\} \stackrel{\sim}{\rightarrow} \prod_{\mathbb{P}_{L}}^{\mathrm{Lie}}\{2\}$ which fits into a commutative diagram

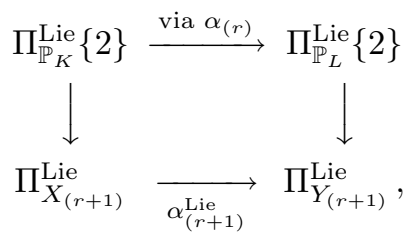

where the vertical arrows are the morphism obtained in Definition 4.6, (i). Thus, to prove Lemma 4.8, it is enough to show that this isomorphism of $\prod_{\mathbb{P}_{K}}^{\text {Liee }}\{2\}$ with $\prod_{\mathbb{P}_{L}}^{\text {Lie }}\{2\}$ (induced by $\alpha_{(r)}$ ) coincides with the isomorphism $\alpha_{\mathbb{P}}^{\text {Lie }}$. On the other hand, this follows from the fact that $\operatorname{Lie}\left(\Delta_{\mathbb{P}_{K}}\{2\}(1 / \infty)\right)$ (respectively, $\left.\operatorname{Lie}\left(\Delta_{\mathbb{P}_{L}}\{2\}(1 / \infty)\right)\right)$ is generated by the image of $V_{\mathbb{P}_{K}}\{2\}$ (respectively, $\left.V_{\mathbb{P}_{L}}\{2\}\right)$, by considering the eigenspaces with respect to the action of the respective Frobenius elements (cf. Proposition 4.5, (i)). 
Lemma 4.9. Let $S=\left\{x_{1}, \cdots, x_{r}\right\}$ be a set of distinct $K$-rational points of $X$ of cardinality $r$ with an ordering, $s_{S}: G_{K} \rightarrow \Pi_{X_{(r)}}$ the section of the natural morphism $\Pi_{X_{(r)}} \rightarrow G_{K}$ corresponding to the $K$-rational point $\left(x_{1}, \cdots, x_{r}\right) \in$ $U_{X_{(r)}}(K)$, and $\left(y_{1}, \cdots, y_{r}\right) \in U_{Y_{(r)}}(L)$ an L-rational point of $U_{Y_{(r)}}$ to which the section $t_{T}$, obtained as the composite $G_{L} \stackrel{\alpha_{(0)}^{-1}}{\rightarrow} G_{K} \stackrel{s_{S}}{\rightarrow} \Pi_{X_{(r)}} \stackrel{\alpha_{(r)}}{\rightarrow} \Pi_{Y_{(r)}}$, of the natural morphism $\Pi_{Y_{(r)}} \rightarrow G_{L}$ corresponds (cf. Proposition 2.5, (ii)). Let us write $T \stackrel{\text { def }}{=}\left\{y_{1}, \cdots, y_{r}\right\}$. Let $\alpha_{S, T}: \Pi_{U_{S}} \stackrel{\sim}{\rightarrow} \Pi_{V_{T}}$ be an isomorphism of the geometrically pro-l fundamental group $\Pi_{U_{S}}$ of $U_{S} \stackrel{\text { def }}{=} X \backslash S$ with the geometrically pro-l fundamental group $\Pi_{V_{T}}$ of $V_{T} \stackrel{\text { def }}{=} Y \backslash T$ such that the isomorphism $\Pi_{X} \stackrel{\sim}{\rightarrow} \Pi_{Y}$ induced by $\alpha_{S, T}$ coincides with the isomorphism $\alpha_{(1)}: \Pi_{X} \stackrel{\sim}{\rightarrow} \Pi_{Y}$ induced by $\alpha_{(r)}$. Let us assume that the section $s_{0}^{\prime}$ (respectively, $t_{0}^{\prime}$ ) of the natural morphism $\Pi_{X_{(r)}} \rightarrow G_{K}$ (respectively, $\Pi_{Y_{(r)}} \rightarrow G_{L}$ ) (fixed in the discussion preceding Proposition 4.2) coincides with $s_{S}$ (respectively, $t_{T}$ ). [In particular, the section $G_{K} \rightarrow \Pi_{X_{(r+1)}}$ (respectively, $G_{L} \rightarrow \Pi_{Y_{(r+1)}}$ ) fixed in the discussion preceding Proposition 4.2 satisfies the condition $\left(\dagger_{S}\right)$ (respectively, $\left.\left(\dagger_{T}\right)\right)$.] Then there exist morphisms $\Pi_{U_{S}} \rightarrow \Pi_{X_{(r+1)}^{\mathrm{Lie}}}^{\mathrm{L}}$ and $\Pi_{V_{T}} \rightarrow \Pi_{Y_{(r+1)}^{\mathrm{Lie}}}^{\mathrm{Lie}}$ which fit into a commutative diagram

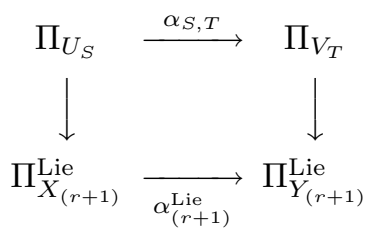

and satisfy the following condition: The quotient of $\Pi_{U_{S}}$ (respectively, $\Pi_{V_{T}}$ ) determined by the composite

$$
\begin{gathered}
\Pi_{U_{S}} \longrightarrow \Pi_{X_{(r+1)}}^{\mathrm{Lie}} \rightarrow \Pi_{X_{(r+1)} \underline{\{1,2\}}} \\
\text { (respectively, } \left.\Pi_{V_{T}} \longrightarrow \Pi_{Y_{(r+1)}}^{\mathrm{Lie}} \rightarrow \Pi_{Y_{(r+1)} \underline{\{1,2\}}}\right)
\end{gathered}
$$

coincides with the natural quotient $\Pi_{U_{S}} \rightarrow \Pi_{U_{\underline{S}}}$ (respectively, $\Pi_{V_{T}} \rightarrow \Pi_{V_{\underline{T}}}$ ), where $\underline{S} \stackrel{\text { def }}{=}\left\{x_{2}, \cdots, x_{r}\right\}$ (respectively, $\left.\underline{T} \stackrel{\text { def }}{=}\left\{y_{2}, \cdots, y_{r}\right\}\right)$; moreover, this composite determines an isomorphism $\Pi_{U_{\underline{S}}} \stackrel{\sim}{\rightarrow} \Pi_{X_{(r+1)} \underline{\{1,2\}}} \times_{\Pi_{X_{(r)}}} G_{K}$ (respectively, $\left.\Pi_{V_{\underline{T}}} \stackrel{\sim}{\rightarrow} \Pi_{Y_{(r+1)} \underline{\{1,2\}}} \times_{\Pi_{Y_{(r)}}} G_{L}\right)$, where the morphism $\Pi_{X_{(r+1)} \underline{\{1,2\}}} \rightarrow \Pi_{X_{(r)}}$ (respectively, $\Pi_{Y_{(r+1)} \underline{\{1,2\}}} \rightarrow \Pi_{Y_{(r)}}$ ) is the morphism induced by $\bar{U}_{(r+1)} \underline{\{1,2\}}$ $\stackrel{p_{U \frac{\{1,2\}}{X_{(r)}}: 2}^{\rightarrow}}{\longrightarrow} U_{X_{(r)}}$ (respectively, $\left.U_{Y_{(r+1)} \underline{\{1,2\}}} \stackrel{p^{U \frac{\{1,2\}}{Y_{(r)}}}: 2}{\rightarrow} U_{Y_{(r)}}\right)$, and the morphism $G_{K} \rightarrow$

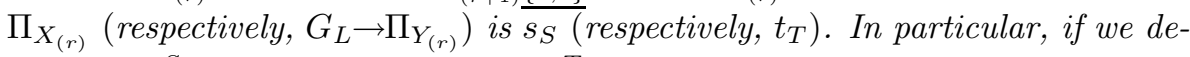
note by $\mathfrak{D}_{X_{(r+1)}\{2,3\}}^{S}$ (respectively, $\mathfrak{D}_{Y_{(r+1)}^{T}\{2,3\}}^{T}$ ) the decomposition subgroup of 
$\Pi_{U_{S}}$ (respectively, $\Pi_{V_{T}}$ ) associated to $x_{2}$ (respectively, $\left.y_{2}\right)$ such that $\mathfrak{D}_{X_{(r+1)}}^{S}\{2,3\}$ $\cap \Delta_{X_{(r+1)}}$ (respectively, $\mathfrak{D}_{Y_{(r+1)}^{T}\{2,3\}} \cap \Delta_{Y_{(r+1)}}$ ) coincides wtih $\mathfrak{I}_{X_{(r+1)}\{2,3\}}$

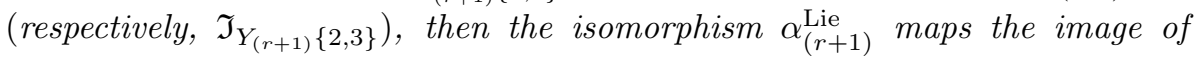
$\mathfrak{D}_{X_{(r+1)}\{2,3\}}$ via $\operatorname{Int}_{X_{(r+1)}}^{\Pi_{1}}$ bijectively onto a $\Pi_{Y_{(r+1)}}$-conjugate of the image of $\mathfrak{D}_{Y_{(r+1)}}^{T}\{2,3\}$ via $\operatorname{Int}_{X_{(r+1)}}^{\Pi}$.

Proof. By the assumption on $\alpha_{S, T}, \alpha_{S, T}$ induces an isomorphism $\alpha_{\underline{S}, \underline{T}}$ : $\Pi_{U_{\underline{S}}} \stackrel{\sim}{\rightarrow} \Pi_{V_{\underline{T}}}$. On the other hand, by the definitions of $\Pi_{X_{(r+1)} \underline{\{1,2\}}}$ and $\Pi_{Y_{(r+1)} \underline{\{1,2\}}}$, the isomorphism $\alpha_{(r)}: \Pi_{X_{(r)}} \stackrel{\sim}{\rightarrow} \Pi_{Y_{(r)}}$ induces an isomorphism $\Pi_{X_{(r+1)} \underline{\{1,2\}}} \times_{\Pi_{X_{(r)}}} G_{K} \stackrel{\sim}{\rightarrow} \Pi_{Y_{(r+1)} \underline{\{1,2\}}} \times_{\Pi_{Y_{(r)}}} G_{L}$, where these fiber products are as in the statement of Lemma 4.9 ; moreover, it follows from the assumption on $s_{S}$ (respectively, $t_{T}$ ) that the profinite group $\Pi_{X_{(r+1)} \underline{\{1,2\}}} \times_{\Pi_{X_{(r)}}} G_{K}$ (respectively, $\Pi_{Y_{(r+1)} \underline{\{1,2\}}} \times_{\Pi_{Y_{(r)}}} G_{L}$ ) is isomorphic to the geometrically pro- $l$ fundamental group of $U_{\underline{S}}$ (respectively, $V_{\underline{T}}$ ). Let us fix isomorphisms $\Pi_{U_{\underline{S}}} \stackrel{\sim}{\rightarrow}$ $\Pi_{X_{(r+1)} \underline{\{1,2\}}} \times_{\Pi_{X_{(r)}}} G_{K}$ and $\Pi_{V_{\underline{T}}} \stackrel{\sim}{\rightarrow} \Pi_{Y_{(r+1)} \underline{\{1,2\}}} \times_{\Pi_{Y_{(r)}}} G_{L}$. Then it follows from Proposition 4.6 below that by composition with a cuspidally inner automorphism of $\Pi_{Y_{(r+1)} \underline{\{1,2\}}} \times_{\Pi_{Y_{(r)}}} G_{L}$ (relative to $\Pi_{Y_{(r+1)} \underline{\{1,2\}}} \times_{\Pi_{Y_{(r)}}} G_{L} \rightarrow \Pi_{Y}$ ) if necessary, we may assume that the following diagram commutes:

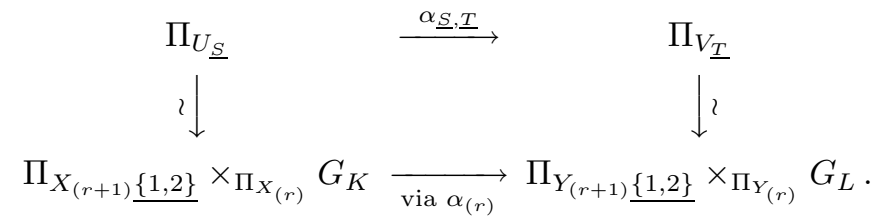

In particular, it follows from Proposition 4.5, (ii), together with the assumption that the isomorphism of $\Pi_{X} \stackrel{\sim}{\rightarrow} \Pi_{Y}$ induced by $\alpha_{S, T}$ coincides with the isomorphism $\alpha_{(1)}$, that we obtain a commutative diagram

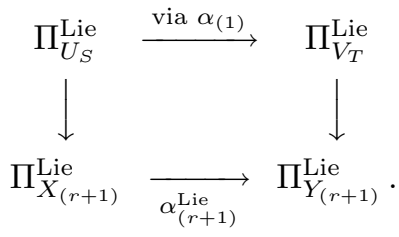

On the other hand, by the definitions of $\Pi_{U_{S}}^{\mathrm{Lie}}$ and $\Pi_{V_{T}}^{\mathrm{Lie}}, \alpha_{S, T}$ induces an isomor- 
phism $\alpha_{S, T}^{\mathrm{Lie}}: \Pi_{U_{S}}^{\mathrm{Lie}} \stackrel{\sim}{\rightarrow} \Pi_{V_{T}}^{\mathrm{Lie}}$ which fits into the following commutative diagram:

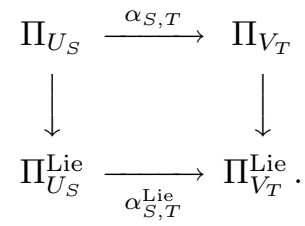

Therefore, to prove Lemma 4.9, it is enough to show that the isomorphism $\Pi_{U_{S}}^{\mathrm{Lie}} \stackrel{\sim}{\rightarrow} \Pi_{V_{T}}^{\mathrm{Lie}}$ induced by $\alpha_{(1)}$ coincides with the isomorphism $\alpha_{S, T}^{\mathrm{Lie}}: \Pi_{U_{S}}^{\mathrm{Lie}} \stackrel{\sim}{\rightarrow}$ $\Pi_{V_{T}}^{\mathrm{Lie}}$. On the other hand, this follows from a similar argument to the argument used in the proof of Lemma 4.8, together with Proposition 4.5, (ii).

Proposition 4.6. $\quad$ Let $S$ be a locally noetherian normal scheme which is geometrically connected over the finite filed $K, f: X \rightarrow S$ a family of hyperbolic curves such that $f \otimes_{K} \bar{K}: X \otimes_{K} \bar{K} \rightarrow S \otimes_{K} \bar{K}$ is of pro-l-exact type (cf. Definition 1.2), $s: S \rightarrow X$ a section of $f, U \subseteq X$ the open subscheme of $X$ obtained as the complement of the (scheme-theoretic) image of $s$, and $f^{U}: U \rightarrow S$ the restriction of $f$ to $U$. Let

$$
\alpha: \Pi_{U} \stackrel{\sim}{\longrightarrow} \Pi_{U}
$$

be an automorphism of the geometrically pro-l fundamental group $\Pi_{U}$ of $U$ which fits into a commutative diagram

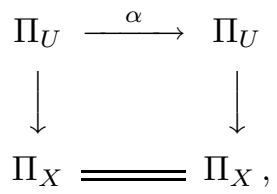

where $\Pi_{X}$ is the geometrically pro-l fundamental group of $X$, and the vertical arrows are the surjections induced by the natural open immersion $U \hookrightarrow X$. Then $\alpha$ is a cuspidally inner automorphism, i.e., there exists an element $\gamma$ of the kernel of the natural surjection $\Pi_{U} \rightarrow \Pi_{X}$ such that $\alpha=\operatorname{Inn}(\gamma)$.

Proof. If $S$ is isomorphic to the spectrum of a finite extension field of $K$, then Proposition 4.6 follows from a similar argument to the argument used in the proof of the uniqueness of " $\alpha_{\infty}$ " in [16], Theorem 3.1. Therefore, Proposition 4.6 follows from Lemma 4.10 below, together with the slimness of the kernel of the surjection induced by $f^{U}$ on geometrically pro- $l$ fundamental groups. (Note that it follows from Proposition 1.2, together with the assumption that $f \otimes_{K} \bar{K}: X \otimes_{K} \bar{K} \rightarrow S \otimes_{K} \bar{K}$ is of pro-l-exact type, that $f^{U} \otimes_{K} \bar{K}: U \otimes_{K} \bar{K} \rightarrow S \otimes_{K} \bar{K}$ is also of pro-l-exact type.) 
Lemma 4.10. Let

$$
1 \longrightarrow \Delta \longrightarrow \Pi \longrightarrow G \longrightarrow 1
$$

be an exact sequence of profinite groups, and $\phi$ an automorphism of $\Pi$ which induces the identity morphisms of $\Delta$ and $G$. Assume that $\Delta$ is slim (where we refer to the discussion entitled "Groups" in Introduction concerning the term "slim"). Then $\phi$ is the identity morphism.

Proof. By the slimness of $\Delta$, we have a natural isomorphism

$$
\Pi \stackrel{\sim}{\longrightarrow} \operatorname{Aut}(\Delta) \times_{\mathrm{Out}(\Delta)} G,
$$

where the morphism implicit in the fiber product $G \rightarrow \operatorname{Out}(\Delta)$ is the morphism induced by the natural morphism $\Pi \rightarrow \operatorname{Aut}(\Delta)$, together with the exact sequence in the statement of Lemma 4.10. Now it is easily verified that if $\phi$ is an automorphism of $\Pi$ which preserves the subgroup $\Delta \subseteq \Pi$, then the automorphism of $\operatorname{Aut}(\Delta) \times_{\operatorname{Out}(\Delta)} G$ corresponding to $\phi$ (under the above isomorphism) is given by

$$
\begin{aligned}
\operatorname{Aut}(\Delta) \times \times_{\operatorname{Out}(\Delta)} G & \stackrel{\sim}{\longrightarrow} \operatorname{Aut}(\Delta) \times \times_{\operatorname{Out}(\Delta)} G \\
(f, g) & \mapsto\left(\left.\left.\phi\right|_{\Delta} \circ f \circ \phi^{-1}\right|_{\Delta}, \bar{\phi}(g)\right),
\end{aligned}
$$

where $\bar{\phi}$ is the automorphism of $G$ induced by $\phi$. Thus, the assertion is immediate.

In the following, we assume that

$$
r \geq 2
$$

Lemma 4.11. The image of the diagonal morphism

$$
\Pi_{X_{(r)}} \longrightarrow \Pi_{X_{(r)}} \times_{\Pi_{X_{(r-1)}}} \Pi_{X_{(r)}} \stackrel{\sim}{ } \Pi_{X_{(r+1)} \underline{\{1,2\}}}
$$

(cf. Lemma 2.3) is a conjugate of $\mathfrak{D}_{X_{(r+1)}^{\leq 1}\{1,2\}}$ in $\Pi_{X_{(r+1)} \underline{\{1,2\}}} \simeq \Pi_{X_{(r+1)}}^{\mathrm{Lie} \leq 1}$ (cf. Lemma 4.5, (i)).

Proof. This follows from the definitions of $\mathfrak{D}_{X_{(r+1)}\{1,2\}}$ and $\Pi_{X_{(r+1)} \underline{\{1,2\}}}$. 


\section{Lemma 4.12.}

(i) The diagrams

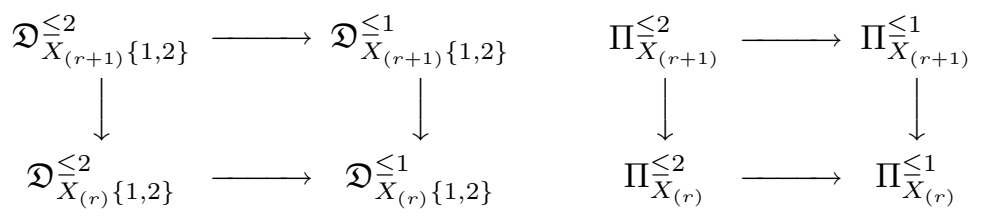

induced by the diagram

$$
\begin{aligned}
\Pi_{X_{(r+1)}}^{\mathrm{Lie} \leq 2} & \Pi_{X_{(r+1)}^{\mathrm{Lie}} \leq 1}^{\operatorname{via} p_{X_{(r)}: 3}^{\log } \downarrow} \\
\Pi_{X_{(r)}}^{\mathrm{Lie} \leq 2} & \downarrow{ }^{\operatorname{via} p_{X_{(r)}: 3}^{\log }} \longrightarrow \Pi_{X_{(r)}}^{\mathrm{Lie} \leq 1}
\end{aligned}
$$

are cartesian.

(ii) The subgroup of $\Pi_{X_{(r+1)}}^{\mathrm{Lie} \leq 2}$ obtained as the intersection of the inverse image of $\mathfrak{D}_{X_{(r+1)}\{1,2\}}^{\leq 1}$ (respectively, $\Pi_{X_{(r+1)}}^{\leq 1}$ ) via the natural projection

$$
\Pi_{X_{(r+1)}}^{\mathrm{Lie} \leq 2} \rightarrow \Pi_{X_{(r+1)}}^{\mathrm{Lie} \leq 1}
$$

and the inverse image of $\mathfrak{D}_{X_{(r)}}^{\leq 2}\{1,2\}$ (respectively, $\left.\Pi_{X_{(r)}}^{\leq 2}\right)$ via

$$
\Pi_{X_{(r+1)}}^{\mathrm{Lie} \leq 2} \stackrel{\text { via }}{p_{X_{(r)}: 3}^{\log }} \stackrel{\rightarrow}{\rightarrow} \Pi_{X_{(r)}}^{\mathrm{Lie} \leq 2}
$$

coincides with $\mathfrak{D}_{X_{(r+1)}}^{\leq 2}\{1,2\}$ (respectively, $\left.\Pi_{\bar{X}_{(r+1)}}^{\leq 2}\right)$.

Proof. Assertion (i) follows immediately from Lemma 4.5, (ii). Assertion (ii) follows from assertion (i), Lemma 4.13 below, together with the fact that the diagram

$$
\begin{aligned}
\Pi_{X_{(r+1)}}^{\mathrm{Lie} \leq 2} & \longrightarrow \Pi_{X_{(r+1)}}^{\mathrm{Lie} \leq 1} \\
\text { via }\left.p_{X_{(r)}: 3}^{\log }\right|_{\Pi_{X_{(r)}}^{\mathrm{Lie} \leq 2}} \longrightarrow \operatorname{via} p_{X_{(r)}: 3}^{\log } & \longrightarrow \Pi_{X_{(r)}}^{\mathrm{Lie} \leq 1}
\end{aligned}
$$

is cartesian (cf. Lemma 4.1, (ii)).

Lemma 4.13. Let

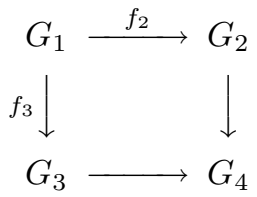


be a commutative diagram of groups, and $H_{1} \subseteq G_{1}$ a subgroup of $G_{1}$. Write $H_{2}$ (respectively, $\left.H_{3}\right)$ for the image of $H_{1}$ via $f_{2}$ (respectively, $f_{3}$ ). Then if the morphism

$$
H_{1} \longrightarrow H_{2} \times_{G_{4}} H_{3}
$$

induced by $f_{2}$ and $f_{3}$ is an isomorphism, and the intersection

$$
\operatorname{Ker} f_{2} \cap \operatorname{Ker} f_{3}
$$

is trivial (e.g., the above diagram is cartesian), then the natural inclusion morphism

$$
H_{1} \hookrightarrow f_{2}^{-1}\left(H_{2}\right) \cap f_{3}^{-1}\left(H_{3}\right)
$$

is an isomorphism.

Proof. Observe that the morphisms $f_{2}$ and $f_{3}$ induce a morphism

$$
f_{2}^{-1}\left(H_{2}\right) \cap f_{3}^{-1}\left(H_{3}\right) \longrightarrow H_{2} \times_{G_{4}} H_{3} .
$$

Since the composite

$$
H_{1} \hookrightarrow f_{2}^{-1}\left(H_{2}\right) \cap f_{3}^{-1}\left(H_{3}\right) \longrightarrow H_{2} \times_{G_{4}} H_{3}
$$

of the natural inclusion $H_{1} \hookrightarrow f_{2}^{-1}\left(H_{2}\right) \cap f_{3}^{-1}\left(H_{3}\right)$ and this morphism is an isomorphism by our assumption, we conclude that this morphism is surjective. Moreover, since $\operatorname{Ker} f_{2} \cap \operatorname{Ker} f_{3}$ is trivial, this morphism is an isomorphism. Then the assertion is immediate.

Lemma 4.14. The composite

$$
\mathfrak{D}_{X_{(r+1)}\{2,3\}}^{\leq 2} \hookrightarrow \Pi_{X_{(r+1)}}^{\leq 2} \stackrel{\text { via }}{p_{X(r)}^{\log }: 3} \Pi_{X_{(r)}}^{\leq 2}
$$

coincides with the composite

$$
\mathfrak{D}_{X_{(r+1)}\{2,3\}}^{\leq 2} \stackrel{\operatorname{via} p_{X_{(r)}}^{\log }: 3}{\rightarrow} \Pi_{X_{(r)}} \rightarrow \Pi_{X_{(r)}}^{\leq 2} .
$$

In particular, the morphism

$$
\mathfrak{D}_{X_{(r+1)}\{2,3\}}^{\leq 2} \stackrel{\sim}{\longrightarrow} \mathfrak{D}_{X_{(r+1)}\{2,3\}}^{\leq 1} \longrightarrow \Pi_{X_{(r+1)}}^{\leq 1} \times_{\Pi_{X_{(r)}}^{\leq 1}} \Pi_{X_{(r)}}^{\leq 2} \stackrel{\sim}{\sim} \Pi_{X_{(r+1)}}^{\leq 2}
$$

(cf. Lemmas 4.5, (ii); 4.12, (i)) determined by the natural inclusion $\mathfrak{D}_{X_{(r+1)}}^{\leq 1}\{2,3\}$ $\hookrightarrow \Pi_{X_{(r+1)}}^{\leq 1}$ and the composite $\mathfrak{D}_{X_{(r+1)}}^{\leq 1}\left\{\stackrel{\left.\text { via } p_{X_{(r)}}^{\log }: 3\right\}}{\rightarrow} \Pi_{X_{(r)}} \rightarrow \Pi_{X_{(r)}}^{\leq 2}\right.$ coincides with the natural inclusion $\mathfrak{D}_{X_{(r+1)}}^{\leq 2}\{2,3\} \hookrightarrow \Pi_{X_{(r+1)}}^{\leq 2}$. 
Proof. This follows immediately from Lemma 4.5, (ii).

Lemma 4.15 (cf. [16], Proposition 3.4, (ii)). $\quad$ Let $1 \leq i<j \leq r+1$ and $b \geq 2$ be integers. Then any two liftings of the natural inclusion $\mathfrak{D}_{X_{(r+1)}\{i, j\}}^{\leq b} \hookrightarrow$

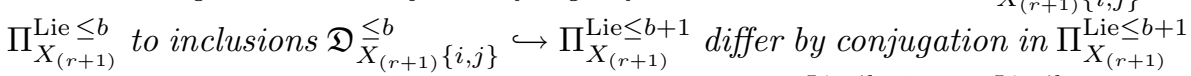
by a unique element of the kernel of the surjection $\Pi_{X_{(r+1)}^{\mathrm{Lie}} \leq b+1}^{\mathrm{L}} \rightarrow \Pi_{X_{(r+1)}}^{\mathrm{Lie} \leq b}$.

Proof. By Lemma 4.5, (ii), it is enough to show that

$$
H^{i}\left(\mathfrak{D}_{X_{(r+1)}}^{\leq b}\{i, j\}, \operatorname{Lin}_{X_{(r)}}^{\text {iner }}(b+1 / b+2)\right)=0
$$

for $i=0,1$. Since the action of $\mathfrak{D}_{X_{(r+1)}^{\Delta}\{i, j\}}^{\Delta}$ on $\operatorname{Lin}_{X_{(r)}}^{\text {iner }}(b+1 / b+2)$ is trivial, it thus suffices to observe (by considering the Hochschild-Serre spectral sequence associated to the surjection $\mathfrak{D}_{X_{(r+1)}\{i, j\}}^{\leq b} \rightarrow G_{K}$ ) that the action of $\operatorname{Fr}_{K}$ on $\operatorname{Lin}_{X_{(r)}}^{\text {iner }}(b+1 / b+2)$ is "of weight $b+1 \geq 3$ ", while the action of $\operatorname{Fr}_{K}$ on $\left(\mathfrak{D}_{X_{(r+1)}^{\Delta} \leq b}^{\Delta, j\}}\right)^{\mathrm{ab}}$ is "of weight $\leq 2$ " (cf. Proposition 4.2, (i)). This completes the proof of the assertion.

Lemma 4.16. Let

$$
\alpha_{(r)}: \Pi_{X_{(r)}} \stackrel{\sim}{\longrightarrow} \Pi_{Y_{(r)}}
$$

be a Frobenius-preserving and order-preserving isomorphism which is either tripod-preserving or the following condition $(*)$ holds:

(*): There exist

(i) a set $S=\left\{x_{1}, \cdots, x_{r}\right\}$ (respectively, $T \stackrel{\text { def }}{=}\left\{y_{1}, \cdots, y_{r}\right\}$ ) of distinct $K$-rational (respectively, L-rational) points of $X$ (respectively, $Y$ ) of cardinality $r$ with an ordering such that if a section $s_{S}: G_{K} \rightarrow$ $\Pi_{X_{(r)}}$ of the natural morphism $\Pi_{X_{(r)}} \rightarrow G_{K}$ corresponds to the $K$-rational point $\left(x_{1}, \cdots, x_{r}\right) \in U_{X_{(r)}}(K)$, then the section $t_{T}$ : $G_{L} \rightarrow \Pi_{Y_{(r)}}$ of the natural morphism $\Pi_{Y_{(r)}} \rightarrow G_{L}$ corresponding to $s_{S}$ (under the isomorphism $\alpha_{(r)}$ ) coincides with the section arising from the L-rational point $\left(y_{1}, \cdots, y_{r}\right) \in U_{Y_{(r)}}(L)(c f$. Proposition 2.5, (ii)), and

(ii) an isomorphism $\alpha_{S, T}: \Pi_{U_{S}} \stackrel{\sim}{\rightarrow} \Pi_{V_{T}}$ of the geometrically pro-l fundamental group $\Pi_{U_{S}}$ of $U_{S} \stackrel{\text { def }}{=} X \backslash S$ with the geometrically pro-l fundamental group $\Pi_{V_{T}}$ of $V_{T} \stackrel{\text { def }}{=} Y \backslash T$ such that the isomorphism $\Pi_{X} \stackrel{\sim}{\rightarrow} \Pi_{Y}$ induced by $\alpha_{S, T}$ coincides with the isomorphism $\alpha_{(1)}$ : $\Pi_{X} \stackrel{\sim}{\rightarrow} \Pi_{Y}$ induced by $\alpha_{(r)}$. 
Then there exists a unique isomorphism

$$
\alpha_{(r+1)}: \Pi_{X_{(r+1)}} \stackrel{\sim}{\longrightarrow} \Pi_{Y_{(r+1)}}
$$

well-defined up to composition with a $\operatorname{Ker}\left(\Pi_{Y_{(r+1)}} \rightarrow \Pi_{Y_{(r+1)}\{1,2\}}\right)$-inner automorphism such that, for $i=1, \cdots, r+1$, the following diagram commutes:

$$
\begin{aligned}
& \Pi_{X_{(r+1)}} \stackrel{\alpha_{(r+1)}}{\longrightarrow} \Pi_{Y_{(r+1)}} \quad \downarrow \text { via } p_{U_{Y_{(r)}}: i} \\
& \text { via } p_{U_{X_{(r)}}: i} \downarrow \\
& \Pi_{X_{(r)}} \stackrel{\alpha_{(r)}}{\longrightarrow} \Pi_{Y_{(r)}} .
\end{aligned}
$$

Proof. If $\alpha_{(r)}$ is tripod-preserving (respectively, satisfies the condition $(*)$ ), then we assume that the section $s_{0}$ satisfies the condition $\left(\dagger_{\mathbb{P}}\right)$ (respectively, $\left.\left(\dagger_{S}\right)\right)$. Then since $\alpha_{(r)}$ is Frobenius-preserving, it follows immediately from the naturality of our construction that $\alpha_{(r)}$ induces, for each positive integer $b$, isomorphisms

$$
\alpha_{(r+1)}^{\mathrm{Lie} \leq b}: \Pi_{X_{(r+1)}}^{\mathrm{Lie} \leq b} \stackrel{\sim}{\longrightarrow} \Pi_{Y_{(r+1)}}^{\mathrm{Lie} \leq b} ; \alpha_{(r)}^{\mathrm{Lie} \leq b}: \Pi_{X_{(r)}}^{\mathrm{Lie} \leq b} \stackrel{\sim}{\longrightarrow} \Pi_{Y_{(r)}}^{\mathrm{Lie} \leq b}
$$

that fit into the following commutative diagrams:

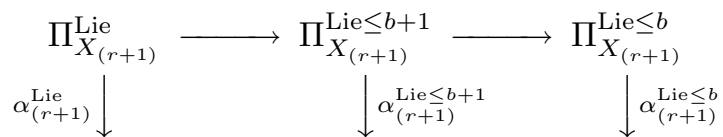

$$
\begin{aligned}
& \Pi_{Y_{(r+1)}}^{\mathrm{Lie}} \longrightarrow \Pi_{Y_{(r+1)}}^{\mathrm{Lie} \leq b+1} \longrightarrow \Pi_{Y_{(r+1)}^{\mathrm{Lie}} \leq b}^{\mathrm{L}}
\end{aligned}
$$

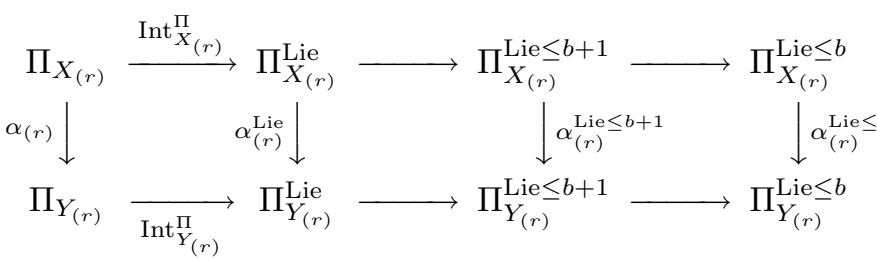

$$
\begin{aligned}
& \Pi_{X_{(r+1)}}^{\mathrm{Lie} \leq b} \stackrel{\text { via } p_{U_{X_{(r)}}: 3}}{\longrightarrow} \Pi_{X_{(r)}}^{\mathrm{Lie} \leq b} \\
& \alpha_{(r+1)}^{\mathrm{Lie} \leq b} \downarrow \downarrow \downarrow \alpha_{(r)}^{\mathrm{Lie} \leq b} \\
& \Pi_{Y_{(r+1)}}^{\mathrm{Lie} \leq b} \underset{\text { via } p_{U_{Y_{(r)}}: 3}}{\longrightarrow} \Pi_{Y_{(r)}}^{\mathrm{Lie} \leq b}
\end{aligned}
$$

(cf. Lemma 4.7).

Moreover, since $\alpha_{(r+1)}^{\mathrm{Lie} \leq b}$ is compatible with the Frobenius elements on either side, (by the assumption on the section $s_{0}$ fixed in the discussion preceding 
Proposition 4.2) $\alpha_{(r+1)}^{\mathrm{Lie} \leq b}$ maps $\mathfrak{I}_{X_{(r+1)}\{1,2\}}^{\leq b}$ bijectively onto $\mathfrak{I}_{Y_{(r+1)}}^{\leq b}\{1,2\}$. In particular, $\alpha_{(r+1)}^{\mathrm{Lie} \leq b}$ maps

$$
\mathfrak{I}_{X_{(r+1)}\{1,2\}}^{\leq b} \rtimes G_{K}\left(\subseteq \Pi_{X_{(r+1)}}^{\mathrm{Lie} \leq b}\right)
$$

bijectively onto

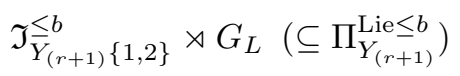

[where we note that, by the assumption on the section $s_{0}, \mathfrak{I}_{X_{(r+1)}\{1,2\}}^{\leq b}$ (respectively, $\mathfrak{I}_{\bar{Y}_{(r+1)}^{\leq b}\{1,2\}}^{\leq b}$ ) is stable under the action of $G_{K}$ (respectively, $G_{L}$ ) on $\Pi_{X_{(r+1)}}^{\mathrm{Lie} \leq b} \quad\left(\right.$ respectively, $\left.\left.\Pi_{Y_{(r+1)}}^{\mathrm{Lie} \leq b}\right)\right]$.

On the other hand, if $\alpha_{(r+1)}$ is tripod-preserving (respectively, satisfies the condition $(*)$ ), then it follows from Lemma 4.8 (respectively, Lemma 4.9),

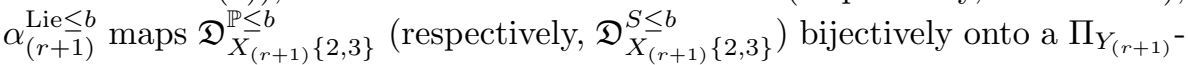
conjugate of $\mathfrak{D}_{Y_{(r+1)} \mathbb{P} \leq b}^{\mathbb{P}}\{2,3\}$ (respectively, $\mathfrak{D}_{Y_{(r+1)}^{T \leq b}\{2,3\}}^{T}$ ) [cf. the notation of Lemma 4.8 (respectively, Lemma 4.9$)$ ], where for " $(-) "=\mathbb{P}, S$, or $T$, and " $\left(-^{\prime}\right) "=X$ or $Y, \mathfrak{D}_{\left(-^{\prime}\right)_{(r+1)}^{(-)}\{2,3\}}^{(\text {is }}$ the image of the composite

$$
\mathfrak{D}_{\left(--^{\prime}\right)_{(r+1)}\{2,3\}}^{(-)} \hookrightarrow \Pi_{\left(-^{\prime}\right)_{(r+1)}} \stackrel{\operatorname{Int}_{\left(-^{\prime}\right)}^{\Pi}(r+1)}{\longrightarrow} \Pi_{\left(-^{\prime}\right)_{(r+1)}}^{\mathrm{Lie}} \rightarrow \Pi_{\left(-^{\prime}\right)_{(r+1)}}^{\mathrm{Lie} \leq b} .
$$

First, I claim that the isomorphism $\alpha_{(r+1)}^{\mathrm{Lie} \leq 1}$ of $\Pi_{X_{(r+1)} \underline{\{1,2\}}}$ with $\Pi_{Y_{(r+1)} \underline{\{1,2\}}}$ (cf. Lemma 4.5, (i)) induces a bijection between the set of

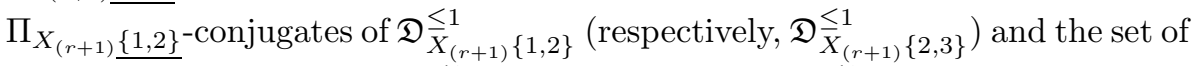

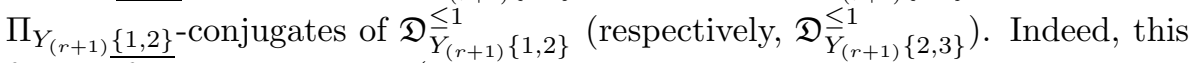
follows from Lemma 4.11 (respectively, a similar argument to the argument used in the proof of Proposition 2.5, (iii)).

Next, I claim that the isomorphism $\alpha_{(r+1)}^{\mathrm{Lie} \leq 2}$ induces a bijection between the set of $\Pi_{X_{(r+1)}}^{\leq 2}$-conjugates of $\mathfrak{D}_{X_{(r+1)}}^{\leq 2}\{1,2\}$ (respectively, $\left.\mathfrak{D}_{X_{(r+1)}}^{\leq 2}\{2,3\}\right)$ and the set of $\Pi_{Y_{(r+1)}}^{\leq 2}$-conjugates of $\mathfrak{D}_{Y_{(r+1)}}^{\leq 2}\{1,2\}$ (respectively, $\mathfrak{D}_{Y_{(r+1)}}^{\leq 2}\{2,3\}$ ). Indeed, this follows from the claim just verified above, together with Lemma 4.12, (ii) (respectively, Lemma 4.12, (ii), together with Lemma 4.14).

Next, I claim that the isomorphism $\alpha_{(r+1)}^{\mathrm{Lie} \leq b}$ induces a bijection between the set of $\Pi_{X_{(r+1)}}^{\leq b}$-conjugates of $\mathfrak{D}_{X_{(r+1)}\{1,2\}}^{\leq b}$ (respectively, $\mathfrak{D}_{X_{(r+1)} \leq b}^{\leq b, 3\}}$ ) and the set of $\Pi_{Y_{(r+1)}}^{\leq b}$-conjugates of $\mathfrak{D}_{Y_{(r+1)} \leq b}^{\leq b}\left\{\right.$ (respectively, $\mathfrak{D}_{Y_{(r+1)}}^{\leq b}\{2,3\}$ ) for each positive integer $b$. To verify this claim, we apply induction on $b$. The case where $b=1$ or 2 is verified above. Thus, we assume that $b \geq 2$, and that the claim has been verified for " $b$ " that are $\leq$ the $b$ under consideration. Now 
observe that it follows from Lemma 4.15 that any two liftings of the natural inclusion $\mathfrak{D}_{X_{(r+1)}\{1,2\}}^{\leq b} \hookrightarrow \Pi_{X_{(r+1)}}^{\mathrm{Lie} \leq b}$ (respectively, $\mathfrak{D}_{X_{(r+1)}\{2,3\}}^{\leq b} \hookrightarrow \Pi_{X_{(r+1)}}^{\mathrm{Lie} \leq b}$ ) to inclusions $\mathfrak{D}_{X_{(r+1)} \leq b}^{\leq 1,2\}} \hookrightarrow \Pi_{X_{(r+1)}^{\mathrm{Lie} \leq b+1}}^{\mathrm{L}}$ (respectively, $\mathfrak{D}_{X_{(r+1)}\{b}^{\leq b\}} \hookrightarrow \Pi_{X_{(r+1)}^{\mathrm{Lie}} \leq b+1}^{\mathrm{L}}$ ) differ by conjugation in $\Pi_{X_{(r+1)}}^{\mathrm{Lie} \leq b+1}$ by a unique element of the kernel of the surjection $\Pi_{X_{(r+1)}}^{\mathrm{Lie} \leq b+1} \rightarrow \Pi_{X_{(r+1)}}^{\mathrm{Lie} \leq b}$; moreover, it follows from the definition that the kernel of the surjection $\Pi_{X_{(r+1)}^{\mathrm{Lie} \leq b+1}}^{\mathrm{L}} \rightarrow \Pi_{X_{(r+1)}}^{\mathrm{Lie} \leq b}$ is included in the center of $\Delta_{X_{(r+1)}}^{\text {Lie } \leq b+1}$. Therefore, the restriction to $\mathfrak{D}_{X_{(r+1)}^{\Delta \leq b}\{1,2\}}^{\Delta}$ (respectively, $\left.\mathfrak{D}_{\left.X_{(r+1)} \leq b, 3\right\}}^{\Delta \leq b}\right)$ of any lifting of the natural inclusion $\mathfrak{D}_{X_{(r+1)}\{1,2\}}^{\leq b} \hookrightarrow \Pi_{X_{(r+1)}}^{\text {Lie } \leq b}$ (respectively, $\mathfrak{D}_{X_{(r+1)}\{b, 3\}}^{\leq} \hookrightarrow \Pi_{X_{(r+1)}}^{\text {Lie } \leq b}$ ) to an inclusion $\mathfrak{D}_{X_{(r+1)}\{1,2\}}^{\leq b} \Pi_{X_{(r+1)}}^{\text {Lie } \leq b+1}$ (respectively, $\left.\mathfrak{D}_{X_{(r+1)}\{2,3\}}^{\leq b} \hookrightarrow \Pi_{X_{(r+1)}}^{\mathrm{Lie} \leq b+1}\right)$ coincides with the natural inclusion $\mathfrak{D}_{X_{(r+1)}^{\Delta}\{b+1,2\}}^{\Delta} \hookrightarrow$ $\Pi_{X_{(r+1)}}^{\mathrm{Lie} \leq b+1}$ (respectively, $\left.\mathfrak{D}_{X_{(r+1)}^{\Delta} \leq b+1}^{\Delta} \hookrightarrow \Pi_{X_{(r+1)}}^{\mathrm{Lie} \leq b+1}\right)$. Thus, it follows that the isomorphism $\alpha_{(r+1)}^{\mathrm{Lie} \leq b+1}$ induces a bijection between the set of $\Delta_{\bar{X}_{(r+1)}}^{\leq b+1}$-conjugates of $\mathfrak{D}_{\left.X_{(r+1)} \leq b+1,2\right\}}^{\Delta \leq b+1}$ (respectively, $\mathfrak{D}_{X_{(r+1)} \leq b+1}^{\Delta}\{2,3\}$ ) and the set of $\Delta_{Y_{(r+1)}}^{\leq b+1}$-conjugates of $\mathfrak{D}_{Y_{(r+1)}^{\Delta \leq b+1,2\}}}^{\Delta \leq b+1}$ (respectively, $\left.\mathfrak{D}_{Y_{(r+1)}^{\Delta \leq b+1}\{2\}}^{\Delta}\right)$; in particular, since $\alpha_{(r+1)}^{\mathrm{Lie} \leq b+1}$ is compatible with the Frobenius elements on either side, it follows from Lemma 4.4 that the isomorphism $\alpha_{(r+1)}^{\mathrm{Lie} \leq b+1}$ maps $\Pi_{X_{(r+1)}}^{\leq b+1}$ bijectively onto $\Pi_{Y_{(r+1)}}^{\leq b+1}$. Moreover, observe that the subgroup $\mathfrak{D}_{X_{(r+1)}}^{\leq b+1}\{1,2\} \subseteq \Pi_{X_{(r+1)}}^{\mathrm{Lie} \leq b}$ is a subgroup which is uniquely determined by the following condition that it be a

the image of a lifting of the natural inclusion $\mathfrak{D}_{X_{(r+1)}\{1,2\}}^{\leq b} \hookrightarrow \Pi_{X_{(r+1)}}^{\mathrm{Lie} \leq b}$

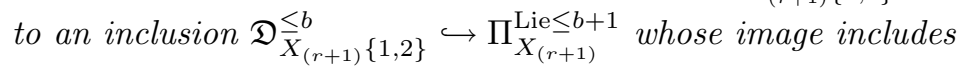

$$
\mathfrak{I}_{X_{(r+1)}\{1,2\}}^{\leq b+1} \rtimes G_{K} .
$$

(Indeed, the assertion that this condition uniquely determines the subgroup $\mathfrak{D}_{X_{(r+1)} \leq b+1}^{{ }_{1,2\}}} \subseteq \Pi_{X_{(r+1)}}^{\mathrm{Lie} \leq b+1}$ may be verified follows: First, let us observe that the isomorphism $\mathfrak{D}_{X_{(r+1)}\{1,2\}}^{\leq b+1} \stackrel{\sim}{\rightarrow} \mathfrak{D}_{X_{(r+1)}\{1,2\}}^{\leq b}$ induced by the natural projection $\Pi_{X_{(r+1)}}^{\mathrm{Lie} \leq b+1} \rightarrow \Pi_{X_{(r+1)}}^{\mathrm{Lie} \leq b}\left[\right.$ cf. Lemma 4.5 , (ii)] induces an isomorphism $\mathfrak{I}_{X_{(r+1)}\{b+1,2\}}^{\leq b} \rtimes$ $G_{K} \stackrel{\sim}{\rightarrow} \mathfrak{I}_{X_{(r+1)}}^{\leq b 1,2\}} \underset{ }{ } \rtimes G_{K}$. Thus, any two liftings of the natural inclusion $\mathfrak{D}_{X_{(r)}\{1,2\}}^{\leq b} \hookrightarrow \Pi_{X_{(r+1)}}^{\mathrm{Lie} \leq b}$ to inclusions $\mathfrak{D}_{X_{(r)}\{1,2\}}^{\leq b} \hookrightarrow \Pi_{X_{(r+1)}}^{\mathrm{Lie} \leq b+1}$ whose images include

$$
\Im_{X_{(r+1)}\{b+1,2\}}^{\leq b} \rtimes G_{K} \subseteq \Pi_{X_{(r+1)}^{\mathrm{Lie} \leq b+1}}^{\mathrm{Lin}}
$$

[since $b \geq 2]$ in fact coincide on $\mathfrak{I}_{X_{(r+1)}\{1,2\}}^{\leq b} \rtimes G_{K} \subseteq \Pi_{X_{(r+1)}}^{\text {Lie } \leq b}$. Therefore, by Lemma 4.15, it is enough to verify that the submodule of $\operatorname{Fr}_{K}$-invariants of 


$$
\operatorname{Ker}\left(\Pi_{X_{(r+1)}^{\mathrm{Lie}} \leq b+1}^{\mathrm{L}} \rightarrow \Pi_{X_{(r+1)}}^{\mathrm{Lie} \leq b}\right)=\operatorname{Lin}_{X_{(r+1)}}^{\text {iner }}(b+1 / b+2)
$$

[cf. Lemma 4.5, (ii)] is zero. However, this follows immediately from Proposition 4.2 , (i).) Now by considering a similar condition for $\mathfrak{D}_{Y_{(r+1)}\{1,2\}}^{\leq b} \subseteq$ $\Pi_{Y_{(r+1)}}^{\mathrm{Lie} \leq b+1}$, the claim that the isomorphism $\alpha_{(r+1)}^{\mathrm{Lie} \leq b+1}$ induces a bijection between the set of $\Pi_{X}^{\leq b+1}$-conjugates of $\mathfrak{D}_{X_{(r+1)}}^{\leq b+1}\{1,2\}$ and the set of $\Pi_{\bar{Y}_{(r+1)}}^{\leq b+1}$ conjugates of $\mathfrak{D}_{Y_{(r+1)}\{b+1,2\}}^{\leq b o l l o w s}$ from the fact that the isomorphism $\alpha_{(r+1)}^{\mathrm{Lie} \leq b+1}$ maps $\mathfrak{I}_{X_{(r+1)}^{\leq b+1,2\}}}^{\leq b} \rtimes G_{K}$ bijectively onto $\mathfrak{I}_{Y_{(r+1)}}^{\leq b+1,2\}}{ }_{(1,1} G_{L}$, together with the fact that the isomorphism $\alpha_{(r+1)}^{\mathrm{Lie} \leq b+1}$ maps $\Pi_{X_{(r+1)}}^{\leq b+1}$ bijectively onto $\Pi_{Y_{(r+1)}}^{\leq b+1}$. On the other hand, by replacing $\{1,2\}$ by $\{2,3\}, \mathfrak{I}_{X_{(r+1)}\{1,2\}}^{\leq b+1} \rtimes G_{K}$ by

$$
\left\{\begin{array}{l}
\mathfrak{D}_{\left.X_{(r+1)} \leq b+3\right\}}^{\mathbb{P} \leq b+1}\left(\text { if } \alpha_{(r)}\right. \text { is tripod-preserving) } \\
\mathfrak{D}_{\left.X_{(r+1)} \leq b+3\right\}}\left(\text { if } \alpha_{(r)} \text { satisfies }(*)\right)
\end{array}\right.
$$

and $\mathfrak{I}_{Y_{(r+1)}}^{\leq b+1,2\}} \rtimes G_{L}$ by

$$
\left\{\begin{array}{l}
\mathfrak{D}_{Y_{(r+1)}^{\mathbb{P} \leq b+1}\{3\}}^{\mathbb{P} \leq b+1} \\
\mathfrak{D}_{Y_{(r+1)}^{T}\{2,3\}}^{T}\left(\text { if } \alpha_{(r)}\right. \text { is tripod-preserving) } \\
\text { satisfies }(*))
\end{array}\right.
$$

it follows from a similar argument to the argument used in the proof of the assertion that the isomorphism $\alpha_{(r+1)}^{\mathrm{Lie} \leq b+1}$ induces a bijection between the set of $\Pi_{X}^{\leq b+1}$-conjugates of $\mathfrak{D}_{X_{(r+1)}}^{\leq b+1}\{1,2\}$ and the set of $\Pi_{Y_{(r+1)}}^{\leq b+1}$-conjugates of $\mathfrak{D}_{Y_{(r+1)}^{\leq b+1}\{1,2\}}^{\leq b}$ that the isomorphism $\alpha_{(r+1)}^{\mathrm{Lie} \leq b+1}$ induces a bijection between the set of $\Pi_{\bar{X}_{(r+1)}}^{\leq b+1}$-conjugates of $\mathfrak{D}_{X_{(r+1)}\{2,3\}}^{\leq b+1}$ and the set of $\Pi_{\bar{Y}_{(r+1)}}^{\leq b+1}$-conjugates of $\mathfrak{D}_{Y_{(r+1)}^{\leq b+1}}^{\leq b 2,3\}} \cdot$

By the various claims verified above, by taking the projective limit, we thus conclude that the isomorphism $\alpha_{(r+1)}^{\text {Lie }}$ induces an isomorphism of $\Pi_{X_{(r+1)}}$ with $\Pi_{Y_{(r+1)}}$ by Lemma 4.4 .

Finally, we note that the indeterminacy, referred to in the statement of Lemma 4.16, of the isomorphism $\alpha_{(r+1)}$ up to composition with a cuspidally inner automorphism arises precisely from the indeterminacy of the choice of the subgroups $\mathfrak{I}_{X_{(r+1)}\{i, j\}} \subseteq \Pi_{X_{(r+1)}}, \mathfrak{I}_{Y_{(r+1)}\left\{i^{\prime}, j^{\prime}\right\}} \subseteq \Pi_{Y_{(r+1)}}(1 \leq i<j \leq r+1,1 \leq$ $\left.i^{\prime}<j^{\prime} \leq r+1\right)$ and the sections of the natural morphisms $\Pi_{X_{(r+1)}} \rightarrow G_{K}$ and $\Pi_{Y_{(r+1)}} \rightarrow G_{L}$ (cf. Remark 8) with respect to cuspidally inner automorphisms of $\Pi_{X_{(r+1)}}, \Pi_{Y_{(r+1)}}$, respectively.

Lemma 4.17. Any Frobenius-preserving isomorphisms of $\Pi_{X}$ with $\Pi_{Y}$ are tripod-preserving. 
Proof. Let $\alpha$ be a Frobenius-preserving isomorphism of $\Pi_{X}$ with $\Pi_{Y}$. Note that since replacing the base field by a finite extension field of the base field does not affect the validity of the assertion that $\alpha$ is tripod-preserving, we may assume that there exists a $K$-rational point $x$ of $X$, and a non-trivial finite étale Galois covering which is geometrically pro-l and completely split above $x$.

Then it follows from [16], Theorem 3.1, that there exists an isomorphism $\alpha_{(2)}$ of $\Pi_{X_{(2)}}$ with $\Pi_{Y_{(2)}}$ which fits into a commutative diagram

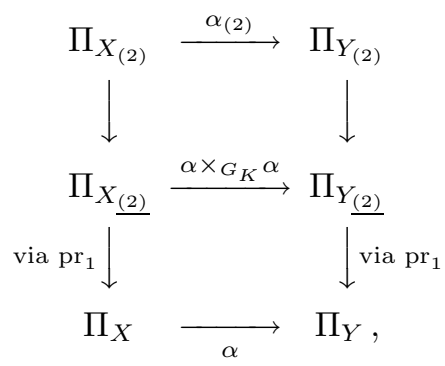

where the left-hand (respectively, right-hand) top vertical arrow is the morphism induced by the natural open immersion $U_{X_{(2)}} \hookrightarrow X \times_{K} X$ (respectively, $\left.U_{Y_{(2)}} \hookrightarrow Y \times_{L} Y\right)$. By base-changing the above diagram via the section $G_{K} \rightarrow \Pi_{X}$ arising from the $K$-rational point $x$ of $X$ and the section of the natural morphism $\Pi_{Y} \rightarrow G_{L}$ corresponding to the section $G_{K} \rightarrow \Pi_{X}$ (under the isomorphism $\alpha$ ), we obtain a commutative diagram

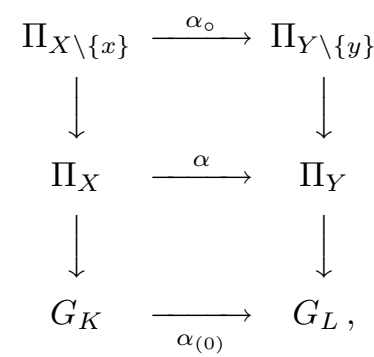

where $y$ is an $L$-rational point of $Y$ such that the section arising from $y$ coincides with the section of the natural morphism $\Pi_{Y} \rightarrow G_{L}$ corresponding to the section $G_{K} \rightarrow \Pi_{X}$ arising from the $K$-rational point $x$ of $X$ (cf. Proposition 2.5, (ii)). Let $X^{\prime} \rightarrow X$ be a non-trivial Galois covering of $X$ which is geometrically pro-l and completely split above $x$, and $Y^{\prime} \rightarrow Y$ the Galois covering over $Y$ corresponding to $X^{\prime} \rightarrow X$ (under the isomorphism $\alpha$ ). Then by base-changing the above diagram via the natural inclusions $\Pi_{X^{\prime}} \hookrightarrow \Pi_{X}$ and $\Pi_{Y^{\prime}} \hookrightarrow \Pi_{Y}$, we 
obtain a commutative diagram

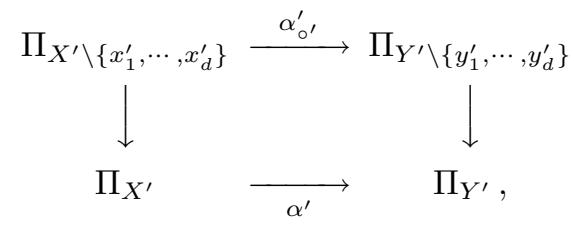

where $d$ is the degree of the covering $X^{\prime} \rightarrow X$, and $\left\{x_{1}^{\prime}, \cdots, x_{d}^{\prime}\right\}$ (respectively, $\left\{y_{1}^{\prime}, \cdots, y_{d}^{\prime}\right\}$ ) is the subset of $X^{\prime}(K)$ (respectively, $Y^{\prime}(L)$ ) obtained as the inverse image of $\{x\}$ (respectively, $\{y\}$ ) via the morphism $X^{\prime}(K) \rightarrow X(K)$ (respectively, $Y^{\prime}(L) \rightarrow Y(L)$ ); in particular, we obtain a commutative diagram:

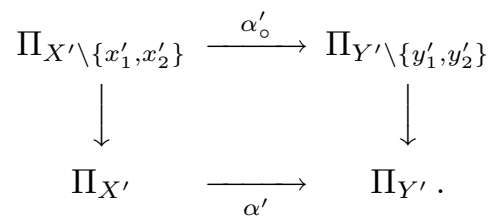

Now we assume that the decomposition subgroup $\mathfrak{D}_{x_{i}^{\prime}} \subseteq \Pi_{X^{\prime} \backslash\left\{x_{1}^{\prime}, x_{2}^{\prime}\right\}}$ associated to $x_{i}^{\prime}$ (well-defined, up to conjugate) corresponds to the decomposition subgroup $\mathfrak{D}_{y_{i}^{\prime}} \subseteq \Pi_{Y^{\prime} \backslash\left\{y_{1}^{\prime}, y_{2}^{\prime}\right\}}$ associated to $y_{i}^{\prime}$ (well-defined, up to conjugate) under the isomorphism $\alpha_{\circ}^{\prime}$.

Now I claim that the section of the natural morphism $\Pi_{X_{(2)}^{\prime}} \rightarrow G_{K}$ arising from $\left(x_{1}^{\prime}, x_{2}^{\prime}\right) \in U_{X^{\prime}(2)}(K)$ corresponds to the section of the natural morphism $\Pi_{Y_{(2)}^{\prime}} \rightarrow G_{L}$ arising from $\left(y_{1}^{\prime}, y_{2}^{\prime}\right) \in U_{Y_{(2)}^{\prime}}(L)$ under the isomorphism $\alpha_{(2)}^{\prime}$ of $\Pi_{X_{(2)}^{\prime}}$ with $\Pi_{Y_{(2)}^{\prime}}$ obtained from $\alpha^{\prime}$ (cf. [16], Theorem 3.1). Indeed, it follows from Proposition 4.6 that we may assume that the top horizontal arrow in the diagram

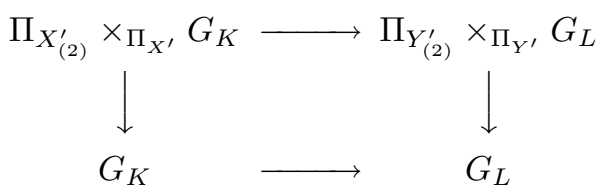

obtained by base-changing the diagram

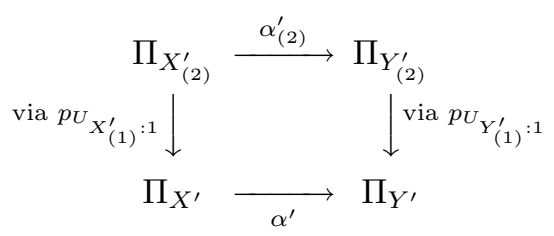

via the morphism $G_{K} \rightarrow \Pi_{X^{\prime}}$ induced by the composite $\mathfrak{D}_{x_{1}^{\prime}} \hookrightarrow \Pi_{X^{\prime} \backslash\left\{x_{1}^{\prime}, x_{2}^{\prime}\right\}} \rightarrow$ $\Pi_{X^{\prime}}$ and the morphism $G_{L} \rightarrow \Pi_{Y^{\prime}}$ induced by the composite $\mathfrak{D}_{y_{1}^{\prime}} \hookrightarrow \Pi_{Y^{\prime}} \backslash\left\{y_{1}^{\prime}, y_{2}^{\prime}\right\}$ 
$\rightarrow \Pi_{Y^{\prime}}$ coincides with the isomorphism of $\Pi_{X^{\prime} \backslash\left\{x_{1}^{\prime}\right\}}$ with $\Pi_{Y^{\prime} \backslash\left\{y_{1}^{\prime}\right\}}$ induced by $\alpha_{\circ}^{\prime}$. Thus, the claim follows from the fact that the composite $\mathfrak{D}_{x_{2}^{\prime}} \hookrightarrow$

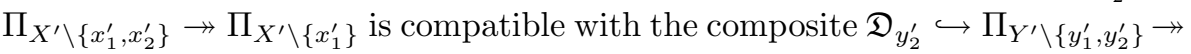
$\Pi_{Y^{\prime} \backslash\left\{y_{1}^{\prime}\right\}}$ under the isomorphism of $\Pi_{X^{\prime} \backslash\left\{x_{1}^{\prime}\right\}}$ with $\Pi_{Y^{\prime}} \backslash\left\{y_{1}^{\prime}\right\}$ induced by $\alpha_{\circ}^{\prime}$.

By the above claim just verified, the isomorphism $\alpha_{(2)}^{\prime}$ satisfies the condition $(*)$ in the statement of Lemma 4.16 ; in particular, it follows from Lemma 4.16 that $\alpha_{(2)}^{\prime}$ extends to an isomorphism of $\Pi_{X_{(3)}^{\prime}}$ with $\Pi_{Y_{(3)}^{\prime}}$. Thus, it follows from Lemma 3.1 that $\alpha^{\prime}$, hence also $\alpha$ is tripod-preserving (cf. [16], Remark 1).

The main result of this paper is as follows:

Theorem 4.1. $\quad$ Let $X($ respectively, $Y)$ be a proper hyperbolic curve over a finite field $K$ (respectively, L). Let

$$
\alpha_{(1)}: \Pi_{X} \stackrel{\sim}{\longrightarrow} \Pi_{Y}
$$

be a Frobenius-preserving isomorphism. Then, for any positive integer $r$, there exists a unique isomorphism

$$
\alpha_{(r)}: \Pi_{X_{(r)}} \stackrel{\sim}{\longrightarrow} \Pi_{Y_{(r)}},
$$

well-defined up to composition with a cuspidally inner automorphism (i.e., a $\operatorname{Ker}\left(\Pi_{Y_{(r)}} \rightarrow \Pi_{Y_{(r)}}\right)$-inner automorphism), which is compatible with the natural respective actions of the symmetric group on $r$ letters such that, for $i=$ $1, \cdots, r+1$, the following diagram commutes up to composition with a cuspidally inner automorphism (i.e., a $\operatorname{Ker}\left(\Pi_{Y_{(r)}} \rightarrow \Pi_{Y_{\underline{(r)}}}\right)$-inner automorphism):

$$
\begin{aligned}
\Pi_{X_{(r+1)}} \stackrel{\alpha_{(r+1)}}{\longrightarrow} \Pi_{Y_{(r+1)}} & \\
\text { via } p_{U_{X_{(r)}}: i} \downarrow & \\
\Pi_{X_{(r)}} & \stackrel{\downarrow}{\alpha_{(r)}} \Pi_{Y_{(r)}} .
\end{aligned}
$$

Proof. The assertion in the case where $r=2$ follows from [16], Theorem 3.1; on the other hand, the assertion in the case where $r \geq 3$ follows from induction on $r$, together with Proposition 4.6; Lemmas 4.16; 4.17.

The following corollary follows immediately from Theorem 4.1, together with the fact that a hyperbolic curve over a finite field is $\mathfrak{P r i m e}$-separated (cf. [16], Definition 1.5, (i); Proposition 2.2, (ii)). 
Corollary 4.1. $\quad$ Let $X$ (respectively, $Y$ ) be a proper hyperbolic curve over a finite field $K$ (respectively, $L)$.

(i) Let

$$
\alpha: \Pi_{X} \stackrel{\sim}{\longrightarrow} \Pi_{Y}
$$

be a Frobenius-preserving isomorphism of the geometrically pro-l fundamental group of $X$ with the geometrically pro-l fundamental group of $Y, r$ a positive integer, and $\left\{x_{1}, \cdots, x_{r}\right\}$ a set of distinct $K$-rational points of $X$ of cardinality $r$ with an ordering. Then there exists a set $\left\{y_{1}, \cdots, y_{r}\right\}$ of distinct L-rational points of $Y$ of cardinality $r$ with an ordering, and an isomorphism

$$
\alpha^{\text {new }}: \Pi_{X \backslash\left\{x_{1}, \cdots, x_{r}\right\}} \stackrel{\sim}{\longrightarrow} \Pi_{Y \backslash\left\{y_{1}, \cdots, y_{r}\right\}}
$$

of the geometrically pro-l fundamental group of $X \backslash\left\{x_{1}, \cdots, x_{r}\right\}$ with the geometrically pro-l fundamental group of $Y \backslash\left\{y_{1}, \cdots, y_{r}\right\}$ which is compatible with $\alpha$. Moreover, such an isomorphism $\alpha^{\text {new }}$ is uniquely determined up to composition with a cuspidally inner automorphism (i.e., a $\operatorname{Ker}\left(\Pi_{Y \backslash\left\{y_{1}, \cdots, y_{r}\right\}} \rightarrow \Pi_{Y}\right)$-inner automorphism $)$.

(ii) Let

$$
\alpha: \pi_{1}(X) \stackrel{\sim}{\longrightarrow} \pi_{1}(Y)
$$

be a Frobenius-preserving isomorphism of the (profinite) fundamental group of $X$ with the (profinite) fundamental group of $Y, r$ a positive integer, and $\left\{x_{1}, \cdots, x_{r}\right\}$ a set of distinct $K$-rational points of $X$ of cardinality $r$. Then there exists a set $\left\{y_{1}, \cdots, y_{r}\right\}$ of distinct L-rational points of $Y$ of cardinality $r$, and an isomorphism

$$
\alpha^{\mathrm{cp}(l)}: \pi_{1}\left(X \backslash\left\{x_{1}, \cdots, x_{r}\right\}\right)^{\operatorname{cp}(l)} \stackrel{\sim}{\longrightarrow} \pi_{1}\left(Y \backslash\left\{y_{1}, \cdots, y_{r}\right\}\right)^{\operatorname{cp}(l)}
$$

of the maximal cuspidally pro-l quotient (cf. [16], Definition 1.1, (i)) of $\pi_{1}\left(X \backslash\left\{x_{1}, \cdots, x_{r}\right\}\right)$ (relative to $\left.\pi_{1}\left(X \backslash\left\{x_{1}, \cdots, x_{r}\right\}\right) \rightarrow \pi_{1}(X)\right)$ with the maximal cuspidally pro-l quotient of $\pi_{1}\left(Y \backslash\left\{y_{1}, \cdots, y_{r}\right\}\right)$ (relative to $\left.\pi_{1}\left(Y \backslash\left\{y_{1}, \cdots, y_{r}\right\}\right) \rightarrow \pi_{1}(Y)\right)$ which is compatible with $\alpha$. Moreover, such an isomorphism $\alpha^{\mathrm{cp}(l)}$ is uniquely determined up to composition with a cuspidally inner automorphism (i.e., a $\operatorname{Ker}\left(\pi_{1}\left(Y \backslash\left\{y_{1}, \cdots, y_{r}\right\}\right)^{\operatorname{cp}(l)} \rightarrow \pi_{1}(Y)\right)$ inner automorphism).

Remark 9 .

(i) Since a hyperbolic curve over a finite field is not $l$-separated (cf. [16], Definition 1.5, (i)) in general (cf. Remark 10, (i) below), the " $y_{i}$ 's" (hence 
also " $\alpha$ new") in the statement of Corollary $4.1,(\mathrm{i})$, depend, unlike the case with Corollary 4.1, (ii), on the ordering of $\left\{x_{1}, \cdots, x_{r}\right\}$.

(ii) In the notation of Corollary 4.1, (ii), since it follows from [16], Theorem 3.2 , that there exists a unique isomorphism (of schemes) of $\phi: X \stackrel{\sim}{\rightarrow} Y$ such that the isomorphism $\pi_{1}(X) \stackrel{\sim}{\rightarrow} \pi_{1}(Y)$ induced on fundamental groups by $\phi$ coincides with $\alpha$, it follows immediately that there exists an isomorphism $\pi_{1}\left(X \backslash\left\{x_{1}, \cdots, x_{r}\right\}\right) \stackrel{\sim}{\rightarrow} \pi_{1}\left(Y \backslash\left\{y_{1}, \cdots, y_{r}\right\}\right)$ which is compatible with $\alpha$. On the other hand, Corollary 4.1, (ii), provides a direct way to construct such an isomorphism between the cuspidally pro-l fundamental groups, i.e., a way to construct such an isomorphism without passing through "the world of schemes".

Remark 10.

(i) In general, a hyperbolic curve over a finite field is not l-separated. The following example of this phenomenon was given by Akio Tamagawa:

Let $X$ be a proper hyperbolic curve over a finite field $K$ of characteristic $p$, and $\bar{K}$ an algebraic closure of $K$. Let us denote by $\Pi_{X}$ the geometrically pro- $l$ fundamental group of $X$ (where $l$ is a prime number such that $l \neq p$ ), by $\Delta_{X}$ the pro- $l$ fundamental group of $X \otimes_{K} \bar{K}$, and by $G_{K}$ the Galois group of $\bar{K}$ over $K$. Then we have a commutative diagram

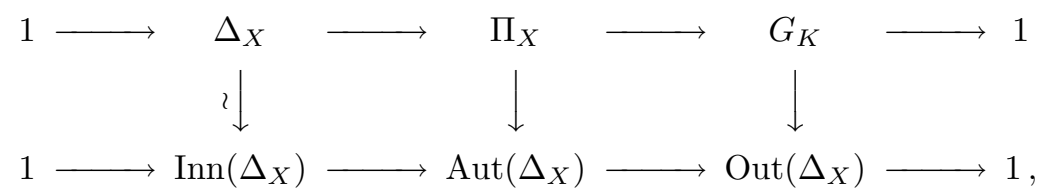

where the horizontal sequences are exact, and the left-hand vertical arrow is an isomorphism; in particular, the right-hand square is cartesian. It follows from [1], Corollary 7 , that $\operatorname{Out}\left(\Delta_{X}\right)$ is almost pro-l, i.e., there exists an open subgroup of $\operatorname{Out}\left(\Delta_{X}\right)$ which is pro- $l$ (where we refer to the discussion entitled "Groups" in Introduction concerning the topology of "Out(-)"). Thus, by replacing $G_{K}$ by an open subgroup of $G_{K}$, assume that the right-hand vertical arrow $G_{K} \rightarrow \operatorname{Out}\left(\Delta_{X}\right)$ in the above diagram factors through a pro-l quotient of $G_{K}$. Then since the right-hand square is cartesian, we conclude that $\Pi_{X}$ is isomorphic to

$$
\left(\Delta_{X} \stackrel{\text { out }}{\rtimes} G_{K}^{(l)}\right) \times G_{K}^{(\neq l)}
$$

where $G_{K}^{(\neq l)}$ is the maximal pro- $(\mathfrak{P r i m e} \backslash\{l\})$ quotient of $G_{K}$; thus, $G_{K}^{(\neq l)}$ is isomorphic to the product of $\mathbb{Z}_{l^{\prime}}$ 's (where $l^{\prime} \in \mathfrak{P r i m e} \backslash\{l\}$ ). Let $L \subseteq \bar{K}$ 
be a finite extension field of $K$ of degree $[L: K]$ prime to $l$ such that $X(K) \neq X(L)$. (In fact, it follows from the "Weil conjecture for curves over finite fields" [cf. e.g., [5], Chapter V, Exercise 1.10] that such an extension field exists.) Let $x \in X(L) \backslash X(K), x^{\prime} \in X(L)$ obtained as the conjugate of $x$ via a generator of the Galois group of the extension $L / K$, and $x_{L} \in X_{L}(L)$ (respectively, $x_{L}^{\prime} \in X_{L}(L)$ ) the $L$-rational point of $X_{L} \stackrel{\text { def }}{=} X \otimes_{K} L$ determined by $x$ (respectively, $\left.x^{\prime}\right)$. Then it follows from the fact $x \notin X(K)$ that $x_{L} \neq x_{L}^{\prime}$; on the other hand, it follows from the fact that $\Pi_{X}$ is isomorphic to $\left(\Delta_{X} \stackrel{\text { out }}{\rtimes} G_{K}^{(l)}\right) \times G_{K}^{(\neq l)}$ that the $\Pi_{X_{L}}$ conjugacy class (where $\Pi_{X_{L}}$ is the geometrically pro-l fundamental group of $X_{L}$ ) of the section of $\Pi_{X_{L}} \rightarrow G_{L}$ corresponding to $x_{L}$ coincides with the $\Pi_{X_{L}}$-conjugacy class of the section of $\Pi_{X_{L}} \rightarrow G_{L}$ corresponding to $x_{L}^{\prime}$. Therefore, $X_{L}$ is not l-separated.

(ii) It follows immediately from the existence of the isomorphism $\Pi_{X} \simeq\left(\Delta_{X} \stackrel{\text { out }}{\rtimes}\right.$ $\left.G_{K}^{(l)}\right) \times G_{K}^{(\neq l)}$ in (i) that there exists an automorphisms of the geometrically pro-l fundamental group $\Pi_{X}$ of a proper hyperbolic curve $X$ which is not Frobenius-preserving.

\section{Acknowledgements}

I would like to thank Professor Shinichi Mochizuki for suggesting the topics, numerous discussions, and many helpful comments. Also, I would like to thank Professor Akio Tamagawa for explaining to me the unpublished result stated in Remark 6, (iii), and the argument in Remark 10, (i). I would also like to thank the referee for useful suggestions and comments. This research was partially supported by JSPS Research Fellowships for Young Scientists.

\section{References}

[1] M. P. Anderson, Exactness properties of profinite completion functors, Topology 13 (1974), 229-239.

[2] M. Asada, On the filtration of topological and pro-l mapping class groups of punctured Riemann surfaces, J. Math. Soc. Japan 48 (1996), no. 1, 13-36.

[3] A. Grothendieck et al., Revêtements étales et groupe fondamental, Lecture Notes in Math., 224, Springer, Berlin, 1971.

[4] Cohomologie locale des faisceaux cohérents et théorèmes de Lefschetz locaux et globaux, North-Holland, Amsterdam, 1968.

[5] R. Hartshorne, Algebraic geometry, Graduate Texts in Mathematics, No. 52, SpringerVerlag, New York-Heidelberg, 1977.

[6] Y. Hoshi, The exactness of the log homotopy sequence, to appear in Hiroshima Math. $\mathrm{J}$. 
[7] Y. Hoshi, On the fundamental groups of log configuration schemes, to appear in Math. J. Okayama Univ.

[8] M. Kaneko, Certain automorphism groups of pro-l fundamental groups of punctured Riemann surfaces, J. Fac. Sci. Univ. Tokyo Sect. IA Math. 36 (1989), no. 2, 363-372.

[9] K. Kato, Logarithmic structures of Fontaine-Illusie, in Algebraic analysis, geometry, and number theory (Baltimore, MD, 1988), 191-224, Johns Hopkins Univ. Press, Baltimore, MD, 1989.

[10] F. F. Knudsen, The projectivity of the moduli space of stable curves. II. The stacks $M_{g, n}$, Math. Scand. 52 (1983), no. 2, 161-199.

[11] W. Magnus, A. Karrass and D. Solitar, Combinatorial group theory: Presentations of groups in terms of generators and relations, Interscience Publishers, New York, 1966.

[12] M. Matsumoto, Galois representations on profinite braid groups on curves, J. Reine Angew. Math. 474 (1996), 169-219.

[13] S. Mochizuki, The absolute anabelian geometry of hyperbolic curves, in Galois theory and modular forms, 77-122, Dev. Math., II, Kluwer Acad. Publ., Boston, MA, 2004

[14] Galois sections in absolute anabelian geometry, Nagoya Math. J. 179 (2005), $17-45$.

[15] _ A combinatorial version of the Grothendieck conjecture, Tohoku Math. J. (2) 59 (2007), no. 3, 455-479.

[16] Absolute anabelian cuspidalizations of proper hyperbolic curves, J. Math. Kyoto Univ. 47 (2007), no. 3, 451-539.

[17] S. Mochizuki and A. Tamagawa, The algebraic and anabelian geometry of configuration spaces, Hokkaido Math. J. 37 (2008), no. 1, 75-131.

[18] D. Mumford, Abelian varieties, Tata Institute of Fundamental Research Studies in Mathematics, No. 5 Published for the Tata Institute of Fundamental Research, Bombay; Oxford University Press, London, 1970.

[19] H. Nakamura, Galois rigidity of the étale fundamental groups of punctured projective lines, J. Reine Angew. Math. 411 (1990), 205-216.

[20] Galois rigidity of pure sphere braid groups and profinite calculus, J. Math. Sci. Univ. Tokyo 1 (1994), no. 1, 71-136.

[21] H. Nakamura, N. Takao and R. Ueno, Some stability properties of Teichmüller modular function fields with pro-l weight structures, Math. Ann. 302 (1995), no. 2, 197-213.

[22] J. Stix, Projective anabelian curves in positive characteristic and descent theory for log-étale covers, Dissertation, Rheinische Friedrich-Wilhelms-Universität Bonn, Bonn, 2002. Bonner Mathematische Schriften, 354. Universität Bonn, Mathematisches Institut, Bonn, 2002.

[23] , A monodromy criterion for extending curves, Int. Math. Res. Not. 2005, no. 29, $1787-1802$.

[24] A. Tamagawa, The Grothendieck conjecture for affine curves, Compositio Math. 109 (1997), no. 2, 135-194. 